\title{
Search for dark matter particles produced in association with a Higgs boson in proton-proton collisions at $\sqrt{\mathrm{s}}=13 \mathrm{TeV}$
}

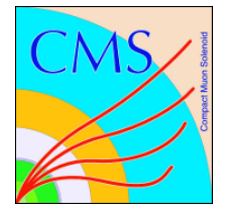

\section{The CMS collaboration}

E-mail: cms-publication-committee-chair@cern.ch

ABSTRACT: A search for dark matter (DM) particles is performed using events with a Higgs boson candidate and large missing transverse momentum. The analysis is based on protonproton collision data at a center-of-mass energy of $13 \mathrm{TeV}$ collected by the CMS experiment at the LHC in 2016, corresponding to an integrated luminosity of $35.9 \mathrm{fb}^{-1}$. The search is performed in five Higgs boson decay channels: $\mathrm{h} \rightarrow \mathrm{b} \overline{\mathrm{b}}, \gamma \gamma, \tau^{+} \tau^{-}, \mathrm{W}^{+} \mathrm{W}^{-}$, and $\mathrm{ZZ}$. The results from the individual channels are combined to maximize the sensitivity of the analysis. No significant excess over the expected standard model background is observed in any of the five channels or in their combination. Limits are set on DM production in the context of two simplified models. The results are also interpreted in terms of a spin-independent DM-nucleon scattering cross section and compared to those from directdetection DM experiments. This is the first search for DM particles produced in association with a Higgs boson decaying to a pair of $\mathrm{W}$ or $\mathrm{Z}$ bosons, and the first statistical combination based on five Higgs boson decay channels.

KEYWORDs: Dark matter, Hadron-Hadron scattering (experiments), Higgs physics

ARXIV EPRINT: 1908.01713 


\section{Contents}

1 Introduction 1

2 The CMS detector and data set 5

$\begin{array}{llr}3 & \text { Signal and background simulation } & 6\end{array}$

$\begin{array}{lll}4 & \text { Event reconstruction } & 7\end{array}$

5 Analysis strategy $\quad 9$

5.1 The $\mathrm{h}(\rightarrow \mathrm{bb})+p_{\mathrm{T}}^{\text {miss }}$ channel $\quad 9$

$\begin{array}{ll}5.2 \text { The } \mathrm{h}(\rightarrow \gamma \gamma)+p_{\mathrm{T}}^{\text {miss }} \text { channel } & 10\end{array}$

$\begin{array}{lll}5.3 & \text { The } \mathrm{h}(\rightarrow \tau \tau)+p_{\mathrm{T}}^{\text {miss }} \text { channel } & 11\end{array}$

5.4 The $\mathrm{h}(\rightarrow \mathrm{WW})+p_{\mathrm{T}}^{\text {miss }}$ channel 11

$\begin{array}{ll}\text { 5.4.1 Background estimation } & 12\end{array}$

5.5 The $\mathrm{h}(\rightarrow \mathrm{ZZ})+p_{\mathrm{T}}^{\text {miss }}$ channel 14

$\begin{array}{lll}\text { 5.5.1 Background estimation } & 15\end{array}$

$\begin{array}{lll}6 & \text { Statistical combination of the search channels } & 17\end{array}$

7 Systematic uncertainties $\quad 18$

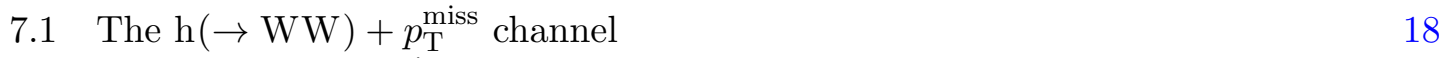

$\begin{array}{lll}7.2 & \text { The } \mathrm{h}(\rightarrow \mathrm{ZZ})+p_{\mathrm{T}}^{\text {miss }} \text { channel } & 19\end{array}$

$\begin{array}{lll}7.3 & \text { Systematic uncertainties in the combination } & 20\end{array}$

8 Results 22

8.1 The $\mathrm{h}(\rightarrow \mathrm{WW})+p_{\mathrm{T}}^{\text {miss }}$ and $\mathrm{h}(\rightarrow \mathrm{ZZ})+p_{\mathrm{T}}^{\text {miss }}$ channels 22

8.2 Results of the statistical combination 23

9 Summary 26

$\begin{array}{ll}\text { The CMS collaboration } & 37\end{array}$

\section{Introduction}

A host of astrophysical and cosmological observations confirm [1-4] that dark matter (DM) exists and makes up $26.4 \%$ of the total energy density of the universe [5]. However, all of the existing evidence for DM is based only on its gravitational interaction. Whether DM interacts with standard model (SM) particles in any other way remains an open question. There are a number of beyond-the-SM theories suggesting a particle nature of DM [6]. Several types of particle candidates for DM are proposed in these models, all compatible 
with the observed relic density of DM in the universe [7]. A favored hypothesis is that the bulk of DM is in the form of stable, electrically neutral, weakly interacting massive particles (WIMPs) [8], with masses in a range between a few $\mathrm{GeV}$ and a few $\mathrm{TeV}$, thus opening the possibility of DM production at high-energy colliders [9].

Traditionally, searches for DM at colliders involve a pair of WIMPs that recoil against a visible SM particle or a set of SM particles. Because of the lack of electric charge and the small interaction cross section, WIMPs do not leave a directly detectable signal, but in a hadron collider experiment their presence can be inferred via an imbalance in the total momentum in the plane transverse to the colliding beams $\left(\vec{p}_{\mathrm{T}}^{\text {miss }}\right)$, as reconstructed in the detector. This scenario gives rise to a potential signature where a set of SM particles, $\mathrm{X}$, are produced recoiling against the DM particles, represented by the $\vec{p}_{\mathrm{T}}^{\text {miss }}$ (the "mono-X" signature). Recent searches at the CERN LHC considered X to be a hadronic jet $[10,11]$, heavy-flavor quarks (bottom and top) $[12,13]$, a photon $[14,15]$, or a $\mathrm{W}$ or $\mathrm{Z}$ boson [11, 16-18].

The discovery of an SM-like Higgs boson [19-21] extended the possibility of probing DM at colliders, complementing other mono-X searches. In this paper we designate the state observed at $125 \mathrm{GeV}$ by the symbol $\mathrm{h}$, since in the context of the theoretical models considered below, it does not correspond to the SM Higgs boson. Here, we present a search for the pair production of DM particles in association with a Higgs boson resulting in the final state $\mathrm{h}+p_{\mathrm{T}}^{\text {miss }}[22,23]$, referred to as the "mono-Higgs". While in a typical mono-X search, the $\mathrm{X}$ particle is emitted as initial-state radiation, this process is strongly suppressed for the case of the Higgs boson because of the smallness of both the Higgs boson Yukawa couplings to light quarks and its loop-suppressed coupling to gluons. Thus, the mono-Higgs production can be either a result of final-state radiation of DM particles, or of a beyond-the-SM interaction of DM particles with the Higgs boson, typically via a mediator particle. A number of searches have been carried out by the ATLAS and CMS collaborations looking for the mono-Higgs signature in several Higgs boson decay channels, at center-of-mass energies of 8 and $13 \mathrm{TeV}$ [24-32]. So far, none of these searches has observed a significant excess of events over the SM expectations.

In this paper, we describe the first search for mono-Higgs production in the $\mathrm{W}^{+} \mathrm{W}^{-}$ and ZZ Higgs boson decay channels, as well as the combination of these searches with the previously published results in the $\mathrm{b} \overline{\mathrm{b}}[30,31], \gamma \gamma$ [32], and $\tau^{+} \tau^{-}[32]$ channels. (Hereafter, for simplicity we refer to $\mathrm{b} \overline{\mathrm{b}}, \tau^{+} \tau^{-}$and $\mathrm{W}^{+} \mathrm{W}^{-}$as $\mathrm{bb}, \tau \tau$ and $\mathrm{WW}$, respectively.) All the analyses are based on a data sample of proton-proton (pp) collisions at $\sqrt{s}=13 \mathrm{TeV}$ collected in 2016 and corresponding to an integrated luminosity of $35.9 \mathrm{fb}^{-1}$.

Two simplified models of DM production recommended by the ATLAS-CMS Dark Matter Forum [33] are investigated. Figure 1 shows representative tree-level Feynman diagrams corresponding to these two models. The diagram on the left describes a type-II two Higgs doublet model (2HDM) $[34,35]$ further extended by a $\mathrm{U}(1)_{\mathrm{Z}^{\prime}}$ group and referred to as the $\mathrm{Z}^{\prime}-2 \mathrm{HDM}$ [36]. In this model, the $\mathrm{Z}^{\prime}$ boson is produced via a quark-antiquark interaction and then decays into a Higgs boson and a pseudoscalar mediator A, which in turn can decay to a pair of Dirac fermion DM particles $\chi$. The diagram on the right shows the production mechanism in the baryonic $\mathrm{Z}^{\prime}$ model [22], where $\mathrm{Z}^{\prime}$ is a vector boson 

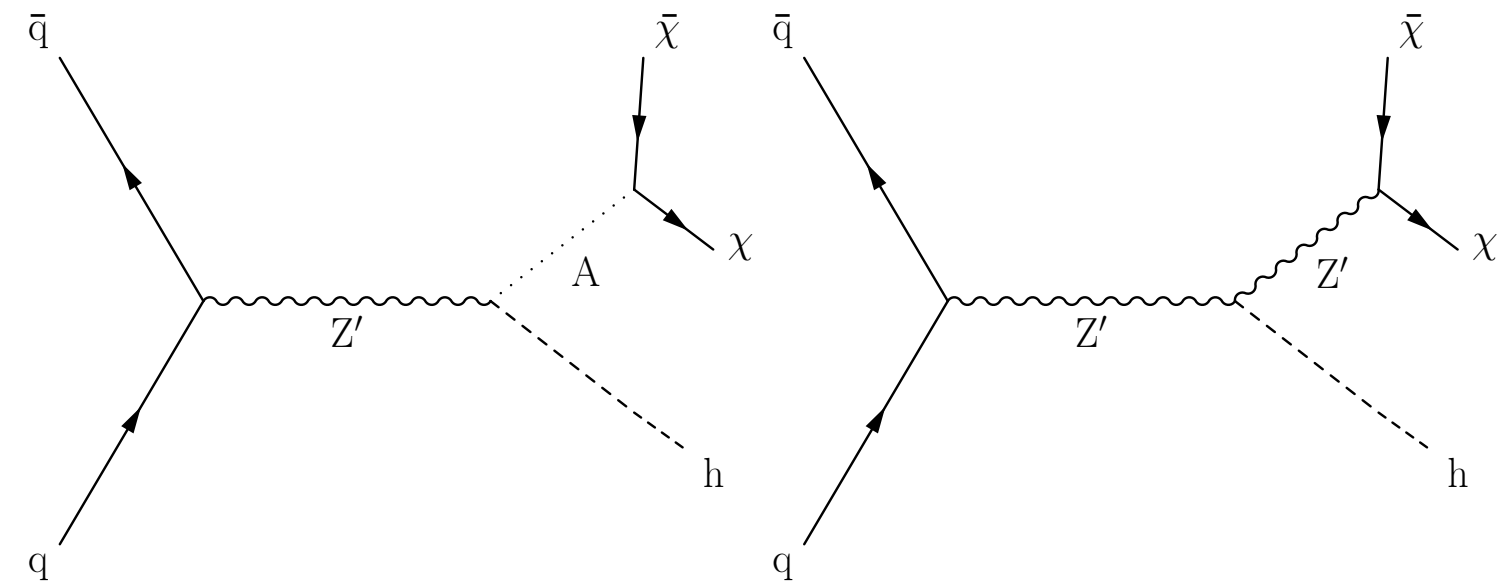

Figure 1. Representative Feynman diagrams for the two benchmark signal models considered in this paper: the $\mathrm{Z}^{\prime}-2 \mathrm{HDM}$ (left) and the baryonic $\mathrm{Z}^{\prime}$ model (right).

corresponding to a new baryon number $\mathrm{U}(1)_{\mathrm{B}}$ symmetry. The $\mathrm{Z}^{\prime}$ boson acts as a $\mathrm{DM}$ mediator and can radiate a Higgs boson before decaying to a pair of DM particles. A baryonic Higgs boson $\mathrm{h}_{\mathrm{b}}$ is introduced to spontaneously break the new symmetry and to generate the $\mathrm{Z}^{\prime}$ boson mass via a coupling that is dependent on the $\mathrm{h}_{\mathrm{b}}$ vacuum expectation value. The $\mathrm{Z}^{\prime}$ boson couplings to quarks and the DM particles are proportional to the $\mathrm{U}(1)_{\mathrm{B}}$ gauge couplings. A mixing between the $\mathrm{h}_{\mathrm{b}}$ and $\mathrm{h}$ states allows the $\mathrm{Z}^{\prime}$ boson to radiate $\mathrm{h}$, resulting in a mono-Higgs signature.

In the $\mathrm{Z}^{\prime}-2 \mathrm{HDM}$, the predicted DM production cross section depends on number of parameters. However, if the mediator A is produced on-shell, the kinematic distributions of the final-state particles depend only on the $\mathrm{Z}^{\prime}$ and $\mathrm{A}$ boson masses, $m_{\mathrm{Z}^{\prime}}$ and $m_{\mathrm{A}}$. In this paper, a scan in $m_{\mathrm{z}^{\prime}}$ between 450 and $4000 \mathrm{GeV}$ and in $m_{\mathrm{A}}$ between 300 and $1000 \mathrm{GeV}$ is performed. The values of $m_{\mathrm{A}}$ below $300 \mathrm{GeV}$ have been already excluded by the existing constraints on flavor changing neutral currents in the $\mathrm{b} \rightarrow \mathrm{s} \gamma$ transitions [34], and hence are not considered in the analysis. The masses of the 2HDM heavy Higgs boson and the charged Higgs boson are both fixed to the $m_{\mathrm{A}}$ mass. The ratio of the vacuum expectation values of the two Higgs doublets, $\tan \beta$, is varied from 0.4 to 10 . The DM particle mass is fixed to $100 \mathrm{GeV}$, the A-DM coupling strength $g_{\chi}$ is fixed to 1 , and the $\mathrm{Z}^{\prime}$ coupling strength to quarks $g_{\mathrm{Z}^{\prime}}$ is fixed to 0.8 . The branching fraction of the decay of A to DM particles $\mathcal{B}(\mathrm{A} \rightarrow \chi \bar{\chi})$ decreases as the mass of the DM candidate $\left(m_{\chi}\right)$ increases, for the range of $m_{\mathrm{A}}$ considered in this analysis. However, since the relative decrease in $\mathcal{B}(\mathrm{A} \rightarrow \chi \bar{\chi})$ is less than $7 \%$ as $m_{\chi}$ increases from 1 to $100 \mathrm{GeV}$, the results shown in this paper for $m_{\chi}=100 \mathrm{GeV}$ are also applicable to lighter DM particles.

The results are expressed in terms of the product of the signal production cross section and branching fraction $\mathcal{B}(\mathrm{A} \rightarrow \chi \bar{\chi})$, where $\mathcal{B}(\mathrm{A} \rightarrow \chi \bar{\chi})$ is $\approx 100 \%$ for $m_{\mathrm{A}}=300 \mathrm{GeV}$ and decreases for $m_{\mathrm{A}}$ greater than twice the mass of the top quark, where the competing decay $\mathrm{A} \rightarrow \mathrm{t} \overline{\mathrm{t}}$ becomes kinematically accessible. The contribution to the mono-Higgs signal from another process possible in the model, $\mathrm{Z}^{\prime} \rightarrow \mathrm{Z}(\rightarrow v \bar{v})+\mathrm{h}$, is not considered in this analysis. Further details on the choice of the model parameters are given in refs. [27, 37]. 

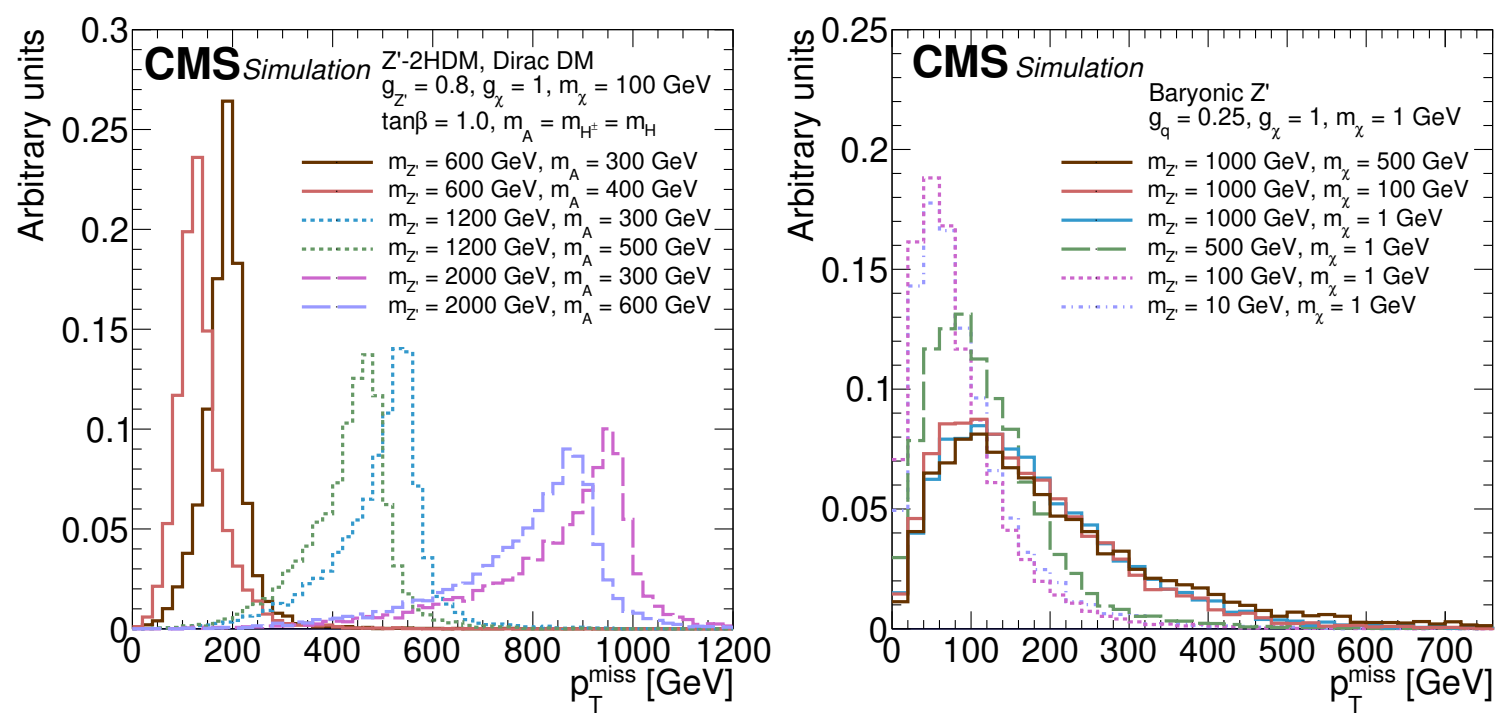

Figure 2. The distribution of $p_{\mathrm{T}}^{\text {miss }}$ at the generator level for the $\mathrm{Z}^{\prime}-2 \mathrm{HDM}$ (left), showing the dependence on the two main model parameters varied in the analysis, $m_{\mathrm{Z}^{\prime}}$ and $m_{\mathrm{A}}$, and for the baryonic $\mathrm{Z}^{\prime}$ model (right), showing the variation of $p_{\mathrm{T}}^{\mathrm{miss}}$ as a function of $m_{\mathrm{z}^{\prime}}$ and $m_{\chi}$. All other parameters of the models are fixed to the values specified in the text. The distributions are normalized to unit area.

We note that for the chosen set of parameters, the values of $m_{\mathrm{z}^{\prime}}$ within our sensitivity reach have been recently excluded by the ATLAS and CMS searches for dijet resonances at $\sqrt{s}=13 \mathrm{TeV}$ [38-41]. Nevertheless, we keep this benchmark, specifically developed for the LHC Run-2 searches [33], to allow a direct comparison with the results of other mono-Higgs searches. Given that the kinematic distributions of the final states depend only very weakly on the value of the $g_{\mathrm{Z}^{\prime}}$ coupling, our results can be reinterpreted for lower $g_{\mathrm{Z}^{\prime}}$ values, where the interplay between the mono-Higgs and the dijet analysis sensitivities changes.

For the baryonic $\mathrm{Z}^{\prime}$ model, $m_{\mathrm{Z}^{\prime}}$ between 100 and $2500 \mathrm{GeV}$ and $m_{\chi}$ between 1 and $700 \mathrm{GeV}$ are used for this study. The $\mathrm{Z}^{\prime}-\mathrm{DM}$ coupling is fixed to $g_{\chi}=1$ and the $\mathrm{Z}^{\prime}$-quark coupling is fixed to $g_{\mathrm{q}}=0.25$. The mixing angle between the baryonic Higgs boson and the SM-like Higgs boson is set to $\sin \theta=0.3$, and the coupling between the $\mathrm{Z}^{\prime}$ boson and $\mathrm{h}$ is assumed to be proportional to $m_{\mathrm{z}^{\prime}}$. The branching fractions of the Higgs boson decays are altered for $m_{\mathrm{Z}^{\prime}} \lesssim m_{\mathrm{h}} / 2$, because the decay $\mathrm{h} \rightarrow \mathrm{Z}^{\prime} \mathrm{Z}^{\prime(*)}$ becomes kinematically accessible. Therefore the region $m_{\mathrm{Z}^{\prime}}<100 \mathrm{GeV}$, for which the modification of the $\mathrm{h}$ branching fractions is sizable, is not considered in the analysis. For both benchmark models, $\mathrm{h}$ is assumed to have a mass of $125 \mathrm{GeV}$. A considerable amount of $p_{\mathrm{T}}^{\text {miss }}$ is expected, as shown in figure 2 . The reason that the $p_{\mathrm{T}}^{\text {miss }}$ spectrum is harder for the $\mathrm{Z}^{\prime}-2 \mathrm{HDM}$ is that the DM particles are produced via a resonant mechanism in this case, whereas for the baryonic $\mathrm{Z}^{\prime}$ model they are not. The difference in shape becomes more marked as $m_{\mathrm{z}^{\prime}}$ increases. In figure 2 (right) it can be seen that the shape of the $p_{\mathrm{T}}^{\text {miss }}$ distribution is almost independent of $m_{\chi}$ in the baryonic $\mathrm{Z}^{\prime}$ model, and depends most strongly on $m_{\mathrm{Z}^{\prime}}$.

Although the signal sensitivity in the $\mathrm{h} \rightarrow$ bb channel is higher than in the other final states considered ( $\gamma \gamma, \tau \tau, \mathrm{WW}$, and $\mathrm{ZZ})$ because of the channel's large branching fraction 
and manageable background in the large- $p_{\mathrm{T}}^{\text {miss }}$ region, the statistical combination of all five decay modes is performed to improve the overall sensitivity. The $\mathrm{h} \rightarrow \gamma \gamma$ and $\mathrm{h} \rightarrow \mathrm{ZZ}$ channels exhibit better resolution in the reconstructed Higgs boson invariant mass, while the $\mathrm{h} \rightarrow \tau \tau, \mathrm{h} \rightarrow \mathrm{WW}$, and $\mathrm{h} \rightarrow \mathrm{ZZ}$ channels benefit from lower SM backgrounds, which results in a higher sensitivity for signals with a soft $p_{\mathrm{T}}^{\text {miss }}$ spectrum.

In the $\mathrm{h} \rightarrow \mathrm{bb}$ channel analysis, the $\mathrm{h}$ is reconstructed from two overlapping $\mathrm{b}$ jets. Thus different approaches are used for the two models, because of the difference in the average Lorentz boost of the Higgs boson, which is higher in the $\mathrm{Z}^{\prime}-2 \mathrm{HDM}$ than in the baryonic $\mathrm{Z}^{\prime}$ model. The Higgs boson is reconstructed using a jet clustering algorithm with a distance parameter of 0.8 for the $\mathrm{Z}^{\prime}-2 \mathrm{HDM}$ and 1.5 for the baryonic $\mathrm{Z}^{\prime}$ model. For the baryonic $\mathrm{Z}^{\prime}$ model, a simultaneous fit of the distribution of the recoil variable in the signal region $(\mathrm{SR})$ and the control regions $(\mathrm{CRs})$ is performed to extract the signal. For the $\mathrm{Z}^{\prime}-2 \mathrm{HDM}$, a parametric fit of the $\mathrm{Z}^{\prime}$ boson transverse mass is used to estimate the major backgrounds and to extract the signal.

The search in the $\mathrm{h} \rightarrow \gamma \gamma$ channel [32] uses a fit to the diphoton invariant mass distribution to extract the signal. This analysis is performed in two categories distinguished by the $p_{\mathrm{T}}^{\text {miss }}$ value, high $(>130 \mathrm{GeV})$ and low $(50-130 \mathrm{GeV})$, in order to be sensitive to a large variety of possible signals.

The search in the $\mathrm{h} \rightarrow \tau \tau$ channel [32] is based on the combination of the events for the three $\tau$ lepton decay modes with the highest branching fractions: $\tau_{\mathrm{h}} \tau_{\mathrm{h}}, \mu \tau_{\mathrm{h}}$, and $\mathrm{e} \tau_{\mathrm{h}}$, where $\tau_{\mathrm{h}}$ denotes a hadronically decaying $\tau$ lepton. After requiring a $p_{\mathrm{T}}^{\text {miss }}(>105 \mathrm{GeV})$ in order to suppress the background sufficiently, the signal is extracted by performing a simultaneous fit in the SR and in the CRs to the transverse mass of the Higgs boson reconstructed from the two $\tau$ leptons. In the $\mathrm{h} \rightarrow \mathrm{WW}$ channel search, the fully leptonic decays of the two $\mathrm{W}$ bosons are considered, requiring one lepton to be an electron and the other to be a muon, in order to reduce the contamination from the $\mathrm{Z} \rightarrow \mathrm{e}^{+} \mathrm{e}^{-}$and $\mathrm{Z} \rightarrow \mu^{+} \mu^{-}$backgrounds. The $\mathrm{h} \rightarrow \mathrm{ZZ}$ search is performed in the fully leptonic decay channel of the $\mathrm{Z}$ boson pair: $\mathrm{h} \rightarrow \mathrm{ZZ} \rightarrow 4 \ell$. The analysis strategy follows closely the measurement of the Higgs boson properties in the same channel [42].

The paper is organized as follows. After a brief introduction of the CMS detector in section 2, the data and simulated event samples are described in section 3 . The event reconstruction and the analysis strategy for each Higgs boson decay mode used in the statistical combination are detailed in sections 4 and 5, respectively. The combination procedure and the main systematic uncertainties are described in sections 6 and 7 , respectively. The results are presented in section 8 , and the paper is summarized in section 9 .

\section{The CMS detector and data set}

The central feature of the CMS apparatus is a superconducting solenoid of $6 \mathrm{~m}$ internal diameter, providing a magnetic field of $3.8 \mathrm{~T}$. Within the solenoid volume are a silicon pixel and strip tracker, a lead tungstate crystal electromagnetic calorimeter (ECAL), and a brass and scintillator hadron calorimeter (HCAL), each composed of a barrel and two endcap sections. Forward calorimeters, made of steel and quartz fibres, extend the pseudorapidity 
$(\eta)$ coverage provided by the barrel and endcap detectors. Muons are detected in gasionization chambers embedded in the steel flux-return yoke outside the solenoid.

Events of interest are selected using a two-tiered trigger system [43]. The first level, composed of custom hardware processors, uses information from the calorimeters and muon detectors to select events at a rate of around $100 \mathrm{kHz}$ in a time of less than $4 \mu \mathrm{s}$. The second level, known as the high-level trigger, consists of a farm of processors running a version of the full event reconstruction software optimized for fast processing, and reduces the event rate to around $1 \mathrm{kHz}$ before data storage.

A more detailed description of the CMS detector, together with a definition of the coordinate system used and the relevant kinematic variables, can be found in ref. [44].

The pp collision data were collected at $\sqrt{s}=13 \mathrm{TeV}$ in 2016. The time spacing between adjacent bunches of $25 \mathrm{~ns}$ leads to an average number of pp interactions per bunch crossing of 23 assuming the pp inelastic cross section of $69.2 \mathrm{mb}$ [45]. The integrated luminosity of the data sample used in all the analyses described in this paper corresponds to $35.9 \mathrm{fb}^{-1}$, after imposing data quality requirements.

\section{Signal and background simulation}

Signal samples for the five Higgs boson decay modes are generated at leading order (LO) in perturbative quantum chromodynamics (QCD) using the MADGRAPH5_aMC@NLO v2.3.0 generator [46, 47], for both the $\mathrm{Z}^{\prime}-2 \mathrm{HDM}$ and baryonic $\mathrm{Z}^{\prime}$ model [33]. The Higgs boson is treated as a stable particle during the generation, and its decays are described subsequently using PYTHIA 8.212 [48].

A detailed description of the simulated samples used for the $\mathrm{h} \rightarrow \mathrm{bb}, \mathrm{h} \rightarrow \gamma \gamma$, and $\mathrm{h} \rightarrow \tau \tau$ analyses can be found in refs. [30-32]. The production of a Higgs boson in association with a $\mathrm{Z}$ boson decaying to a pair of neutrinos is an irreducible background for all the final states considered. Other Higgs boson backgrounds originating from gluongluon fusion ( $\mathrm{ggF}$ ) and vector boson fusion (VBF) production modes are small. These backgrounds are simulated at next-to-LO (NLO) in QCD with POWHEG v2 [49-51].

The main nonresonant backgrounds in the $\mathrm{h} \rightarrow \mathrm{WW}$ analysis are from the continuum WW, single top quark, and top quark pair production. The continuum WW production is simulated in different ways: POWHEG [52] is used to generate q $\bar{q} \rightarrow \mathrm{WW}$ events at NLO precision, whereas gg $\rightarrow \mathrm{WW}$ events are generated at LO using MCFM v7.0 [53-55]. The simulated $\mathrm{q} \overline{\mathrm{q}} \rightarrow \mathrm{WW}$ events are reweighted to reproduce the $p_{\mathrm{T}}^{\mathrm{WW}}$ distribution from the $p_{\mathrm{T}}$-resummed calculation at next-to-NLO (NNLO) plus next-to-next-to-leading logarithmic precision $[56,57]$. The $\mathrm{LO} g \mathrm{~g} \rightarrow \mathrm{WW}$ cross section, obtained directly from MCFM, is further

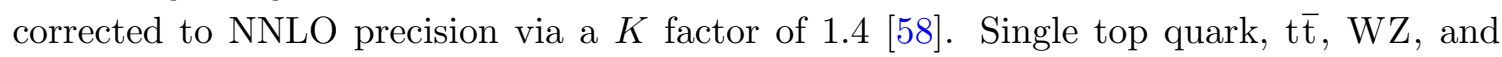
$\mathrm{W} \gamma^{*}$ backgrounds are generated at NLO with POWHEG. Drell-Yan (DY) production of $\mathrm{Z} / \gamma^{*}$ is generated at NLO using MADGRAPH5_aMC@NLO, and the $p_{\mathrm{T}}$ spectrum of the dilepton pairs is reweighted to match the distribution observed in dimuon events in data. Other multiboson processes, such as $\mathrm{W} \gamma, \mathrm{ZZ}$, and $\mathrm{VVV}(\mathrm{V}=\mathrm{W}$ or $\mathrm{Z})$, are generated at NLO with MADGRAPH5_amC@NLO. All samples are normalized to the latest available theoretical cross sections, NLO or higher $[53,54,59]$. 
In the $\mathrm{h} \rightarrow \mathrm{ZZ}$ analysis, the SM production mechanism constitutes a major background because this has the same experimental signature and satisfies the low $p_{\mathrm{T}}^{\text {miss }}$ threshold used in the analysis. It is simulated with POWHEG $[49,50,60]$ in four main production modes: ggF, including quark mass effects [61]; VBF [62]; associated production with a top quark pair ( $\mathrm{t} \overline{\mathrm{th}}$ ) [63]; and associated production with a vector boson (Wh, $\mathrm{Zh}$ ), using the MINLO HVJ [64] extension of POWHEG. In all cases, the Higgs boson is forced to decay via the $\mathrm{h} \rightarrow \mathrm{ZZ} \rightarrow 4 \ell(\ell=\mathrm{e}, \mu$, or $\tau)$ channel. The description of the decay of the Higgs boson to four leptons is obtained using the JHUGEN 7.0.2 generator $[65,66]$. In the case of $\mathrm{Zh}$ and $t \overline{\mathrm{t}} \mathrm{h}$ production, the Higgs boson is allowed to decay as $\mathrm{h} \rightarrow \mathrm{ZZ} \rightarrow 2 \ell+\mathrm{X}$, such that four-lepton events where two leptons originate from the decay of the associated $\mathrm{Z}$ boson or top quarks are also taken into account in the simulation. The cross sections for the processes involving SM Higgs boson production are taken from ref. [67].

All processes are generated using the NNPDF3.0 [68] parton distribution functions (PDFs), with the precision matching the parton-level generator precision. The PYTHIA generator with the underlying event tune CUETP8M1 [69] is used to describe parton showering and fragmentation. The detector response is simulated using a detailed description of the CMS apparatus, based on the GEANT4 package [70]. Additional simulated pp minimum bias interactions in the same or adjacent bunch crossings (pileup) are added to the hard scattering event, with the multiplicity distribution adjusted to match that observed in data.

\section{Event reconstruction}

The particle-flow (PF) algorithm [71] aims to reconstruct and identify each individual particle in an event, with an optimized combination of information from the various elements of the CMS detector. The energy of photons is obtained from the ECAL measurement. The energy of electrons is determined from a combination of the electron momentum at the primary interaction vertex as determined by the tracker, the energy of the corresponding ECAL cluster, and the energy sum of all bremsstrahlung photons spatially compatible with originating from the electron track [72]. The energy of muons is obtained from the curvature of the corresponding track. The energy of charged hadrons is determined from a combination of their momentum measured in the tracker and the matching ECAL and HCAL energy deposits, corrected for zero-suppression effects and for the response function of the calorimeters to hadronic showers. Finally, the energy of neutral hadrons is obtained from the corresponding corrected ECAL and HCAL energies.

Electron candidates are required to have $|\eta|<2.5$. Additional requirements are applied to reject electrons originating from photon conversions in the tracker material or jets misreconstructed as electrons. Electron identification criteria rely on observables sensitive to the bremsstrahlung along the electron trajectory and on the geometrical and momentumenergy matching between the electron track and the associated energy cluster in the ECAL, as well as on the ECAL shower shape observables and association with the primary vertex.

Muon candidates are reconstructed within $|\eta|<2.4$ by combining information from the silicon tracker and the muon system. Identification criteria based on the number of measurements in the tracker and in the muon system, the fit quality of the muon track, and 
its consistency with its origin from the primary vertex are imposed on the muon candidates to reduce the misidentification rate.

For each event, hadronic jets are clustered from PF candidates using the infrared- and collinear-safe anti- $k_{\mathrm{T}}$ algorithm [73, 74], with a distance parameter of 0.4 (AK4 jets) or 0.8 (AK8 jets). Jet momentum is determined as the vectorial sum of all particle momenta in the jet, and is found from simulation to be, on average, within 5 to $10 \%$ of the true momentum over the entire $p_{\mathrm{T}}$ spectrum and detector acceptance. Pileup interactions can result in additional spurious contributions to the jet momentum measurement from tracks and calorimetric energy depositions. To mitigate this effect, tracks identified to be originating from pileup vertices are discarded and a correction based on the jet area [75] is applied to account for the neutral pileup particle contributions. Jet energy corrections are derived from simulation to bring the measured response of jets to that of particle-level jets on average. In situ measurements of the momentum balance in dijet, photon+jet, Z+jet, and multijet events are used to account for any residual differences in the jet energy scale (JES) between data and simulation [76]. The jet energy resolution (JER) amounts typically to $15 \%$ at $p_{\mathrm{T}}=10 \mathrm{GeV}, 8 \%$ at $100 \mathrm{GeV}$, and $4 \%$ at $1 \mathrm{TeV}$. Additional selection criteria are applied to remove jets potentially dominated by anomalous contributions from various subdetector components or reconstruction failures [77].

At large Lorentz boosts, the two b quarks from the Higgs boson decay may produce jets that overlap and make their individual reconstruction difficult. In this case, either the AK8 jets or larger-area jets clustered from PF candidates using the Cambridge-Aachen algorithm [78, 79] with a distance parameter of 1.5 (CA15 jets) are used. To reduce the impact of particles arising from pileup interactions when reconstructing AK8 or CA15 jets, the four-vector of each PF candidate matched to the jet is scaled with a weight calculated with the pileup-per-particle identification algorithm [80] prior to the clustering. The CA15 jets are also required to be central $(|\eta|<2.4)$. The "soft-drop" jet grooming algorithm [81] is applied to remove soft, large-angle radiation from the jets. The mass of a groomed AK8 or CA15 jet are referred to as $m_{\mathrm{SD}}$.

To identify jets originating from $\mathrm{b}$ quark fragmentation (b jets), two b tagging algorithms are used. The combined secondary vertex (CSVv2) [82] and the combined multivariate analysis (cMVAv2) algorithms [82] are used to identify AK4 jets originating from b quarks by their characteristic displaced vertices. For the AK8 jets, subjets inside the jet are required to be tagged as b jets using the CSVv2 algorithm. A likelihood for the CA15 jet to contain two b quarks is derived by combining the information from the primary and secondary vertices and tracks in a multivariate discriminant optimized to distinguish CA15 jets originating from the $\mathrm{h} \rightarrow$ bb decay from those produced by energetic light-flavor quarks or gluons [31].

Hadronically decaying $\tau$ leptons are reconstructed from jets using the hadrons-plusstrips algorithm [83]. This algorithm uses combinations of reconstructed charged hadrons and energy deposits in the ECAL to identify the three most common hadronic $\tau$ lepton decay modes: 1-prong, 1-prong $+\pi^{0}(\mathrm{~s})$, and 3-prong. The $\tau_{\mathrm{h}}$ candidates are further required to satisfy the isolation criteria with an efficiency of $65(50) \%$ and a misidentification probability of $0.8(0.2) \%$ in the $\tau_{\mathrm{h}} \tau_{\mathrm{h}}\left(\mathrm{e} \tau_{\mathrm{h}}\right.$ or $\left.\mu \tau_{\mathrm{h}}\right)$ channel. 


\begin{tabular}{|lcc|}
\hline Decay channel & Final state or category & Reference \\
\hline \multicolumn{1}{|c}{$\mathrm{h} \rightarrow \mathrm{bb}$} & AK8 jet $\left(\mathrm{Z}^{\prime}-2 \mathrm{HDM}\right)$ & {$[30]$} \\
& CA15 jet $\left(\right.$ Baryonic $\left.\mathrm{Z}^{\prime}\right)$ & {$[31]$} \\
$\mathrm{h} \rightarrow \gamma \gamma$ & $p_{\mathrm{T}}^{\text {miss }} \in 50-130 \mathrm{GeV}$ & {$[32]$} \\
& $p_{\mathrm{T}}^{\text {miss }}>130 \mathrm{GeV}$ & {$[32]$} \\
$\mathrm{h} \rightarrow \tau \tau$ & $\tau_{\mathrm{h}} \tau_{\mathrm{h}}$ & {$[32]$} \\
& $\mu \tau_{\mathrm{h}}$ & {$[32]$} \\
$\mathrm{h} \rightarrow \mathrm{WW}$ & $\mathrm{e} \tau_{\mathrm{h}}$ & {$[32]$} \\
& $\mathrm{e} v \mu \nu$ & - \\
$\mathrm{h} \rightarrow \mathrm{ZZ}$ & $4 \mathrm{e}$ & - \\
& $4 \mu$ & - \\
\hline
\end{tabular}

Table 1. Summary of the individual channels entering the combination. Analyses are categorized based on the model, $p_{\mathrm{T}}^{\text {miss }}$ selection, and subsequent decay products listed here. The categorization is the same for both the $\mathrm{Z}^{\prime}-2 \mathrm{HDM}$ and the Baryonic $\mathrm{Z}^{\prime}$ model for all decay channels except, as indicated, $\mathrm{h} \rightarrow \mathrm{bb}$. A dash ("-") in the last column implies that the analysis is presented in this paper.

The $\vec{p}_{\mathrm{T}}^{\text {miss }}$ is reconstructed as the negative vectorial sum of all PF particle candidate momenta projected on the plane transverse to the beams. Since the presence of pileup induces a degradation of the $p_{\mathrm{T}}^{\text {miss }}$ measurement $\left(p_{\mathrm{T}}^{\text {miss }}\right.$ resolution varies almost linearly from 15 to $30 \%$ as the number of vertices increases from 5 to 30 [84]), affecting mostly backgrounds with no genuine $p_{\mathrm{T}}^{\text {miss }}$, an alternative definition of $p_{\mathrm{T}}^{\text {miss }}$ that is constructed only using the charged PF candidates ("tracker $p_{\mathrm{T}}^{\text {miss") }}$ ) is used in the $\mathrm{h} \rightarrow \mathrm{WW}$ analysis. In the rest of the paper, $p_{\mathrm{T}}^{\text {miss }}$ corresponds to the $\mathrm{PF} p_{\mathrm{T}}^{\text {miss }}$, unless specified otherwise.

\section{Analysis strategy}

In this section we briefly discuss the analysis strategies in the previously published [30-32] $\mathrm{h} \rightarrow \mathrm{bb}, \mathrm{h} \rightarrow \gamma \gamma$, and $\mathrm{h} \rightarrow \tau \tau$, channels, and provide full descriptions of the new analyses in the $\mathrm{h} \rightarrow \mathrm{WW}$ and $\mathrm{h} \rightarrow \mathrm{ZZ}$ decay channels. The summary of all the decay channels contributing to the combination is presented in table 1 .

\subsection{The $\mathrm{h}(\rightarrow \mathrm{bb})+p_{\mathrm{T}}^{\text {miss }}$ channel}

The events used in this final state are selected using a triggers that require large amount $(>90$ or $>120 \mathrm{GeV})$ of $p_{\mathrm{T}}^{\text {miss }}$, or $H_{\mathrm{T}}^{\text {miss }}$ defined as the magnitude of the vectorial sum of the transverse momenta of all jets with $p_{\mathrm{T}}>20 \mathrm{GeV}$ in an event. The trigger selection is $96(100) \%$ efficient for events that subsequently have $p_{\mathrm{T}}^{\text {miss }}>200(350) \mathrm{GeV}$ in the offline reconstruction. As can be seen in figure 2, the Lorentz boosts of the Higgs boson are different for the $\mathrm{Z}^{\prime}-2 \mathrm{HDM}$ and baryonic $\mathrm{Z}^{\prime}$ model. The events with large boost in the 
$\mathrm{Z}^{\prime}-2 \mathrm{HDM}$ are reconstructed using a large-radius AK8 jet with $p_{\mathrm{T}}>200 \mathrm{GeV}$ and $|\eta|<2.4$. In addition, the $\mathrm{h} \rightarrow \mathrm{bb}$ topology is selected by requiring at least one subjet of the AK8 jet to be $\mathrm{b}$ tagged. The analysis considers separately two categories, distinguished by the number of $\mathrm{b}$ tagged subjets in the event, one or two, the latter being the high-purity category with higher sensitivity. For events with lower boost in the baryonic $\mathrm{Z}^{\prime}$ model, Higgs boson candidates are reconstructed using CA15 jets.

To select the $\mathrm{h} \rightarrow \mathrm{bb}$ candidates using the AK8 jet, one or both subjets are required to pass the loose $\mathrm{b}$ tagging criteria, which has an efficiency of $85 \%$, and a misidentification rate of about $10 \%$ for jets originating from light-flavor quarks or gluons. In the case of the CA15 jets, a multivariate double b tagging algorithm [82] is used to discriminate the signal from the background of light-flavor jets [31], with an efficiency of $50 \%$ and a misidentification rate of $10 \%$. The AK8 (CA15) analysis requires the Higgs boson candidate mass to be in the 105-135 (100-150) GeV range to reduce nonresonant backgrounds. The difference in the two mass window requirements is primarily driven by the differences in the performance of the two algorithms and in the jet mass resolutions. For both analyses, the mass window was chosen to maximize the signal sensitivity. In order to further reduce the background contributions from $\mathrm{W}+$ jets and $t \overline{\mathrm{t}}$ production, events with an electron, muon, photon $\left(p_{\mathrm{T}}>10 \mathrm{GeV}\right)$, or $\tau_{\mathrm{h}}\left(p_{\mathrm{T}}>18 \mathrm{GeV}\right)$ candidates passing loose identification and isolation criteria are vetoed. Furthermore, in the AK8 analysis, the number of additional b tagged AK4 jets with $p_{\mathrm{T}}>20 \mathrm{GeV}$ is required to be zero, while in the CA15 analysis, the number of AK4 jets with $p_{\mathrm{T}}>30 \mathrm{GeV}$, well-separated from the CA15 jet in the event, is required to be at most one. The sensitivity of the analyses is further enhanced by using jet substructure variables. The full details of the event selection for the AK8 and CA15 jet analyses can be found in refs. [30] and [31], respectively.

\subsection{The $\mathrm{h}(\rightarrow \gamma \gamma)+p_{\mathrm{T}}^{\text {miss }}$ channel}

Signal candidate events in the $\mathrm{h} \rightarrow \gamma \gamma$ analysis are selected using a diphoton trigger with asymmetric $p_{\mathrm{T}}$ thresholds of 30 and $18 \mathrm{GeV}$ on the leading and subleading photons, respectively, and loose identification and isolation requirements imposed on both photon candidates. The diphoton invariant mass is further required to exceed $90 \mathrm{GeV}$.

Slightly higher thresholds of $30(20) \mathrm{GeV}$ on the leading (subleading) photon $p_{\mathrm{T}}$ and of $95 \mathrm{GeV}$ on the diphoton mass are used offline. The photon candidates are required to pass the isolation criteria if the spatial distance in $\eta-\phi$ plane $\left(\Delta R=\sqrt{(\Delta \eta)^{2}+(\Delta \phi)^{2}}\right)$ between the two photons exceeds 0.3 . The isolation selection is not used for photons that are coming from the decay of a highly Lorentz-boosted Higgs boson, as the two photons are likely to be found in the isolation cone of one another. The analysis is performed in two categories distinguished by the value of $p_{\mathrm{T}}^{\text {miss }}$ : high- $p_{\mathrm{T}}^{\text {miss }}(>130 \mathrm{GeV})$ and low- $p_{\mathrm{T}}^{\text {miss }}$ $(50-130 \mathrm{GeV})$.

The multijet background, with a large $p_{\mathrm{T}}^{\text {miss }}$ in an event originating from the mismeasurement of the energy of one or more jets, is reduced by allowing at most two jets with $p_{\mathrm{T}}>30 \mathrm{GeV}$. To suppress the contribution from the multijet background, the azimuthal separation between the direction of any jet with $p_{\mathrm{T}}>50 \mathrm{GeV}$ and $\vec{p}_{\mathrm{T}}^{\text {miss }}$ is required to exceed 0.5 radians. Finally, to select signal-like events with the DM particles recoiling 
against the Higgs boson, the azimuthal separation between $\vec{p}_{\mathrm{T}}^{\text {miss }}$ and the direction of the Higgs boson candidate reconstructed from the diphoton system is required to exceed 2.1 radians. More details of the event selection can be found in ref. [32].

\subsection{The $\mathbf{h}(\rightarrow \tau \tau)+p_{\mathrm{T}}^{\text {miss }}$ channel}

In the $\mathrm{h} \rightarrow \tau \tau$ analysis, the three final states with the highest branching fractions are analyzed: $\tau_{\mathrm{h}} \tau_{\mathrm{h}}, \mu \tau_{\mathrm{h}}$, and $\mathrm{e} \tau_{\mathrm{h}}$. The events are selected online with a trigger requiring the presence of two isolated $\tau_{\mathrm{h}}$ candidates in the $\tau_{\mathrm{h}} \tau_{\mathrm{h}}$ final state, and a single-muon (singleelectron) trigger in the $\mu \tau_{\mathrm{h}}\left(\mathrm{e} \tau_{\mathrm{h}}\right)$ final state. Electron, muon, and $\tau_{\mathrm{h}}$ candidates passing the identification and isolation criteria are combined to reconstruct a Higgs boson candidate in these three final states. The signal events are then selected with the requirements: $p_{\mathrm{T}}^{\text {miss }}>105 \mathrm{GeV}$ and visible $p_{\mathrm{T}}$ of the $\tau \tau$ system $>65 \mathrm{GeV}$. To ensure that the $\tau \tau$ system originates from the Higgs boson, the visible mass of the $\tau \tau$ system is required to be less than $125 \mathrm{GeV}$. In order to reduce the contribution from multilepton and $\mathrm{t} \overline{\mathrm{t}}$ backgrounds, the events are vetoed if an additional electron, muon, or a b tagged jet is present. More details of the event selection can be found in ref. [32].

\subsection{The $\mathrm{h}(\rightarrow \mathrm{WW})+p_{\mathrm{T}}^{\text {miss }}$ channel}

The search in the $\mathrm{h} \rightarrow \mathrm{WW}$ decay channel is performed in the fully leptonic, opposite-sign, different-flavor $(\mathrm{e} \mu)$ final state, which has relatively low backgrounds. The presence of the neutrinos and the DM particles escaping detection results in large $p_{\mathrm{T}}^{\text {miss }}$ in signal events. The selected $\mathrm{e} \mu+p_{\mathrm{T}}^{\text {miss }}$ events include a contribution from the $\mathrm{h} \rightarrow \mathrm{WW} \rightarrow \tau \tau \nu_{\tau} \nu_{\tau}$ process with both $\tau$ leptons decaying leptonically. Several background processes can lead to the same final state, dominated by $\mathrm{t} \overline{\mathrm{t}}$ and $\mathrm{WW}$ production.

Online, events are selected using a suite of single- and double-lepton triggers. In the offline selection, the leading (subleading) lepton is required to have $p_{\mathrm{T}}>25(20) \mathrm{GeV}$. Electron and muon candidates are required to be well-identified and isolated to reject the background from leptons inside jets. Backgrounds from low-mass resonances are reduced by requiring the dilepton invariant mass $\left(m_{\ell \ell}\right)$ to exceed $12 \mathrm{GeV}$, while backgrounds with three leptons in the final state are reduced by vetoing events with an additional well-identified lepton with $p_{\mathrm{T}}>10 \mathrm{GeV}$. The $p_{\mathrm{T}}^{\text {miss }}$ in the event is required to exceed $20 \mathrm{GeV}$ in order to reduce the contribution from instrumental backgrounds and $\mathrm{Z} / \gamma^{*} \rightarrow \tau^{+} \tau^{-}$decays. To suppress the latter background, the $p_{\mathrm{T}}$ of the dilepton system is required to be greater than $30 \mathrm{GeV}$ and the transverse mass of the dilepton and $\vec{p}_{\mathrm{T}}^{\text {miss }}$ system, $m_{\mathrm{T}}^{\mathrm{h}}$, is required to be greater than $40 \mathrm{GeV}$. In order to reduce the $\mathrm{Z} / \gamma^{*} \rightarrow \mathrm{e}^{+} \mathrm{e}^{-}, \mu^{+} \mu^{-}$or $\tau^{+} \tau^{-}$background with $p_{\mathrm{T}}^{\text {miss }}$ originating either from $\tau$ lepton decays or from mismeasurement of the energies of e, $\mu$ or additional jets, a variable $p_{\mathrm{T}, \text { proj }}^{\text {miss }}[85]$ is introduced. This is defined as the projection of $\vec{p}_{\mathrm{T}}^{\text {miss }}$ in the plane transverse to the direction of the nearest lepton, unless this lepton is situated in the opposite hemisphere to $\vec{p}_{\mathrm{T}}^{\text {miss }}$, in which case $p_{\mathrm{T} \text {,proj }}^{\text {miss }}$ is taken to be $p_{\mathrm{T}}^{\text {miss }}$ itself. A selection using this variable efficiently rejects $\mathrm{Z} / \gamma^{*} \rightarrow \ell \ell$ background events, in which the $\vec{p}_{\mathrm{T}}^{\text {miss }}$ is preferentially aligned with leptons. Since the $p_{\mathrm{T}}^{\text {miss }}$ resolution is degraded by pileup, a quantity $p_{\mathrm{T}, \mathrm{mp}}^{\text {miss }}$ is defined as the smaller of the two $p_{\mathrm{T}, \text { proj }}^{\text {miss }}$ values: the one based on all the $\mathrm{PF}$ candidates in the event, and the one based only on the reconstructed tracks originating 

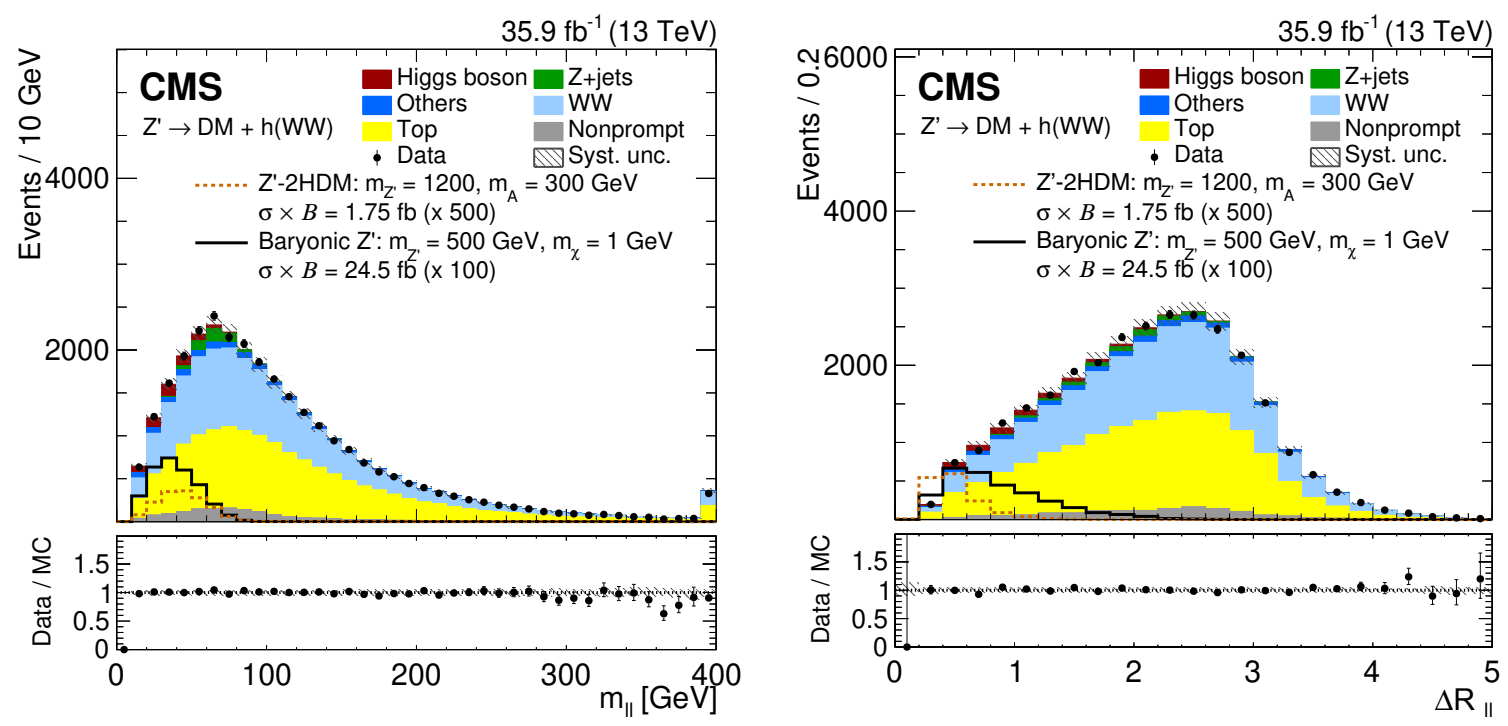

Figure 3. The distribution of $m_{\ell \ell}$ (left) and $\Delta R_{\ell \ell}$ (right) after the preselection, expected from MC simulation (stacked histograms) and observed in data (points with vertical bars). The systematic uncertainties, discussed in section 7.1, are shown by the hatched region. Two signal benchmarks, corresponding to the $\mathrm{Z}^{\prime}-2 \mathrm{HDM}$ (dotted orange line) and baryonic $\mathrm{Z}^{\prime}$ (solid black line) model are superimposed. The signal is normalized to the product of cross section and $\mathcal{B}$, where $\mathcal{B}$ represents the $\mathrm{h} \rightarrow \mathrm{WW}$ branching fraction. The signal distributions are scaled up by a factor 500 (100) for the $\mathrm{Z}^{\prime}-2 \mathrm{HDM}$ (baryonic $\mathrm{Z}^{\prime}$ model), to make them more visible. The lower panel shows the ratio of the data to the predicted SM background.

from the primary vertex. A requirement $p_{\mathrm{T}, \mathrm{mp}}^{\text {miss }}>20 \mathrm{GeV}$ is effective in suppressing the targeted background. The above requirements define the event preselection.

The expected signal significance is enhanced by introducing two additional selections: $m_{\ell \ell}<76 \mathrm{GeV}$ and the distance in $\eta-\phi$ space between the two leptons $\Delta R_{\ell \ell}<2.5$, as illustrated in figure 3. The first requirement exploits the fact that the invariant mass of the leptons coming from the $\mathrm{h} \rightarrow \mathrm{WW}$ decay tends to be low because of the presence of the two neutrinos in the decay chain and of the scalar nature of the Higgs boson. The second requirement utilizes the fact that the Higgs boson in signal events recoils against the DM particles and is highly boosted.

\subsubsection{Background estimation}

Since full kinematic reconstruction of the Higgs boson mass and $p_{\mathrm{T}}$ is impossible in this decay channel because of the presence of undetected neutrinos and DM particles, to maximize the sensitivity of the search, a boosted decision tree (BDT) multivariate classifier has been trained for each of the two signal models. The BDT exploits the following input variables:

- transverse masses: $m_{\mathrm{T}}^{\mathrm{h}}, m_{\mathrm{T}}^{\mathrm{W}_{1}}, m_{\mathrm{T}}^{\mathrm{W}_{2}}$;

- lepton transverse momenta: $p_{\mathrm{T}}^{\ell \ell}, p_{\mathrm{T}}^{\ell_{1}}, p_{\mathrm{T}}^{\ell_{2}}$; 
- missing transverse momenta: $\mathrm{PF} p_{\mathrm{T}}^{\text {miss }}$, tracker $p_{\mathrm{T}}^{\text {miss }}, p_{\mathrm{T}, \mathrm{mp}}^{\mathrm{miss}}$;

- angular variables: $\Delta R_{\ell \ell}, \Delta \phi_{\ell \ell}, \Delta \phi_{p_{\mathrm{T}}^{\text {miss }} \ell_{1}}, \Delta \phi_{p_{\mathrm{T}}^{\text {miss }} \ell_{2}} ;$ and

- dilepton invariant mass: $m_{\ell \ell}$.

Here, $m_{\mathrm{T}}^{\mathrm{W}_{i}}=\sqrt{2 p_{\mathrm{T}}^{\ell_{i}} p_{\mathrm{T}}^{\text {miss }}\left(1-\cos \Delta \phi_{p_{\mathrm{T}}^{\text {miss }} \ell_{i}}\right)}$, where $i=1(i=2)$ defines the transverse mass of $\vec{p}_{\mathrm{T}}^{\text {miss }}$ and the leading (subleading) lepton in the event, and $\Delta \phi_{\ell \ell}$ is the azimuthal angle between the directions of the two lepton momenta.

For both benchmark models, the BDT training considers processes with two prompt leptons and genuine $p_{\mathrm{T}}^{\text {miss }}$ (WW, $\mathrm{t} \overline{\mathrm{t}}, \mathrm{tW}$, and $\mathrm{h} \rightarrow \mathrm{WW}$ production) as the backgrounds. For the $\mathrm{Z}^{\prime}-2 \mathrm{HDM}$ (baryonic $\mathrm{Z}^{\prime}$ ) model, simulated signal samples with $m_{\mathrm{A}}=300 \mathrm{GeV}$ $\left(m_{\chi}=1 \mathrm{GeV}\right)$ with various values of $m_{\mathrm{Z}^{\prime}}$ have been used for training. The chosen signal points correspond to the region of maximum sensitivity of the $h \rightarrow W W$ analysis for both models.

The main background processes arise from top quark ( $t \overline{\mathrm{t}}$ and single top quark production, mainly tW), nonresonant WW events, and nonprompt leptons. The contribution of nonprompt-lepton background in the SR is determined entirely from data, while the contributions of the top quark, WW, and $\mathrm{Z} / \gamma^{*} \rightarrow \tau^{+} \tau^{-}$background are estimated using simulated samples. The normalizations of simulated backgrounds are obtained using dedicated CRs that are included in the maximum-likelihood fit used to extract the signal, together with the SR. Smaller backgrounds, WZ and $\mathrm{W}^{*}$, are estimated using simulation after applying a normalization factor estimated in the respective CRs. The WZ CR is defined by requiring the presence of two opposite-sign, same-flavor leptons, compatible with the decay of a $\mathrm{Z}$ boson and one additional lepton of a different flavor, consistent with originating from a $\mathrm{W}$ boson decay. In the $\mathrm{W} \gamma^{*} \mathrm{CR}$, the two leptons produced by the decay of the virtual photon are required to have $p_{\mathrm{T}}>8 \mathrm{GeV}$ and be isolated. Since the two leptons may be close to each other, the isolation is computed without taking into account the contribution of lepton tracks falling in the isolation cone. An additional lepton consistent with originating from the $\mathrm{W}$ decay is required. The $\mathrm{WZ}$ and $\mathrm{W} \gamma^{*}$ CRs are not used in the maximum-likelihood fit; instead, the normalization scale factors are extracted and directly applied to the corresponding simulated samples. The remaining backgrounds from diboson and triboson production are estimated directly from simulation.

The $\mathrm{gg} \rightarrow \mathrm{W}^{+} \mathrm{W}^{-}$and $\mathrm{qq} \rightarrow \mathrm{W}^{+} \mathrm{W}^{-}$backgrounds are estimated from simulation normalized as discussed in section 3. The main feature of these processes is that, as the two $\mathrm{W}$ bosons do not originate in a decay of the Higgs boson, their invariant mass does not peak at the Higgs boson mass. For this reason, events in the corresponding CR are required to have a large dilepton invariant mass, achieved by inverting the $\mathrm{SR} m_{\ell \ell}<76 \mathrm{GeV}$ requirement.

The estimation of the top quark background is performed in two steps. First, a top quark enriched $\mathrm{CR}$ is defined to measure a scale factor quantifying the difference in the $\mathrm{b}$ tagging efficiencies and mistag rates in data and simulation. This CR is obtained from the SR selection by inverting the $\mathrm{b}$ tagged jet veto. In second step, the scale factor is 
applied to the corresponding simulated samples with a weight per event that depends on the number, flavor, and kinematic distributions of jets.

The $\mathrm{W}+$ jets production contributes as a background in the $\mathrm{h} \rightarrow \mathrm{WW}$ analysis when a jet is misidentified as a lepton. A CR is defined to contain events with one isolated lepton and another lepton candidate that fails the nominal isolation criteria, but passes a looser selection. The probability for a jet satisfying this looser selection to pass the nominal one is estimated from data in an independent sample dominated by nonprompt leptons from multijet production. This probability is parameterized as a function of the $p_{\mathrm{T}}$ and $\eta$ of the lepton and applied to the events in the CR. In order to estimate the nonprompt lepton contamination in the SR, a validation region enriched in nonprompt leptons is defined with the same requirement as the SR, but requiring same-sign e $\mu$ pairs. The maximum discrepancy between data and prediction in the validation region, amounting to $\approx 30 \%$, is taken as the uncertainty in the $\mathrm{W}+$ jets background prediction.

The $\mathrm{Z} / \gamma^{*} \rightarrow \tau^{+} \tau^{-}$background is estimated from simulation, after reweighting the $\mathrm{Z}$ boson $p_{\mathrm{T}}$ spectrum to match the distribution measured in data. The normalization of the simulated sample is estimated from data using events in the $m_{\mathrm{T}}^{\mathrm{h}}<40 \mathrm{GeV}$ region. A normalization factor is then extracted from this region and applied to the SR.

The main difference between the present analysis and the measurement of the SM Higgs boson properties in the same channel [85] is in the signal extraction method. The latter analysis uses a multidimensional fit to the $m_{\mathrm{T}}^{\mathrm{h}}, m_{\ell \ell}$, and $p_{\mathrm{T}}^{\ell_{2}}$ distributions, whereas a fit to the BDT discriminant distribution is used in the present analysis.

\subsection{The $\mathrm{h}(\rightarrow \mathrm{ZZ})+p_{\mathrm{T}}^{\text {miss }}$ channel}

The search in the $\mathrm{h} \rightarrow \mathrm{ZZ}$ channel is performed in all-leptonic final states. Each of the $\mathrm{Z}$ bosons decays to a pair of leptons (electrons or muons, including those coming from leptonic $\tau$ decays) resulting in a four-lepton signature. The main advantages of the $\mathrm{h} \rightarrow \mathrm{ZZ} \rightarrow 4 \ell$ over other Higgs boson decay modes are that the Higgs boson candidates can be fully reconstructed, with an excellent mass resolution, and the backgrounds are easily controlled. On the other hand, this channel suffers from a relatively small branching fraction compared to most of other Higgs boson decay channels. The three different final states (four electrons, four muons, and two electrons and two muons) are analyzed individually and then combined to obtain final results. The selection of the $\mathrm{h} \rightarrow \mathrm{ZZ} \rightarrow 4 \ell$ events follows closely that used in the measurement of the Higgs boson properties in the four-lepton channel, based on the same data set [42].

The signal event topology is defined by the presence of four charged leptons (4e, $4 \mu$, or $2 \mathrm{e} 2 \mu)$ and significant $p_{\mathrm{T}}^{\text {miss }}$ produced by the undetected DM particles. The events are selected online with triggers requiring the presence of two isolated leptons (ee, $\mu \mu$, or $\mathrm{e} \mu$ ), with asymmetric $p_{\mathrm{T}}$ thresholds of 23 (17) GeV on the leading and 12 (8) GeV on the subleading electron (muon). Dilepton triggers account for most of the signal efficiency in all three final states. In order to maximize the signal acceptance, trilepton triggers with lower $p_{\mathrm{T}}$ thresholds and no isolation requirements are added, as well as single-electron and single-muon triggers with isolated lepton $p_{\mathrm{T}}$ thresholds of 27 and $22 \mathrm{GeV}$, respectively [42]. 
The reconstruction and selection of the Higgs boson candidates proceeds first by selecting two $\mathrm{Z}$ boson candidates, defined as pairs of opposite-sign, same-flavor leptons $\left(\mathrm{e}^{+} \mathrm{e}^{-}\right.$, $\mu^{+} \mu^{-}$) passing the selection criteria and satisfying $12<m_{\ell \ell(\gamma)}<120 \mathrm{GeV}$, where the $\mathrm{Z}$ boson candidate mass $m_{\ell \ell(\gamma)}$ includes the contribution of photons identified as coming from final-state radiation [42]. The $\mathrm{ZZ}$ candidates are then defined as pairs of $\mathrm{Z}$ boson candidates not sharing any of the leptons. The $\mathrm{Z}$ candidate with the reconstructed mass closest to the nominal $\mathrm{Z}$ boson mass $[86]$ is denoted as $\mathrm{Z}_{1}$, and the other one is denoted as $\mathrm{Z}_{2}$. All the leptons used to select the $\mathrm{Z}_{1}$ and $\mathrm{Z}_{2}$ candidates must be separated by $\Delta R\left(\ell_{i}, \ell_{j}\right)>0.02$.

The leading (subleading) of the four leptons must have $p_{\mathrm{T}}>20(10) \mathrm{GeV}$, and the $\mathrm{Z}_{1}$ candidate must have a reconstructed mass $m_{\mathrm{Z}_{1}}$ above $40 \mathrm{GeV}$. In the $4 \mathrm{e}$ and $4 \mu$ channels, if an alternative $Z_{i} Z_{j}$ candidate based on the same four leptons is found, the event is discarded if $m_{\mathrm{Z}_{i}}$ is closer to the nominal $\mathrm{Z}$ boson mass than $m_{\mathrm{Z}_{1}}$. This requirement rejects events with an on-shell $\mathrm{Z}$ boson produced in association with a low-mass dilepton resonance. In order to suppress the contribution of QCD production of low-mass dilepton resonances, all four opposite-sign pairs that can be built with the four leptons (regardless of the lepton flavor) must satisfy $m_{\ell_{i} \ell_{j}}>4 \mathrm{GeV}$ and the four-lepton invariant mass must satisfy $m_{4 \ell}>70 \mathrm{GeV}$. If more than one $\mathrm{ZZ}$ candidate passes the selection, the one with the highest value of the scalar $p_{\mathrm{T}}$ sum of four leptons is chosen. The above requirements define the event preselection.

The $m_{4 \ell}$ distribution for selected $\mathrm{ZZ}$ candidates exhibits a peak around $125 \mathrm{GeV}$, as expected for both the SM Higgs boson production and signal. However, because of the much lower cross section, the potential signal is overwhelmed by the background after the SM Higgs boson selection, as shown in figure 4 (left). The distribution of $p_{\mathrm{T}}^{\text {miss }}$ for selected $\mathrm{ZZ}$ candidates is shown in figure 4 (right).

After the preselection, the remaining background comes from the SM Higgs boson (mostly $\mathrm{Vh}$ ), $\mathrm{t} \overline{\mathrm{t}}+\mathrm{V}$, and $\mathrm{VV} / \mathrm{VVV}$ production. Another background dominated by the $\mathrm{Z}+$ jets production ("Z+X") [42] arises from secondary leptons misidentified as prompt because of the decay of heavy-flavor hadrons and light mesons within jets, and, in the case of electrons, from photon conversions or charged hadrons overlapping with photons from $\pi^{0} \rightarrow \gamma \gamma$ decays. The nonprompt-lepton background also contains smaller contributions from $\mathrm{t} \overline{\mathrm{t}}+$ jets, $\mathrm{Z} \gamma+$ jets, $\mathrm{WZ}+$ jets, and $\mathrm{WW}+$ jets events, with a jet misidentified as a prompt lepton. These backgrounds do not exhibit peak in the distribution of $m_{4 \ell}$, and are reduced by applying a selection on the $m_{4 \ell}$ around the Higgs boson mass $\left(115<m_{4 \ell}<\right.$ $135 \mathrm{GeV}$ ), by rejecting events with more than four leptons, and by requiring the number of $\mathrm{b}$ tagged jets in the event to be less than two.

\subsubsection{Background estimation}

The dominant irreducible backgrounds from the SM Higgs boson and nonresonant ZZ production are determined from simulation, while the $\mathrm{Z}+\mathrm{X}$ background is determined from data [42]. All other backgrounds are determined from simulation. Background contributions from the SM Higgs boson production in association with a $\mathrm{Z}$ boson or a $\mathrm{t} \overline{\mathrm{t}}$ pair, followed by the $\mathrm{h} \rightarrow \mathrm{WW} \rightarrow 2 \ell 2 \nu$ decay, have been studied with simulated events and found to be negligible. 

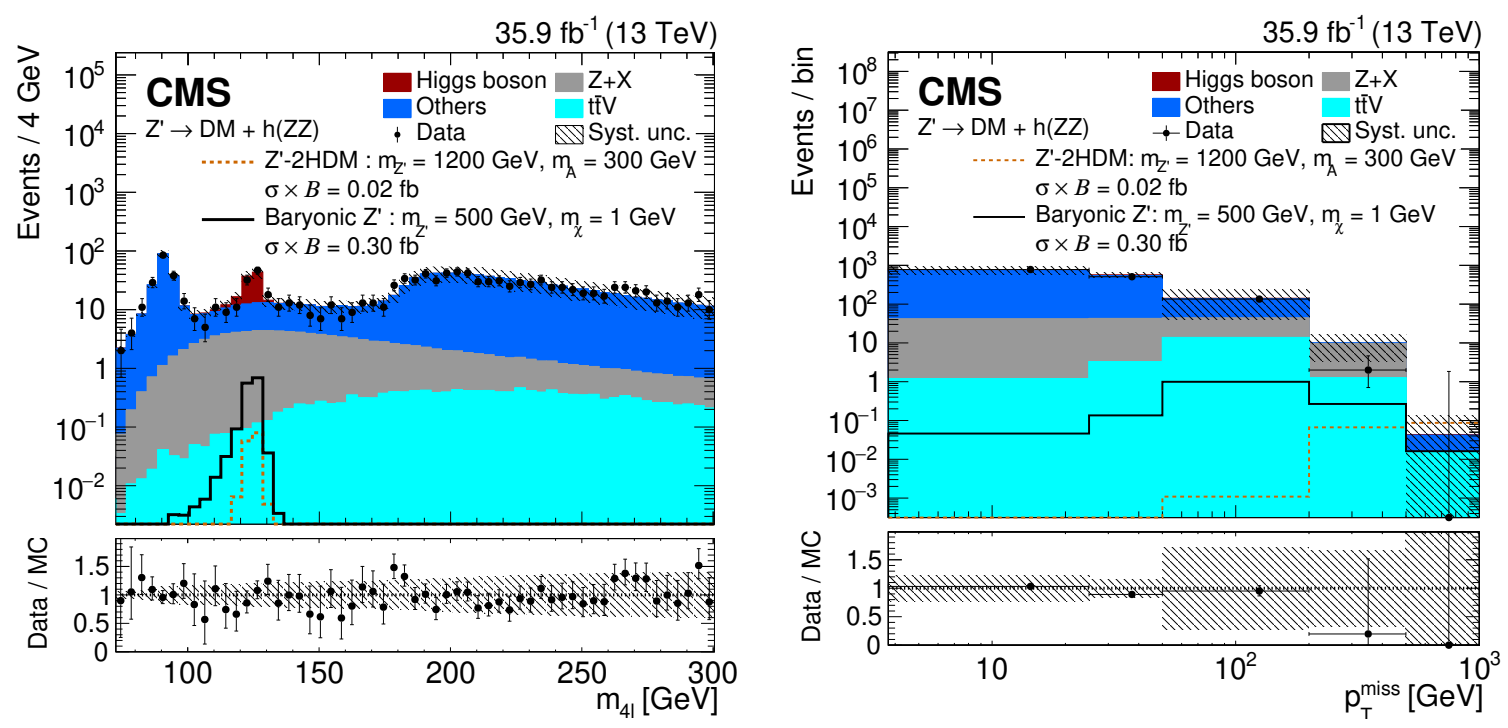

Figure 4. The four-lepton invariant mass (left) and $p_{\mathrm{T}}^{\text {miss }}$ distributions (right) after the preselection, expected from MC simulation (stacked histograms) and observed in data (points with vertical bars). The systematic uncertainties, discussed in section 7.1, are shown by the hatched region. Two signal benchmarks, corresponding to the $\mathrm{Z}^{\prime}-2 \mathrm{HDM}$ (dotted orange line) and baryonic Z' (solid black line) model are superimposed. The signal is normalized to the product of cross section and $\mathcal{B}$, where $\mathcal{B}$ represents the $\mathrm{h} \rightarrow \mathrm{ZZ}$ branching fraction. The lower panel shows the ratio of the data to the predicted SM background.

The $\mathrm{Z}+\mathrm{X}$ background is estimated from data by first determining the lepton misidentification probability in a dedicated CR and then using it to derive the background contribution in the SR. The lepton misidentification probability is defined as the probability that a lepton passing a loose selection with relaxed identification or isolation criteria also passes the tight selection criteria. The misidentification probability is measured in a $\mathrm{Z}+$ lepton $\mathrm{CR}$ where the $\mathrm{Z}$ boson candidate (with the mass within $7 \mathrm{GeV}$ of the nominal $\mathrm{Z}$ boson mass) is formed from the two selected leptons passing the tight identification criteria, and an additional lepton is required to pass the loose selection. This sample is dominated by $\mathrm{Z}+$ nonprompt-lepton events. The electron and muon misidentification probabilities are measured as functions of the lepton candidate $p_{\mathrm{T}}$, its location in the barrel or endcap region of the ECAL or the muon system, and $p_{\mathrm{T}}^{\text {miss }}$ in the event, using $\mathrm{Z}(\rightarrow \ell \ell)+\mathrm{e}$ and $\mathrm{Z}(\rightarrow \ell \ell)+\mu$ events, respectively, in the $\mathrm{Z}+$ lepton $\mathrm{CR}$. The misidentification probabilities are found to be independent of the charge of the lepton within the uncertainties.

The strategy for applying the lepton misidentification probabilities relies on two additional CRs. The first CR is defined by requiring that the two leptons that do not form the $\mathrm{Z}_{1}$ candidate, pass only the loose, but not the tight identification criteria. This $\mathrm{CR}$ defines the "2 pass +2 fail" ( $2 \mathrm{P} 2 \mathrm{~F})$ sample and is expected to be populated by events that intrinsically have only two prompt leptons (mostly from DY production, with a small contribution from $t \bar{t}$ and $\mathrm{Z}_{\gamma}$ events). The second $\mathrm{CR}$ is defined by requiring only one of the four leptons to fail the tight identification and isolation criteria and defines the " 3 pass +1 fail" (3P1F) sample, which is expected to be populated by the type of events 


\begin{tabular}{|lccccc|}
\hline Object & $\mathrm{h} \rightarrow \mathrm{bb}$ & $\mathrm{h} \rightarrow \gamma \gamma$ & $\mathrm{h} \rightarrow \tau \tau$ & $\mathrm{h} \rightarrow \mathrm{WW}$ & $\mathrm{h} \rightarrow \mathrm{ZZ}$ \\
\hline Electron & $=0$ & - & $=0$ & $=0$ & $=0$ \\
Muon & $=0$ & - & $=0$ & $=0$ & $=0$ \\
$\tau$ lepton & $=0$ & - & - & $=0$ & - \\
Photon & $=0$ & - & - & - & - \\
AK4 Jet & $\leq 1$ & $\leq 2$ & - & - & - \\
b tagged AK4 jet & $=0$ & - & $=0$ & $=0$ & $\leq 1$ \\
\hline
\end{tabular}

Table 2. Summary of the maximum number of additional objects allowed in an event for each analysis. A dash means that no restriction on the corresponding object is applied in the corresponding analysis.

that populate the $2 \mathrm{P} 2 \mathrm{~F}$ CR, but with different relative proportions, as well as by $\mathrm{WZ}+$ jets events with three prompt leptons.

\section{Statistical combination of the search channels}

The analyses in the five channels described above are almost completely statistically independent of each other, allowing these analyses to be combined without accounting for the possibility of events being selected in more than one final state. Whenever an explicit veto ensuring the strict mutual exclusivity of the channels is not placed in a particular analysis, it was checked that there are no overlapping events with the other channels. The summary of the vetoes on additional objects, namely electrons, muons, $\tau$ leptons, photons, jets, and $\mathrm{b}$ tagged jets, in each analysis is presented in table 2 . These selections not only reduce the major backgrounds, but also ensure the nearly complete mutual exclusivity of the analyses considered for the combination. The overlap in the SR is zero and for the CR it is less than $0.01 \%$, i.e., it is much smaller than the systematic uncertainty in the analysis.

The combination of the analyses in the five Higgs boson decay channels is performed for both the $\mathrm{Z}^{\prime}-2 \mathrm{HDM}$ and the baryonic $\mathrm{Z}^{\prime}$ model. For each model, the $\mathrm{h} \rightarrow \mathrm{bb}$ channel dominates the sensitivity in most of the phase space, and hence the combined results are dominated by this channel. However, there are regions of the parameter space that are hard to probe with $\mathrm{h} \rightarrow$ bb decays, and other channels play a major role there. The analysis strategies for all channels are the same for both models, except for the $\mathrm{h} \rightarrow \mathrm{bb}$ channel, where two different strategies are used because of the difference in the Lorentz boost of the Higgs boson. In this channel, the results for the $\mathrm{Z}^{\prime}-2 \mathrm{HDM}$ are taken from ref. [30], whereas for the baryonic $\mathrm{Z}^{\prime}$ model, the results from ref. [31] are used in the combination.

For the $\mathrm{Z}^{\prime}-2 \mathrm{HDM}$, the two parameters that we scan are $m_{\mathrm{Z}^{\prime}}$ and $m_{\mathrm{A}}$. All five analyses contribute to the combination in the ranges $800<m_{\mathrm{Z}^{\prime}}<2500 \mathrm{GeV}$ and $300<m_{\mathrm{A}}<$ $800 \mathrm{GeV}$. For $m_{\mathrm{z}^{\prime}}<800 \mathrm{GeV}$, it is not possible to perform the $\mathrm{h} \rightarrow$ bb analysis efficiently, therefore only four other decay channels are used for the combination. For $m_{\mathrm{Z}^{\prime}}>2500 \mathrm{GeV}$ and $m_{\mathrm{A}}>800 \mathrm{GeV}$ the signal selection efficiency is significant only for the $\mathrm{h} \rightarrow$ bb decay mode, hence only the $\mathrm{h} \rightarrow \mathrm{bb}$ channel contributes in this region. 
For the baryonic $\mathrm{Z}^{\prime}$ model, the two parameters that we scan are $m_{\mathrm{Z}^{\prime}}$ and $m_{\chi}$, and all five analyses are performed in the full phase space considered for the combination. Since the maximum sensitivity for all the analyses is achieved for $m_{\chi}=1 \mathrm{GeV}$, the comparison of individual analyses is shown only for this DM particle mass, to demonstrate the improvement in the sensitivity achieved in the combination of individual channels.

\section{Systematic uncertainties}

A number of systematic uncertainties are considered in the combination, broadly divided into two categories: theoretical and experimental. Theoretical uncertainties are considered fully correlated among all five channels. Only the systematic uncertainties attributed to the experimental sources that are correlated between different channels are described for the combined result in section 7.3. The details of all experimental systematic uncertainties in the $\mathrm{h} \rightarrow \mathrm{bb}$ analysis using AK8 jets are described in ref. [30] and those for the analysis using CA15 jets are described in ref. [31]; for the $\mathrm{h} \rightarrow \gamma \gamma$ and $\mathrm{h} \rightarrow \tau \tau$ channels they are given in ref. [32]; and for the $\mathrm{h} \rightarrow \mathrm{WW}$ and $\mathrm{h} \rightarrow \mathrm{ZZ}$ analyses they are discussed in this section.

\subsection{The $\mathrm{h}(\rightarrow \mathrm{WW})+p_{\mathrm{T}}^{\text {miss }}$ channel}

The normalization and the kinematic shapes of the BDT discriminant distributions for the main backgrounds are derived from data CRs, and therefore systematic uncertainties in both the normalization and shapes are considered.

For the nonprompt-lepton background the uncertainty amounts to approximately $30 \%$, and covers the uncertainty in the lepton misidentification rate, the dependence on the CR background composition, and the statistical component because of the finite event count in the CR.

The top quark background CR is included as an additional category in the signal extraction fit. The kinematic shapes of the top quark background are taken from simulation corrected for the $\mathrm{b}$ tagging scale factors, with the uncertainties covering the difference between the b tagging efficiency in data and simulation [82]. A similar procedure is applied for the DY background, by defining a $\mathrm{CR}$ in low- $m_{\mathrm{T}}$ phase space, and to the nonresonant WW background, for which a high- $m_{\ell \ell} \mathrm{CR}$ is defined. The top quark and DY background normalizations are correlated between their respective CRs and the SR and are left unconstrained in the fit. The change in the PDF, the renormalization and the factorization scale variations from their nominal values lead to migration of the top quark and $\mathrm{Z} / \gamma^{*} \rightarrow \tau^{+} \tau^{-}$ background events between the respective CRs and the SR. To take into account this effect, the change in the top quark $\left(\mathrm{Z} / \gamma^{*} \rightarrow \tau^{+} \tau^{-}\right)$background yield is used as an additional 1 (2)\% uncertainty in the corresponding CR. The shapes of the WZ and other minor backgrounds are taken from simulation and normalized to their theoretical predictions, with the theoretical uncertainties estimated. The uncertainties related to the modeling of $p_{\mathrm{T}}^{\mathrm{miss}}$ are estimated by considering the effect of varying the lepton energy scale on $p_{\mathrm{T}}^{\text {miss }}$.

Experimental uncertainties are estimated by applying scale factors between data and simulation, and/or by smearing of certain kinematic variables in simulation, with the cor- 


\begin{tabular}{|lcc|}
\hline Source of uncertainty & Process & Size \\
\hline Trigger efficiency & Simulated samples & $2 \%$ \\
Nonprompt lepton bkg. & Nonprompt lepton bkg. & $30 \%$ \\
$\mathrm{WZ}$ bkg. normalization & $\mathrm{WZ}$ & $16 \%$ \\
$\mathrm{~W} \gamma^{(*)}$ bkg. normalization & $\mathrm{W} \gamma^{(*)}$ & $26 \%$ \\
$\mathrm{~h} \rightarrow \mathrm{WW}$ branching fraction & Signal & $\sim 1 \%$ \\
Single t $/ \mathrm{t} \overline{\mathrm{t}}$ cross section ratio & Top quark & $8 \%$ \\
Top quark $p_{\mathrm{T}}$ & Top quark & $1 \%$ \\
$\mathrm{gg} \rightarrow \mathrm{W} \mathrm{W}^{-}$LO to NNLO $K$ factor & $\mathrm{gg} \rightarrow \mathrm{W}^{+} \mathrm{W}^{-}$ & $15 \%$ \\
$p_{\mathrm{T}}^{\mathrm{WW}}$ resummation & $\mathrm{qq} \rightarrow \mathrm{W}^{+} \mathrm{W}^{-}$ & $\sim 5 \%$ \\
Top quark CR to SR transfer factor & Top quark & $1 \%$ \\
$\mathrm{Z} / \gamma^{*} \rightarrow \tau^{+} \tau^{-}$CR to SR transfer factor & $\mathrm{Z} / \gamma^{*} \rightarrow \tau^{+} \tau^{-}$ & $2 \%$ \\
Simulated sample event count & Simulated samples & $2-70 \%$ \\
\hline
\end{tabular}

Table 3. Systematic uncertainties affecting the $\mathrm{h} \rightarrow \mathrm{WW}$ analysis.

responding changes further propagated to all analysis variables. The signal acceptance uncertainty associated with the combination of single-lepton and dilepton triggers is measured to be $2 \%$. The uncertainty in the ratio between the single top quark and top quark pair production cross sections, $8 \%$ at $13 \mathrm{TeV}$ [87], has been also included, as it affects the top quark background yield from the maximum-likelihood fit used to extract the signal and dominant backgrounds. The uncertainty in the $p_{\mathrm{T}}$ spectrum of the top quark has been applied to all the observables in order to cover the difference between the simulated and observed spectra [88], and is of the order of $1 \%$.

The uncertainty in the Higgs boson branching fraction for the $\mathrm{h} \rightarrow \mathrm{WW}$ decay is about $1 \%$ [67]. The uncertainty in the NNLO $K$ factor applied to the LO gg $\rightarrow$ WW cross section estimate is $15 \%$ [89]. The $p_{\mathrm{T}}^{\mathrm{WW}}$ spectrum in the qq $\rightarrow \mathrm{WW}$ sample has been reweighted to match the resummed calculation $[56,57]$. The associated shape uncertainties related to the missing higher-order corrections are modeled by varying the factorization, renormalization, and resummation scales up and down independently by a factor of 2 from their nominal values [56]. Finally, uncertainties arising from the limited size of the simulated samples are included for each bin of the BDT discriminant distributions, in each category. The main sources of the uncertainties affecting the analysis are listed in table 3 .

\subsection{The $\mathrm{h}(\rightarrow \mathrm{ZZ})+p_{\mathrm{T}}^{\text {miss }}$ channel}

A source of systematic uncertainty in the nonprompt-lepton background estimate potentially arises from the difference in the composition of the SM background processes with nonprompt leptons $\left(\mathrm{Z}_{\gamma}+\right.$ jets, $\mathrm{t} \overline{\mathrm{t}}, \mathrm{Z}_{\gamma}+$ jets $)$ contributing to the CRs where the lepton misidentification rate is measured and applied. This uncertainty can be estimated by measuring the misidentification rates in simulation for the $2 \mathrm{P} 2 \mathrm{~F}$ and $3 \mathrm{P} 1 \mathrm{~F}$ CRs. Half of 
the difference between the misidentification rates obtained from simulation in these two CRs is used as a measure of the systematic uncertainty in the lepton misidentification rate and is further propagated to the uncertainty in the nonprompt-lepton background, and amounts to $43 \%$ for the $4 \mathrm{e}, 36 \%$ for the $4 \mu$, and $40 \%$ for the $2 \mathrm{e} 2 \mu$ final states.

The uncertainty in the full signal selection efficiency is at the level of $1 \%$. The uncertainty in the $m_{4 \ell}$ resolution from the uncertainty in the per-lepton energy resolution is about 20\% [42] and affects the signal and all the backgrounds from Higgs boson production.

In addition, there are two types of systematic uncertainties related to the modeling of $p_{\mathrm{T}}^{\text {miss }}$. The first uncertainty is related to the approximately Gaussian core of the resolution function for correctly measured jets and other physics objects and corresponds to the uncertainty in the genuine $p_{\mathrm{T}}^{\mathrm{miss}}$. The second uncertainty, attributed to significant mismeasurement of $p_{\mathrm{T}}^{\text {miss }}$, is an uncertainty in the "mismeasured" $p_{\mathrm{T}}^{\text {miss }}$.

The uncertainties from the modeling of genuine $p_{\mathrm{T}}^{\text {miss }}$ are measured by varying the parameters associated with the corrections applied to $p_{\mathrm{T}}^{\text {miss }}$ and by propagating those variations to the $p_{\mathrm{T}}^{\text {miss }}$ calculation, after applying the full analysis selection. Each correction is varied up and down by one standard deviation of the input distribution. The corrections used in this calculation come from JES, JER, muon, electron, photon, and the unclustered energy scales.

The uncertainty in the mismeasured $p_{\mathrm{T}}^{\text {miss }}$ is obtained from a sample with significant contributions from misidentified leptons and mismeasured jets, obtained by requiring an opposite-sign, same-flavor dilepton pair passing the $\mathrm{Z}_{1}$ candidate selection, and an additional same-sign, same-flavor pair ("OS+SS" sample). This sample is enriched in misidentified leptons that form the same-sign pair and is expected to lead to significant mismeasurement of $p_{\mathrm{T}}^{\text {miss }}$, not already covered by the uncertainties in the Gaussian core discussed above. We derive the mismeasured $p_{\mathrm{T}}^{\text {miss }}$ uncertainty from the comparison of the $p_{\mathrm{T}}^{\text {miss }}$ shapes in the "OS+SS" sample and in the SR, with a requirement that the $m_{4 \ell}$ be outside the Higgs boson invariant mass peak $\left(\left|m_{4 \ell}-125 \mathrm{GeV}\right|>10 \mathrm{GeV}\right)$. The uncertainty in mismeasured $p_{\mathrm{T}}^{\text {miss }}$ is applied to the $\mathrm{Z}+\mathrm{X}$ sample only, since the effect is expected to be negligible when four genuine leptons are produced, as is the case for the signal and for most of the simulated background samples.

An uncertainty of $10 \%$ in the $K$ factor used for the $g \mathrm{~g} \rightarrow \mathrm{ZZ}$ prediction is applied [89]. A systematic uncertainty of $2 \%$ in the $\mathrm{h} \rightarrow \mathrm{ZZ} \rightarrow 4 \ell$ branching fraction [67] affects both signal and the SM Higgs boson background yields. Theoretical uncertainties in the $t \overline{\mathrm{t}}+\mathrm{V}$ background cross sections are taken from ref. [90]. A summary of the experimental uncertainties is given in table 4 .

\subsection{Systematic uncertainties in the combination}

The uncertainties associated with the background normalization and fit parameters are assumed to be uncorrelated, whereas those associated with the standard object selection are considered fully correlated and are summarized in table 5. In all five decay channels, a normalization uncertainty of $2.5 \%$ for simulated samples is used to account for the uncertainty in the measurement of the integrated luminosity [91]. Also fully correlated across all channels are the systematic uncertainties related to theoretical calculations of the Higgs 


\begin{tabular}{|c|c|c|}
\hline Source of uncertainty & Process & Rate \\
\hline Trigger selection & Simulated samples & $2 \%$ \\
\hline$m_{4 \ell}$ resolution & Higgs boson & $20 \%$ \\
\hline $\mathrm{h} \rightarrow \mathrm{ZZ} \rightarrow 4 \ell$ branching fraction & Higgs boson & $2 \%$ \\
\hline $\mathrm{gg} \rightarrow \mathrm{ZZ} \mathrm{NNLO}$ to LO $K$ factor & gg $\rightarrow$ ZZ bkg. & $10 \%$ \\
\hline Genuine $p_{\mathrm{T}}^{\text {miss }}$ & Simulated samples (Shape) & $7-26 \%$ \\
\hline Mismeasured $p_{\mathrm{T}}^{\text {miss }}$ & Z+X bkg. (Shape) & $2-30 \%$ \\
\hline $\mathrm{Z}+\mathrm{X}$ bkg. yield & Z+X bkg. (Yield) & $36-43 \%$ \\
\hline $\mathrm{t} \overline{\mathrm{t}}+\mathrm{V}$ bkg. yield & $\mathrm{t} \overline{\mathrm{t}}+\mathrm{V}$ bkg. & $27-34 \%$ \\
\hline
\end{tabular}

Table 4. Systematic uncertainties affecting the $\mathrm{h} \rightarrow \mathrm{ZZ}$ analysis.

\begin{tabular}{|c|c|c|c|c|c|c|}
\hline \multirow[t]{2}{*}{ Source } & \multicolumn{2}{|c|}{$\mathrm{h} \rightarrow \mathrm{bb}$} & \multirow[t]{2}{*}{$\mathrm{h} \rightarrow \gamma \gamma$} & \multirow[t]{2}{*}{$\mathrm{h} \rightarrow \tau \tau$} & \multirow[t]{2}{*}{$\mathrm{h} \rightarrow \mathrm{WW}$} & \multirow[t]{2}{*}{$\mathrm{h} \rightarrow \mathrm{ZZ}$} \\
\hline & $\mathrm{Z}^{\prime}-2 \mathrm{HDM}$ & Baryonic $\mathrm{Z}^{\prime}$ & & & & \\
\hline AK4 jet $\mathrm{b}$ tagging & \multirow{2}{*}{$3-11 \%$} & Uncorr. $(3-4 \%)$ & - & $4 \%$ & Shape $(1 \%)$ & $1 \%$ \\
\hline AK4 jet b mistag & & Shape $(5-7 \%)$ & - & $2-5 \%$ & Shape $(1 \%)$ & - \\
\hline e ident. efficiency & $4 \%$ & $2 \%$ & - & $2 \%$ & Shape $(2 \%)$ & $2.5-9.0 \%$ \\
\hline$\mu$ ident. efficiency & $4 \%$ & $2 \%$ & - & $2 \%$ & Shape $(2 \%)$ & $2.5-9.0 \%$ \\
\hline$\tau_{\mathrm{h}}$ ident. efficiency & $3 \%$ & $3 \%$ & - & $4.5 \%$ & Shape $(1 \%)$ & - \\
\hline e energy scale & $1 \%$ & - & - & - & Shape $(1 \%)$ & $3 \%$ \\
\hline$\mu$ energy scale & $1 \%$ & - & - & - & Shape (1\%) & $0.4 \%$ \\
\hline JES & - & Uncorr. (4\%) & - & Shape $(<10 \%)$ & Shape $(3 \%)$ & $2-3 \%$ \\
\hline Int. luminosity & $2.5 \%$ & $2.5 \%$ & $2.5 \%$ & $2.5 \%$ & $2.5 \%$ & $2.5 \%$ \\
\hline Signal (PDF, scales) & $0.3-9.0 \%$ & $0.3-9.0 \%$ & $0.3-9.0 \%$ & $0.3-9.0 \%$ & $0.3-9.0 \%$ & $0.3-9.0 \%$ \\
\hline
\end{tabular}

Table 5. Systematic uncertainties in the combination of channels, along with the type (rate/shape) of uncertainty affecting signal and background processes, correlated amongst at least two final states. For the rate uncertainties, the percentage of the prior value is quoted, while for shape uncertainties an estimate of the impact of systematic uncertainties on the yield is also listed. A dash ("_,") implies that a given uncertainty does not affect the analysis. Whenever an uncertainty is present but kept uncorrelated in a particular channel, this is mentioned explicitly. The effect of the b jet mistag rate uncertainty is very small in the $h \rightarrow b b \mathrm{Z}^{\prime}-2 \mathrm{HDM}$ analysis and hence it is added to the effect of the b tagging efficiency uncertainty in quadrature.

boson production cross section, PDFs, and renormalization and factorization scale uncertainties estimated using the recommendations of the PDF4LHC [92] and LHC Higgs Cross Section [67] working groups, respectively. These uncertainties range from 0.3 to $9.0 \%$.

Uncertainties from imprecise knowledge of the JES are evaluated by propagating the uncertainties in the JES for individual jets in an event, which depend on the jet $p_{\mathrm{T}}$ and $\eta$, to all the analysis quantities. The uncertainties in the selection of $b$ tagged AK4 jets are taken into account using the uncertainties in the b tagging efficiency and misidentification rate estimated from the difference between data and simulation [82]. The uncertainty due to the difference in the performance of electron, muon, and $\tau$ lepton identification between 


\begin{tabular}{|lc|}
\hline Channel & Event yield \\
\hline SM Higgs boson $\left(m_{\mathrm{h}}=125 \mathrm{GeV}\right)$ & $598 \pm 55$ \\
Top quark & $4450 \pm 310$ \\
$\mathrm{WW}$ & $4500 \pm 160$ \\
Other VV $/ \mathrm{VVV}$ & $449 \pm 44$ \\
$\mathrm{Z}+$ jets & $367 \pm 42$ \\
Nonprompt lepton bkg. & $660 \pm 210$ \\
Total bkg. & $11030 \pm 410$ \\
$\mathrm{Z}^{\prime}-2 \mathrm{HDM}\left(m_{\mathrm{Z}^{\prime}}=1200 \mathrm{GeV}, m_{\mathrm{A}}=300 \mathrm{GeV}\right)$ & $3.04 \pm 0.10$ \\
Baryonic $\mathrm{Z}^{\prime}\left(m_{\mathrm{Z}^{\prime}}=500 \mathrm{GeV}, m_{\chi}=1 \mathrm{GeV}\right)$ & $29.60 \pm 0.89$ \\
Observed & 11172 \\
\hline
\end{tabular}

Table 6. The post-fit signal and background event yields, and the observed number of events in data, for the $\mathrm{h} \rightarrow \mathrm{WW}$ analysis. The expected numbers of signal events for the two signal hypotheses are also reported, one for each benchmark model. The total uncertainty, including both statistical and systematic components, is quoted for the expected signal and backgrounds yields.

data and simulation is taken into account for individual decay channels and considered fully correlated in the statistical combination. An uncertainty of $1-3 \%$ in the electron energy scale and an uncertainty of $0.4-1.0 \%$ in the muon energy scale are considered to be correlated in the combination.

\section{Results}

The event selection described in section 5 has been used to discriminate the mono-Higgs signal from backgrounds in each channel. The observed yields in data and the expected event yields for the signal and background processes in the $h \rightarrow b b, h \rightarrow \gamma \gamma$, and $h \rightarrow \tau \tau$ channels can be found in refs. [30-32]. The corresponding yields for the $\mathrm{h} \rightarrow \mathrm{WW}$ and $\mathrm{h} \rightarrow \mathrm{ZZ}$ analyses are discussed in section 8.1. Table 6, 7 and figures 5, 6 show one signal mass hypothesis for each model, normalized to the respective cross section. For the $\mathrm{Z}^{\prime}$ $2 \mathrm{HDM}$, the signal is normalized to the cross section calculated for mass values of $\mathrm{Z}^{\prime}$ and $\mathrm{A}$ bosons of 1200 and $300 \mathrm{GeV}$, respectively, and for $g_{\mathrm{Z}^{\prime}}=0.8, \tan \beta=1$. For the baryonic $\mathrm{Z}^{\prime}$ model, the signal is normalized to the cross section corresponding to the $\mathrm{Z}^{\prime}$ and $m_{\chi}$ masses of 500 and $1000 \mathrm{GeV}$, respectively, and for $g_{\chi}=1, g_{\mathrm{q}}=0.25$.

\subsection{The $\mathrm{h}(\rightarrow \mathrm{WW})+p_{\mathrm{T}}^{\text {miss }}$ and $\mathrm{h}(\rightarrow \mathrm{ZZ})+p_{\mathrm{T}}^{\text {miss }}$ channels}

The expected background yields and the observed number of event in data, along with the expected yields for two signal benchmarks in the $\mathrm{h} \rightarrow \mathrm{WW}$ and $\mathrm{h} \rightarrow \mathrm{ZZ}$ channels, are summarized in tables 6 and 7 , respectively.

Figure 5 shows the BDT discriminant distribution for the expected backgrounds and observed events in data for the $\mathrm{h} \rightarrow \mathrm{WW}$ analysis. Benchmark signal contributions in 


\begin{tabular}{|lcccc|}
\hline Channel & $4 \mathrm{e}$ & $4 \mu$ & $2 \mathrm{e} 2 \mu$ & $4 \ell$ \\
\hline SM Higgs boson $\left(m_{\mathrm{h}}=125 \mathrm{GeV}\right)$ & $12.1 \pm 1.4$ & $21.1 \pm 1.9$ & $27.9 \pm 2.4$ & $61.1 \pm 4.8$ \\
$\mathrm{Z} \gamma^{*}, \mathrm{ZZ}$ & $7.0_{-1.2}^{+0.9}$ & $14.7_{-1.2}^{+1.1}$ & $18.4_{-1.8}^{+1.7}$ & $40.1_{-3.6}^{+3.2}$ \\
$\mathrm{t} \overline{\mathrm{t}} \mathrm{V}$ & $0.10 \pm 0.02$ & $0.07 \pm 0.02$ & $0.12 \pm 0.02$ & $0.29 \pm 0.05$ \\
$\mathrm{VVV}$ & $0.04 \pm 0.03$ & - & $0.03 \pm 0.03$ & $0.07 \pm 0.06$ \\
$\mathrm{Z}+\mathrm{X}$ & $3.0 \pm 2.1$ & $4.7 \pm 2.7$ & $8.5 \pm 3.8$ & $16.2 \pm 4.9$ \\
Total bkg. & $22.2_{-2.8}^{+2.6}$ & $40.6 \pm 3.8$ & $55.0 \pm 4.8$ & $117.8_{-7.7}^{+7.5}$ \\
$\mathrm{Z}^{\prime}-2 \mathrm{HDM}\left(m_{\mathrm{Z}^{\prime}}=1200 \mathrm{GeV}, m_{\mathrm{A}}=300 \mathrm{GeV}\right)$ & $0.07 \pm 0.02$ & $0.11 \pm 0.02$ & $0.17 \pm 0.03$ & $0.36 \pm 0.06$ \\
Baryonic $\mathrm{Z}^{\prime}\left(m_{\mathrm{Z}^{\prime}}=500 \mathrm{GeV}, m_{\chi}=1 \mathrm{GeV}\right)$ & $0.25 \pm 0.06$ & $0.45 \pm 0.09$ & $0.67 \pm 0.14$ & $1.38 \pm 0.25$ \\
Observed & 24 & 44 & 44 & 112 \\
\hline
\end{tabular}

Table 7. The post-fit signal and background event yields, and the observed number of events in data, for the $\mathrm{h} \rightarrow \mathrm{ZZ}$ analysis. The expected numbers of signal events for the two signal hypotheses are also reported, one for each benchmark model. The total uncertainty, including both statistical and systematic components, is quoted for the expected signal and backgrounds yields.

the $\mathrm{Z}^{\prime}$-2HDM (left) and baryonic Z' (right) model are also shown, scaled by the factors of 500 and 100, respectively, for better visibility. Figure 6 shows the $p_{\mathrm{T}}^{\text {miss }}$ distribution of the expected backgrounds and observed events in data for the $\mathrm{h} \rightarrow \mathrm{ZZ}$ analysis. Benchmark signal contributions are also shown. For both analyses, the total uncertainty, given by a quadratic sum of the statistical and systematic components, is shown. The bottom panels show the ratios of data to the total background prediction with their total uncertainties.

The potential signal is extracted from the fit to the BDT discriminant $\left(p_{\mathrm{T}}^{\mathrm{miss}}\right)$ spectrum with a signal-plus-background hypothesis for the $\mathrm{h} \rightarrow \mathrm{WW}(\mathrm{h} \rightarrow \mathrm{ZZ})$ channel. The profile likelihood ratio is used as a test statistic, in an asymptotic approximation [93]. Data agree well with the expected background and no signal is observed in either channel. Limits on the model parameters at $95 \%$ confidence level (CL) are set using the modified frequentist $\mathrm{CL}_{\mathrm{s}}$ criterion [94-96] with all the nuisance parameters profiled.

The observed and expected upper limits on the DM candidate production cross section are shown in figure 7 for the $\mathrm{h} \rightarrow \mathrm{WW}$ (upper) and $\mathrm{h} \rightarrow \mathrm{ZZ}$ (lower) channels for the $\mathrm{Z}^{\prime}$ $2 \mathrm{HDM}$ with $m_{\mathrm{A}}=300 \mathrm{GeV}$ (left) and for the baryonic $\mathrm{Z}^{\prime}$ model with the value of $m_{\chi}$ fixed at $1 \mathrm{GeV}$ (right). All other model parameters are fixed to the values described in section 1. The upper limits for the $\mathrm{h} \rightarrow \mathrm{ZZ}$ analysis already include the statistical combination of all three final states used. The $\mathrm{h} \rightarrow \mathrm{WW}$ analysis excluded the region from 780 to $830 \mathrm{GeV}$ for $m_{\mathrm{A}}=300 \mathrm{GeV}$ in the $\mathrm{Z}^{\prime}-2 \mathrm{HDM}$.

\subsection{Results of the statistical combination}

The observed and expected upper limits at 95\% CL on the DM production cross section normalized to the predicted cross section, as a function of $m_{\mathrm{Z}^{\prime}}$, from the combination of all five channels are shown in figure 8 for the $\mathrm{Z}^{\prime}-2 \mathrm{HDM}$ with $m_{\mathrm{A}}=300 \mathrm{GeV}$ (left) and for the baryonic $\mathrm{Z}^{\prime}$ model with $m_{\chi}=1 \mathrm{GeV}$ (right). The combined result is also compared with those of the individual analyses. 

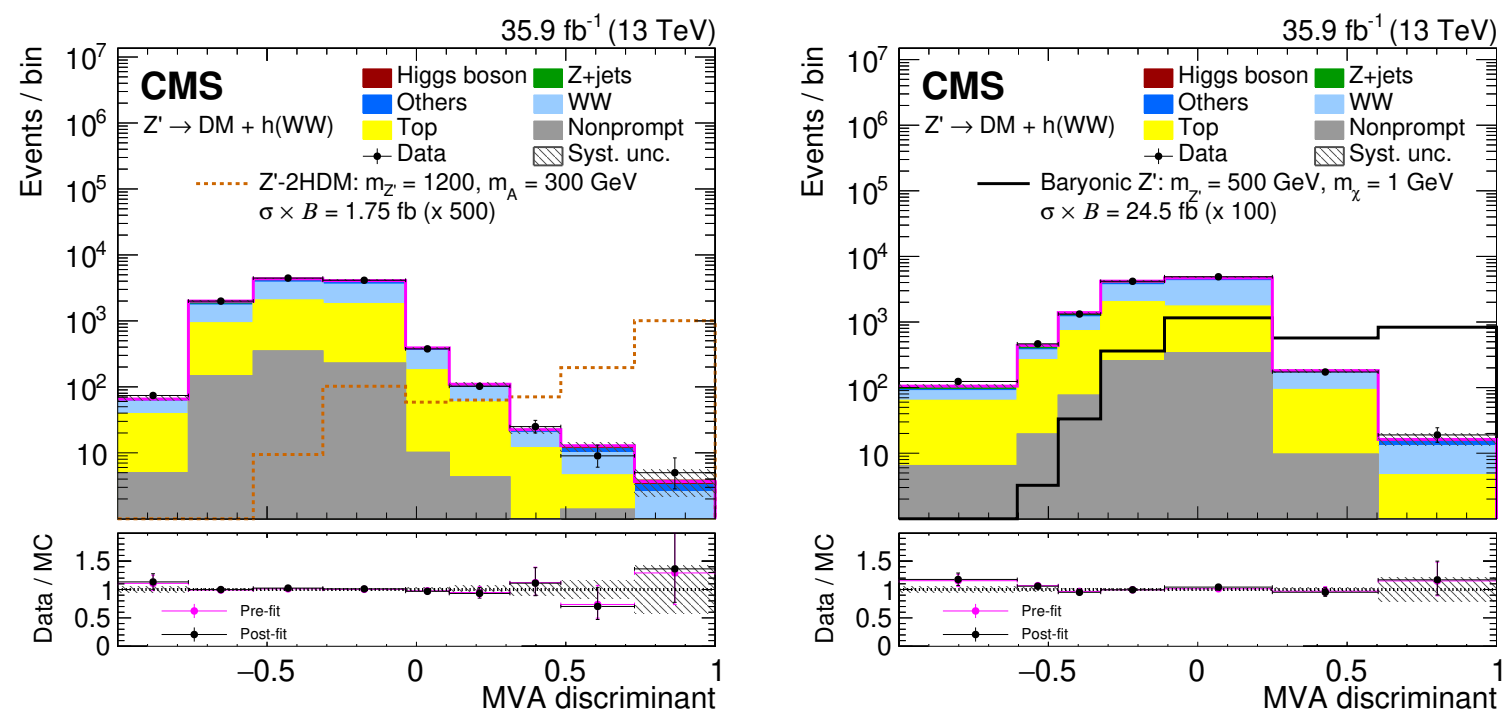

Figure 5. The distribution of the BDT discriminants expected from MC simulation before and after the fit, and observed in data (points with error bars) for the $\mathrm{Z}^{\prime}-2 \mathrm{HDM}$ (left) and baryonic $\mathrm{Z}^{\prime}$ (right) model in the signal region in the $\mathrm{h} \rightarrow \mathrm{WW}$ analysis. Two signal benchmarks, corresponding to the $\mathrm{Z}^{\prime}$-2HDM (dotted orange line, left) and baryonic $\mathrm{Z}^{\prime}$ (solid black line, right) model are superimposed. The signal is normalized to the product of cross section and $\mathcal{B}$, where $\mathcal{B}$ represents the $\mathrm{h} \rightarrow \mathrm{WW}$ branching fraction. The signal distributions are scaled up by a factor 500 (100) for the $\mathrm{Z}^{\prime}-2 \mathrm{HDM}$ (baryonic $\mathrm{Z}^{\prime}$ model), to make them more visible. The systematic uncertainties are shown by the hatched band. The lower panel shows the ratio of data to the total background yield, before and after the fit.

For the $\mathrm{Z}^{\prime}-2 \mathrm{HDM}$, the combination is dominated by the $\mathrm{h} \rightarrow$ bb analysis for $m_{\mathrm{Z}^{\prime}}>$ $800 \mathrm{GeV}$. However, the $\mathrm{h} \rightarrow$ bb analysis has no sensitivity for $m_{\mathrm{z}^{\prime}}$ values below $800 \mathrm{GeV}$, and a combination of the $\mathrm{h} \rightarrow \gamma \gamma$ and $\mathrm{h} \rightarrow \tau \tau$ channels plays a significant role in this region of the model parameter space. The range of $m_{\mathrm{z}^{\prime}}$ excluded at $95 \% \mathrm{CL}$ spans from 500 to $3200 \mathrm{GeV}$ for $m_{\mathrm{A}}=300 \mathrm{GeV}$.

For the baryonic $\mathrm{Z}^{\prime}$ model, the combination results are also dominated by the $\mathrm{h} \rightarrow \mathrm{bb}$ channel, but the $\mathrm{h} \rightarrow \gamma \gamma$ and $\mathrm{h} \rightarrow \tau \tau$ channels also provide a nonnegligible contribution in constraining the model parameters. The range of $m_{\mathrm{Z}^{\prime}}$ excluded at $95 \% \mathrm{CL}$ spans from 100 to $1600 \mathrm{GeV}$ for $m_{\chi}=1 \mathrm{GeV}$.

Figure 9 shows the observed and expected 95\% CL exclusion contours on $\sigma / \sigma_{\text {th }}$ in the $m_{\mathrm{Z}^{\prime}}-m_{\mathrm{A}}$ and $m_{\mathrm{Z}^{\prime}}-m_{\chi}$ planes for the $\mathrm{Z}^{\prime}-2 \mathrm{HDM}$ (left) and baryonic $\mathrm{Z}^{\prime}$ (right) model, respectively.

The results for the $\mathrm{Z}^{\prime}-2 \mathrm{HDM}$ are also interpreted in the $m_{\mathrm{Z}^{\prime}}-\tan \beta$ plane for three different $m_{\mathrm{A}}$ values: 300,400 , and $600 \mathrm{GeV}$. Since the shape of the $p_{\mathrm{T}}^{\text {miss }}$ distribution does not change with $\tan \beta$, and affects only the product of the $\mathrm{Z}^{\prime}$ production cross section and branching fraction to the mono-h channel, the limit shown in figure 9 (left) can be simply rescaled for different values of $\tan \beta$, from 0.5 to 10 . These limits, in the $m_{\mathrm{z}^{\prime}}-\tan \beta$ plane, are shown in figure 10. The area enclosed by the contour for a given value of $m_{\mathrm{A}}$ is excluded at $95 \%$ CL. 


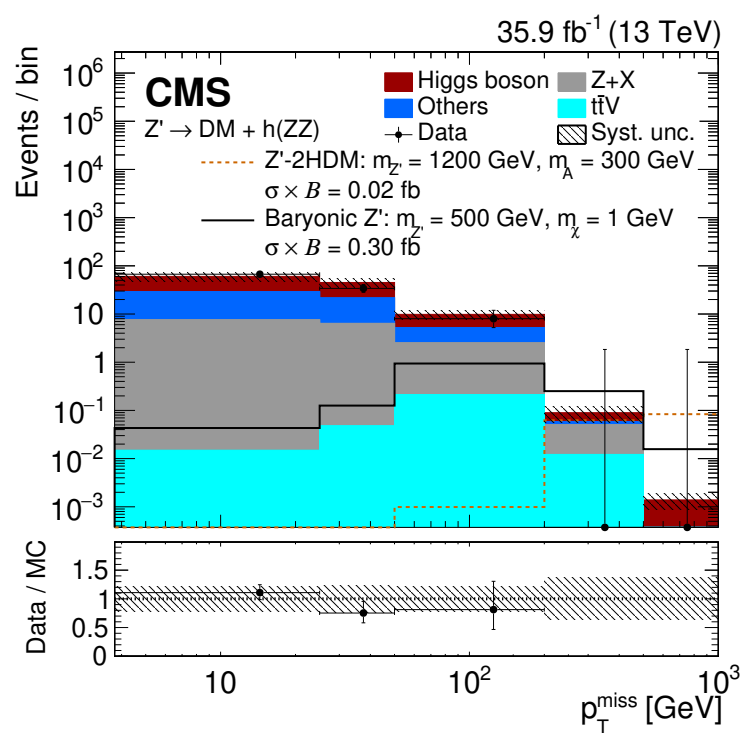

Figure 6. The $p_{\mathrm{T}}^{\text {miss }}$ distribution for the expected background and observed events in data in the $\mathrm{h} \rightarrow \mathrm{ZZ}$ analysis. Two signal benchmarks, corresponding to the $\mathrm{Z}^{\prime}-2 \mathrm{HDM}$ (dotted orange line, left) and baryonic $Z^{\prime}$ (solid black line, right) model are superimposed. The signal is normalized to the product of cross section and $\mathcal{B}$, where $\mathcal{B}$ represents the $\mathrm{h} \rightarrow \mathrm{ZZ}$ branching fraction. The systematic uncertainties are shown by the hatched band. The ratios of the data and the sum of all the SM backgrounds are shown in the bottom panels.

Limits for the baryonic $\mathrm{Z}^{\prime}$ model are also interpreted in terms of limits on the $s$ channel simplified DM model proposed by the ATLAS-CMS Dark Matter Forum [33] for comparison with direct-detection experiments. In this model, Dirac DM particles couple to a vector $\mathrm{Z}^{\prime}$ mediator, which also couples to the SM quarks. A point in the parameter space of this model is determined by four variables: the DM particle mass $m_{\chi}$, the mediator mass $m_{\text {med }}$, the mediator-DM coupling $g_{\chi}$, and the universal mediator-quark coupling $g_{\mathrm{q}}$. The couplings for the present analysis are fixed to $g_{\chi}=1.0$ and $g_{\mathrm{q}}=0.25$, following the recommendation of ref. [37]. The results are interpreted in terms of 90\% CL limits on the spin-independent (SI) cross section $\sigma^{\mathrm{SI}}$ for the DM-nucleon scattering. The value of $\sigma^{\mathrm{SI}}$ for a given set of parameters in the $s$-channel simplified DM model is given by [37]:

$$
\sigma^{\mathrm{SI}}=\frac{f^{2}\left(g_{\mathrm{q}}\right) g_{\chi}^{2} \mu_{\mathrm{nDM}}^{2}}{\pi m_{\mathrm{med}}^{4}},
$$

where $\mu_{\mathrm{nDM}}$ is the reduced mass of the DM-nucleon system and $f\left(g_{\mathrm{q}}\right)$ is the mediatornucleon coupling, which depends on $g_{\mathrm{q}}$. The resulting $\sigma^{\mathrm{SI}}$ limits, as functions of $m_{\chi}$ are shown in figure 11. Results obtained in this analysis are compared with those from the CMS dijet analyses ${ }^{1}[39,41]$ and from several direct-detection experiments. For the chosen set of parameters, the cross section limit from the present analysis is more stringent than the direct-detection limits for $m_{\chi}$ between 1 and $5 \mathrm{GeV}$.

\footnotetext{
${ }^{1}$ We note that the limits presented in [39] are at 95\% CL, while the corresponding results at $90 \%$ CL from that analysis are shown here.
} 

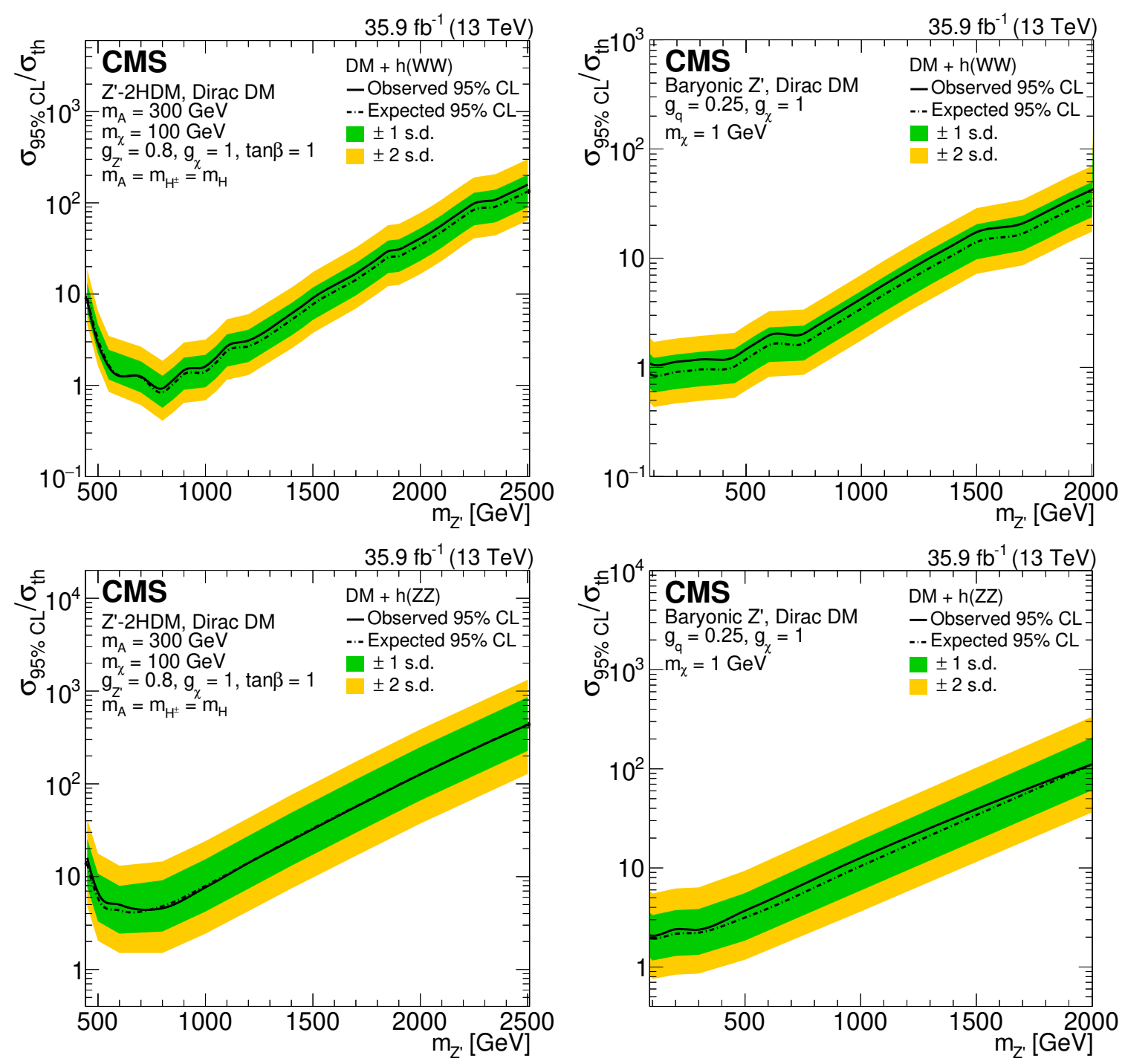

Figure 7. The upper limits at 95\% CL on the observed and expected DM production cross section for the $\mathrm{h} \rightarrow \mathrm{WW}$ (upper) and $\mathrm{h} \rightarrow \mathrm{ZZ}$ (lower) analyses for the $\mathrm{Z}^{\prime}-2 \mathrm{HDM}$ with $m_{\mathrm{A}}=300 \mathrm{GeV}$ (left) and for the baryonic $\mathrm{Z}^{\prime}$ with $m_{\chi}=1 \mathrm{GeV}$ (right) model. The inner and outer shaded bands show the 68 and $95 \%$ uncertainties in the expected limit, respectively.

\section{Summary}

A search for dark matter particles produced in association with a Higgs boson has been presented, using a sample of proton-proton collision data at a center-of-mass energy of $13 \mathrm{TeV}$, corresponding to an integrated luminosity of $35.9 \mathrm{fb}^{-1}$. Results from five decay channels of the Higgs boson, $\mathrm{h} \rightarrow \mathrm{b} \overline{\mathrm{b}}, \mathrm{h} \rightarrow \gamma \gamma, \mathrm{h} \rightarrow \tau^{+} \tau^{-}, \mathrm{h} \rightarrow \mathrm{W}^{+} \mathrm{W}^{-}$, and $\mathrm{h} \rightarrow \mathrm{ZZ}$, are described, along with their statistical combination. No significant deviation from the standard model prediction is observed in any of the channels or in their combination. Upper limits at $95 \%$ confidence level on the production cross section of dark matter are set in a type-II two Higgs doublet model extended by a $\mathrm{Z}^{\prime}$ boson and in a baryonic $\mathrm{Z}^{\prime}$ model. 

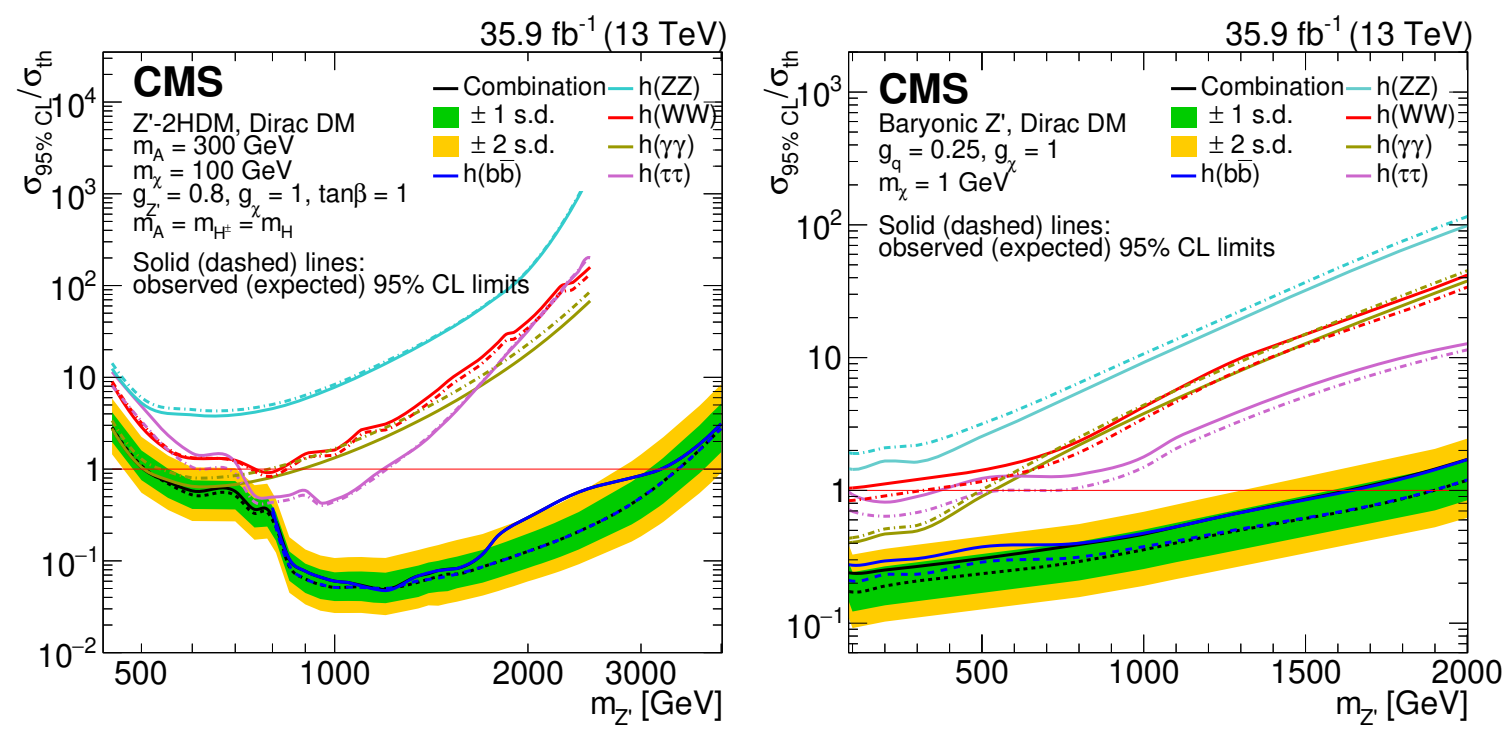

Figure 8. The upper limits at 95\% CL on the observed and expected $\sigma / \sigma_{\text {th }}$ for the $\mathrm{Z}^{\prime}-2 \mathrm{HDM}$ (left) and baryonic $Z^{\prime}$ (right) model for the five individual decay modes of the Higgs boson, and for their combination. The distributions are shown as a function of $m_{\mathrm{z}^{\prime}}$ for $m_{\mathrm{A}}=300 \mathrm{GeV}\left(\mathrm{Z}^{\prime}-2 \mathrm{HDM}\right)$ and $m_{\chi}=1 \mathrm{GeV}$ (baryonic $\mathrm{Z}^{\prime}$ model). The inner and outer shaded bands show the 68 and $95 \% \mathrm{CL}$ uncertainties in the expected limit, respectively.
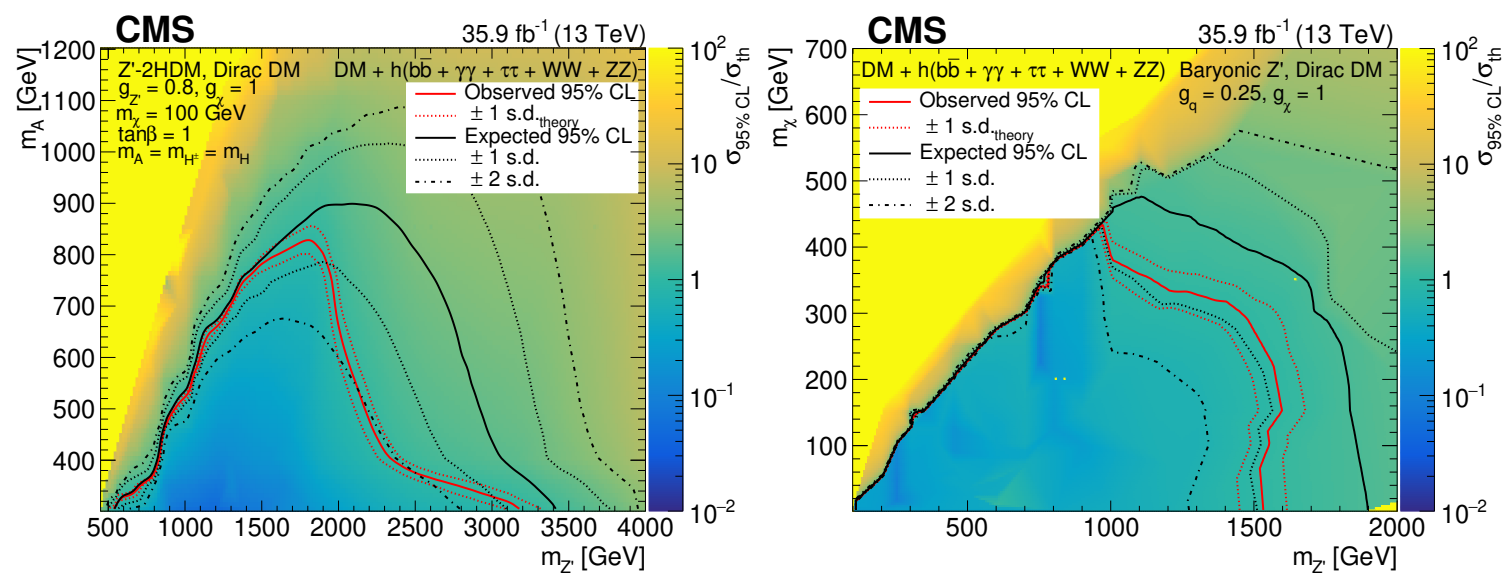

Figure 9. The upper limits at $95 \% \mathrm{CL}$ on the observed and expected $\sigma / \sigma_{\mathrm{th}}$ in the $m_{\mathrm{z}^{\prime}}-m_{\mathrm{A}}$ and $m_{\mathrm{Z}^{\prime}}-m_{\chi}$ planes for the $\mathrm{Z}^{\prime}-2 \mathrm{HDM}$ (left) and baryonic $\mathrm{Z}^{\prime}$ model (right), respectively. The region enclosed by the contours is excluded using the combination of the five decay channels of the Higgs boson for the following benchmark scenarios: $g_{\mathrm{Z}^{\prime}}=0.8, g_{\chi}=1, \tan \beta=1, m_{\chi}=100 \mathrm{GeV}$, and $m_{\mathrm{A}}=m_{\mathrm{H}}=m_{\mathrm{H}^{ \pm}}$for the $\mathrm{Z}^{\prime}-2 \mathrm{HDM}$, and $g_{\chi}=1, g_{\mathrm{q}}=0.25$ for the baryonic $\mathrm{Z}^{\prime}$ model. 


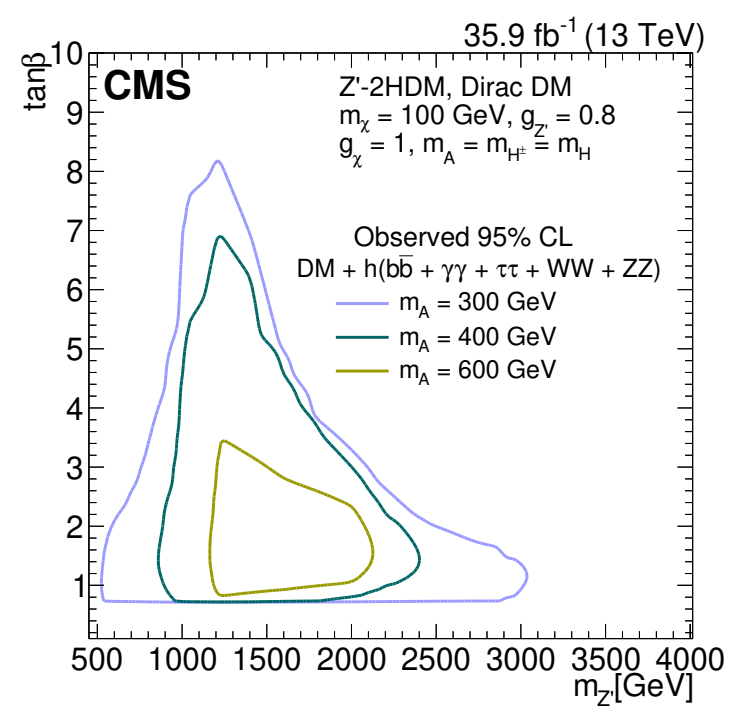

Figure 10. The upper limits at $95 \% \mathrm{CL}$ on the observed $\sigma / \sigma_{\mathrm{th}}$ for the $\mathrm{Z}^{\prime}-2 \mathrm{HDM}$ in the $m_{\mathrm{Z}^{\prime}}-\tan \beta$ plane from the combination of the five Higgs boson decay channels. Each contour represents the excluded region for a given value of $m_{\mathrm{A}}=300,400$, and $600 \mathrm{GeV}$.

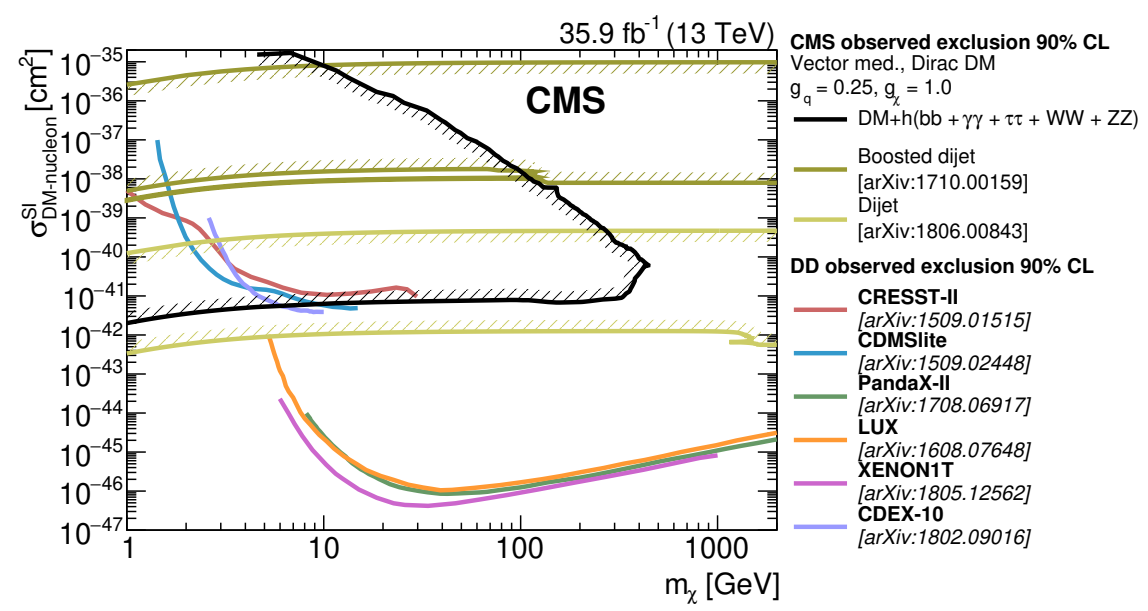

Figure 11. The upper limits at $90 \%$ CL on the DM-nucleon spin-independent scattering cross section $\sigma^{\mathrm{SI}}$, as a function of $m_{\chi}$. Results obtained in this analysis are compared with those from the CMS dijet analyses [39, 41] and from several direct-detection experiments: CRESST-II [97], CDMSLite [98], PandaX-II [99], LUX [100], XENON-1T [101], and CDEX-10 [102].

The results in the baryonic $\mathrm{Z}^{\prime}$ model are also interpreted in terms of the spin-independent dark matter nucleon scattering cross section. This is the first search for DM particles produced in association with a Higgs boson decaying to a pair of $\mathrm{W}$ or $\mathrm{Z}$ bosons, and the first statistical combination based on five Higgs boson decay channels.

\section{Acknowledgments}

We congratulate our colleagues in the CERN accelerator departments for the excellent performance of the LHC and thank the technical and administrative staffs at CERN and at other CMS institutes for their contributions to the success of the CMS effort. In addition, 
we gratefully acknowledge the computing centers and personnel of the Worldwide LHC Computing Grid for delivering so effectively the computing infrastructure essential to our analyses. Finally, we acknowledge the enduring support for the construction and operation of the LHC and the CMS detector provided by the following funding agencies: BMBWF and FWF (Austria); FNRS and FWO (Belgium); CNPq, CAPES, FAPERJ, FAPERGS, and FAPESP (Brazil); MES (Bulgaria); CERN; CAS, MoST, and NSFC (China); COLCIENCIAS (Colombia); MSES and CSF (Croatia); RPF (Cyprus); SENESCYT (Ecuador); MoER, ERC IUT, PUT and ERDF (Estonia); Academy of Finland, MEC, and HIP (Finland); CEA and CNRS/IN2P3 (France); BMBF, DFG, and HGF (Germany); GSRT (Greece); NKFIA (Hungary); DAE and DST (India); IPM (Iran); SFI (Ireland); INFN (Italy); MSIP and NRF (Republic of Korea); MES (Latvia); LAS (Lithuania); MOE and UM (Malaysia); BUAP, CINVESTAV, CONACYT, LNS, SEP, and UASLP-FAI (Mexico); MOS (Montenegro); MBIE (New Zealand); PAEC (Pakistan); MSHE and NSC (Poland); FCT (Portugal); JINR (Dubna); MON, RosAtom, RAS, RFBR, and NRC KI (Russia); MESTD (Serbia); SEIDI, CPAN, PCTI, and FEDER (Spain); MOSTR (Sri Lanka); Swiss Funding Agencies (Switzerland); MST (Taipei); ThEPCenter, IPST, STAR, and NSTDA (Thailand); TUBITAK and TAEK (Turkey); NASU and SFFR (Ukraine); STFC (United Kingdom); DOE and NSF (U.S.A.).

Individuals have received support from the Marie-Curie program and the European Research Council and Horizon 2020 Grant, contract Nos. 675440, 752730, and 765710 (European Union); the Leventis Foundation; the A.P. Sloan Foundation; the Alexander von Humboldt Foundation; the Belgian Federal Science Policy Office; the Fonds pour la Formation à la Recherche dans l'Industrie et dans l'Agriculture (FRIA-Belgium); the Agentschap voor Innovatie door Wetenschap en Technologie (IWT-Belgium); the F.R.S.-FNRS and FWO (Belgium) under the "Excellence of Science - EOS" — be.h project n. 30820817; the Beijing Municipal Science \& Technology Commission, No. Z181100004218003; the Ministry of Education, Youth and Sports (MEYS) of the Czech Republic; the Lendület ("Momentum") Program and the János Bolyai Research Scholarship of the Hungarian Academy of Sciences, the New National Excellence Program ÚNKP, the NKFIA research grants 123842, 123959, 124845, 124850, 125105, 128713, 128786, and 129058 (Hungary); the Council of Science and Industrial Research, India; the HOMING PLUS program of the Foundation for Polish Science, cofinanced from European Union, Regional Development Fund, the Mobility Plus program of the Ministry of Science and Higher Education, the National Science Center (Poland), contracts Harmonia 2014/14/M/ST2/00428, Opus 2014/13/B/ST2/02543, 2014/15/B/ST2/03998, and 2015/19/B/ST2/02861, Sonatabis 2012/07/E/ST2/01406; the National Priorities Research Program by Qatar National Research Fund; the Ministry of Science and Education, grant no. 3.2989.2017 (Russia); the Programa Estatal de Fomento de la Investigación Científica y Técnica de Excelencia María de Maeztu, grant MDM-2015-0509 and the Programa Severo Ochoa del Principado de Asturias; the Thalis and Aristeia programs cofinanced by EU-ESF and the Greek NSRF; the Rachadapisek Sompot Fund for Postdoctoral Fellowship, Chulalongkorn University and the Chulalongkorn Academic into Its 2nd Century Project Advancement Project (Thailand); the Welch Foundation, contract C-1845; and the Weston Havens Foundation (U.S.A.). 
Open Access. This article is distributed under the terms of the Creative Commons Attribution License (CC-BY 4.0), which permits any use, distribution and reproduction in any medium, provided the original author(s) and source are credited.

\section{References}

[1] R.J. Gaitskell, Direct detection of dark matter, Ann. Rev. Nucl. Part. Sci. 54 (2004) 315 [INSPIRE].

[2] V. Trimble, Existence and Nature of Dark Matter in the Universe, Ann. Rev. Astron. Astrophys. 25 (1987) 425 [INSPIRE].

[3] T.A. Porter, R.P. Johnson and P.W. Graham, Dark Matter Searches with Astroparticle Data, Ann. Rev. Astron. Astrophys. 49 (2011) 155 [arXiv:1104.2836] [INSPIRE].

[4] G. Bertone, D. Hooper and J. Silk, Particle dark matter: Evidence, candidates and constraints, Phys. Rept. 405 (2005) 279 [hep-ph/0404175] [INSPIRE].

[5] Planck collaboration, Planck 2018 results. I. Overview and the cosmological legacy of Planck, arXiv:1807.06205 [INSPIRE].

[6] J.L. Feng, Dark Matter Candidates from Particle Physics and Methods of Detection, Ann. Rev. Astron. Astrophys. 48 (2010) 495 [arXiv: 1003. 0904] [INSPIRE].

[7] R.J. Scherrer and M.S. Turner, On the Relic, Cosmic Abundance of Stable Weakly Interacting Massive Particles, Phys. Rev. D 33 (1986) 1585 [Erratum ibid. D 34 (1986) 3263] [INSPIRE].

[8] G. Steigman and M.S. Turner, Cosmological Constraints on the Properties of Weakly Interacting Massive Particles, Nucl. Phys. B 253 (1985) 375 [inSPIRE].

[9] M. Beltrán, D. Hooper, E.W. Kolb, Z.A.C. Krusberg and T.M.P. Tait, Maverick dark matter at colliders, JHEP 09 (2010) 037 [arXiv: 1002.4137] [InSPIRE].

[10] ATLAS collaboration, Search for dark matter and other new phenomena in events with an energetic jet and large missing transverse momentum using the ATLAS detector, JHEP 01 (2018) 126 [arXiv:1711.03301] [INSPIRE].

[11] CMS collaboration, Search for new physics in final states with an energetic jet or a hadronically decaying $W$ or $Z$ boson and transverse momentum imbalance at $\sqrt{s}=13 \mathrm{TeV}$, Phys. Rev. D 97 (2018) 092005 [arXiv:1712.02345] [INSPIRE].

[12] ATLAS collaboration, Search for dark matter produced in association with bottom or top quarks in $\sqrt{s}=13 \mathrm{TeV}$ pp collisions with the ATLAS detector, Eur. Phys. J. C 78 (2018) 18 [arXiv: 1710.11412] [INSPIRE].

[13] CMS collaboration, Search for dark matter in events with energetic, hadronically decaying top quarks and missing transverse momentum at $\sqrt{s}=13 \mathrm{TeV}$, JHEP 06 (2018) 027 [arXiv: 1801.08427] [INSPIRE].

[14] ATLAS collaboration, Search for dark matter at $\sqrt{s}=13 \mathrm{TeV}$ in final states containing an energetic photon and large missing transverse momentum with the ATLAS detector, Eur. Phys. J. C 77 (2017) 393 [arXiv:1704.03848] [INSPIRE].

[15] CMS collaboration, Search for new physics in the monophoton final state in proton-proton collisions at $\sqrt{s}=13 \mathrm{TeV}$, JHEP 10 (2017) 073 [arXiv: 1706.03794] [INSPIRE]. 
[16] ATLAS collaboration, Search for an invisibly decaying Higgs boson or dark matter candidates produced in association with a $Z$ boson in pp collisions at $\sqrt{s}=13 \mathrm{TeV}$ with the ATLAS detector, Phys. Lett. B 776 (2018) 318 [arXiv:1708.09624] [inSPIRE].

[17] CMS collaboration, Search for new physics in events with a leptonically decaying $Z$ boson and a large transverse momentum imbalance in proton-proton collisions at $\sqrt{s}=13 \mathrm{TeV}$, Eur. Phys. J. C 78 (2018) 291 [arXiv:1711.00431] [InSPIRE].

[18] ATLAS collaboration, Search for dark matter in events with a hadronically decaying vector boson and missing transverse momentum in pp collisions at $\sqrt{s}=13 \mathrm{TeV}$ with the ATLAS detector, JHEP 10 (2018) 180 [arXiv:1807.11471] [INSPIRE].

[19] ATLAS collaboration, Observation of a new particle in the search for the Standard Model Higgs boson with the ATLAS detector at the LHC, Phys. Lett. B 716 (2012) 1 [arXiv: 1207.7214] [INSPIRE].

[20] CMS collaboration, Observation of a New Boson at a Mass of $125 \mathrm{GeV}$ with the CMS Experiment at the LHC, Phys. Lett. B 716 (2012) 30 [arXiv:1207.7235] [INSPIRE].

[21] CMS collaboration, Observation of a New Boson with Mass Near $125 \mathrm{GeV}$ in pp Collisions at $\sqrt{s}=7$ and $8 \mathrm{TeV}$, JHEP 06 (2013) 081 [arXiv:1303.4571] [INSPIRE].

[22] L. Carpenter, A. DiFranzo, M. Mulhearn, C. Shimmin, S. Tulin and D. Whiteson, Mono-Higgs-boson: A new collider probe of dark matter, Phys. Rev. D 89 (2014) 075017 [arXiv:1312.2592] [INSPIRE].

[23] J.M. No, Looking through the pseudoscalar portal into dark matter: Novel mono-Higgs and mono-Z signatures at the LHC, Phys. Rev. D 93 (2016) 031701 [arXiv:1509.01110] [INSPIRE].

[24] ATLAS collaboration, Search for Dark Matter in Events with Missing Transverse Momentum and a Higgs Boson Decaying to Two Photons in pp Collisions at $\sqrt{s}=8 \mathrm{TeV}$ with the ATLAS Detector, Phys. Rev. Lett. 115 (2015) 131801 [arXiv:1506.01081] [INSPIRE].

[25] ATLAS collaboration, Search for dark matter produced in association with a Higgs boson decaying to two bottom quarks in pp collisions at $\sqrt{s}=8 \mathrm{TeV}$ with the ATLAS detector, Phys. Rev. D 93 (2016) 072007 [arXiv: 1510.06218] [InSPIRE].

[26] ATLAS collaboration, Search for dark matter in association with a Higgs boson decaying to b-quarks in pp collisions at $\sqrt{s}=13$ TeV with the ATLAS detector, Phys. Lett. B 765 (2017) 11 [arXiv:1609.04572] [INSPIRE].

[27] CMS collaboration, Search for associated production of dark matter with a Higgs boson decaying to $b \bar{b}$ or $\gamma \gamma$ at $\sqrt{s}=13 \mathrm{TeV}$, JHEP 10 (2017) 180 [arXiv:1703.05236] [INSPIRE].

[28] ATLAS collaboration, Search for dark matter in association with a Higgs boson decaying to two photons at $\sqrt{s}=13 \mathrm{TeV}$ with the ATLAS detector, Phys. Rev. D 96 (2017) 112004 [arXiv: 1706. 03948] [INSPIRE].

[29] ATLAS collaboration, Search for Dark Matter Produced in Association with a Higgs Boson Decaying to b $\bar{b}$ using $36 \mathrm{fb}^{-1}$ of $\mathrm{pp}$ collisions at $\sqrt{\mathrm{s}}=13 \mathrm{TeV}$ with the ATLAS Detector, Phys. Rev. Lett. 119 (2017) 181804 [arXiv:1707.01302] [INSPIRE].

[30] CMS collaboration, Search for heavy resonances decaying into a vector boson and a Higgs boson in final states with charged leptons, neutrinos and $b$ quarks at $\sqrt{s}=13 \mathrm{TeV}$, JHEP 11 (2018) 172 [arXiv: 1807.02826] [INSPIRE]. 
[31] CMS collaboration, Search for dark matter produced in association with a Higgs boson decaying to a pair of bottom quarks in proton-proton collisions at $\sqrt{s}=13 \mathrm{TeV}$, Eur. Phys. J. C 79 (2019) 280 [arXiv:1811.06562] [INSPIRE].

[32] CMS collaboration, Search for dark matter produced in association with a Higgs boson decaying to $\gamma \gamma$ or $\tau^{+} \tau^{-}$at $\sqrt{s}=13 \mathrm{TeV}$, JHEP 09 (2018) 046 [arXiv:1806.04771] [INSPIRE].

[33] D. Abercrombie et al., Dark Matter Benchmark Models for Early LHC Run-2 Searches: Report of the ATLAS/CMS Dark Matter Forum, Phys. Dark Univ. 26 (2019) 100371 [FERMILAB-PUB-15-282] [arXiv: 1507.00966] [INSPIRE].

[34] G.C. Branco, P.M. Ferreira, L. Lavoura, M.N. Rebelo, M. Sher and J.P. Silva, Theory and phenomenology of two-Higgs-doublet models, Phys. Rept. 516 (2012) 1 [arXiv:1106.0034] [INSPIRE].

[35] N. Craig, J. Galloway and S. Thomas, Searching for Signs of the Second Higgs Doublet, arXiv:1305.2424 [INSPIRE].

[36] A. Berlin, T. Lin and L.-T. Wang, Mono-Higgs Detection of Dark Matter at the LHC, JHEP 06 (2014) 078 [arXiv: 1402.7074] [InSPIRE].

[37] G. Busoni et al., Recommendations on presenting LHC searches for missing transverse energy signals using simplified s-channel models of dark matter, in press Phys. Dark Univ. (2019) [arXiv: 1603.04156] [INSPIRE].

[38] ATLAS collaboration, Search for new phenomena in dijet events using $37 \mathrm{fb}^{-1}$ of $\mathrm{pp}$ collision data collected at $\sqrt{s}=13 \mathrm{TeV}$ with the ATLAS detector, Phys. Rev. D 96 (2017) 052004 [arXiv: 1703.09127] [INSPIRE].

[39] CMS collaboration, Search for low mass vector resonances decaying into quark-antiquark pairs in proton-proton collisions at $\sqrt{s}=13 \mathrm{TeV}, \mathrm{JHEP} 01$ (2018) 097 [arXiv:1710.00159] [INSPIRE].

[40] ATLAS collaboration, Search for low-mass dijet resonances using trigger-level jets with the ATLAS detector in pp collisions at $\sqrt{s}=13$ TeV, Phys. Rev. Lett. 121 (2018) 081801 [arXiv: 1804.03496] [INSPIRE].

[41] CMS collaboration, Search for narrow and broad dijet resonances in proton-proton collisions at $\sqrt{s}=13 \mathrm{TeV}$ and constraints on dark matter mediators and other new particles, JHEP 08 (2018) 130 [arXiv:1806.00843] [INSPIRE].

[42] CMS collaboration, Measurements of properties of the Higgs boson decaying into the four-lepton final state in pp collisions at $\sqrt{s}=13$ TeV, JHEP 11 (2017) 047 [arXiv: 1706. 09936] [INSPIRE].

[43] CMS collaboration, The CMS trigger system, 2017 JINST 12 P01020 [arXiv:1609.02366] [INSPIRE].

[44] CMS collaboration, The CMS Experiment at the CERN LHC, 2008 JINST 3 S08004 [INSPIRE].

[45] CMS collaboration, Measurement of the inelastic proton-proton cross section at $\sqrt{s}=13 \mathrm{TeV}, \mathrm{JHEP} 07$ (2018) 161 [arXiv: 1802.02613] [INSPIRE].

[46] J. Alwall, M. Herquet, F. Maltoni, O. Mattelaer and T. Stelzer, MadGraph 5: Going Beyond, JHEP 06 (2011) 128 [arXiv:1106.0522] [INSPIRE]. 
[47] J. Alwall et al., The automated computation of tree-level and next-to-leading order differential cross sections and their matching to parton shower simulations, JHEP $\mathbf{0 7}$ (2014) 079 [arXiv: 1405.0301] [INSPIRE].

[48] T. Sjöstrand et al., An Introduction to PYTHIA 8.2, Comput. Phys. Commun. 191 (2015) 159 [arXiv: 1410.3012] [INSPIRE].

[49] P. Nason, A New method for combining NLO QCD with shower Monte Carlo algorithms, JHEP 11 (2004) 040 [hep-ph/0409146] [INSPIRE].

[50] S. Frixione, P. Nason and C. Oleari, Matching NLO QCD computations with Parton Shower simulations: the POWHEG method, JHEP 11 (2007) 070 [arXiv:0709.2092] [INSPIRE].

[51] S. Alioli, P. Nason, C. Oleari and E. Re, A general framework for implementing NLO calculations in shower Monte Carlo programs: the POWHEG BOX, JHEP 06 (2010) 043 [arXiv: 1002 .2581] [INSPIRE].

[52] T. Melia, P. Nason, R. Röntsch and G. Zanderighi, $W^{+} W^{-}, W Z$ and $Z Z$ production in the POWHEG BOX, JHEP 11 (2011) 078 [arXiv:1107.5051] [INSPIRE].

[53] K. Melnikov and F. Petriello, Electroweak gauge boson production at hadron colliders through $O\left(\alpha_{s}^{2}\right)$, Phys. Rev. D 74 (2006) 114017 [hep-ph/0609070] [INSPIRE].

[54] J.M. Campbell, R.K. Ellis and C. Williams, Vector boson pair production at the LHC, JHEP 07 (2011) 018 [arXiv:1105.0020] [INSPIRE].

[55] J.M. Campbell, R.K. Ellis and W.T. Giele, A Multi-Threaded Version of MCFM, Eur. Phys. J. C 75 (2015) 246 [arXiv:1503.06182] [InSPIRE].

[56] P. Meade, H. Ramani and M. Zeng, Transverse momentum resummation effects in $W^{+} W^{-}$ measurements, Phys. Rev. D 90 (2014) 114006 [arXiv:1407.4481] [INSPIRE].

[57] P. Jaiswal and T. Okui, Explanation of the $W W$ excess at the LHC by jet-veto resummation, Phys. Rev. D 90 (2014) 073009 [arXiv: 1407.4537] [inSPIRE].

[58] F. Caola, K. Melnikov, R. Röntsch and L. Tancredi, QCD corrections to $W^{+} W^{-}$production through gluon fusion, Phys. Lett. B 754 (2016) 275 [arXiv:1511.08617] [INSPIRE].

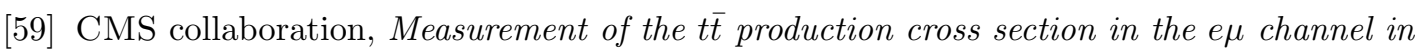
proton-proton collisions at $\sqrt{s}=7$ and 8 TeV, JHEP 08 (2016) 029 [arXiv:1603.02303] [INSPIRE].

[60] S. Alioli, P. Nason, C. Oleari and E. Re, NLO vector-boson production matched with shower in POWHEG, JHEP 07 (2008) 060 [arXiv:0805.4802] [INSPIRE].

[61] E. Bagnaschi, G. Degrassi, P. Slavich and A. Vicini, Higgs production via gluon fusion in the POWHEG approach in the SM and in the MSSM, JHEP 02 (2012) 088 [arXiv:1111.2854] [INSPIRE].

[62] P. Nason and C. Oleari, NLO Higgs boson production via vector-boson fusion matched with shower in POWHEG, JHEP 02 (2010) 037 [arXiv:0911.5299] [INSPIRE].

[63] H.B. Hartanto, B. Jager, L. Reina and D. Wackeroth, Higgs boson production in association with top quarks in the POWHEG BOX, Phys. Rev. D 91 (2015) 094003 [arXiv: 1501.04498] [INSPIRE].

[64] G. Luisoni, P. Nason, C. Oleari and F. Tramontano, $H W^{ \pm} / H Z+0$ and 1 jet at $N L O$ with the POWHEG BOX interfaced to GoSam and their merging within MiNLO, JHEP 10 (2013) 083 [arXiv: 1306.2542] [INSPIRE]. 
[65] Y. Gao, A.V. Gritsan, Z. Guo, K. Melnikov, M. Schulze and N.V. Tran, Spin Determination of Single-Produced Resonances at Hadron Colliders, Phys. Rev. D 81 (2010) 075022 [arXiv:1001.3396] [INSPIRE].

[66] S. Bolognesi et al., On the spin and parity of a single-produced resonance at the LHC, Phys. Rev. D 86 (2012) 095031 [arXiv: 1208.4018] [inSPIRE].

[67] LHC Higgs Cross Section Working Group, Handbook of LHC Higgs Cross Sections: 4. Deciphering the Nature of the Higgs Sector, CERN Yellow Rep. Monogr. 2 (2017) 1 [arXiv: 1610.07922] [INSPIRE].

[68] NNPDF collaboration, Parton distributions for the LHC Run II, JHEP 04 (2015) 040 [arXiv: 1410.8849] [INSPIRE].

[69] CMS collaboration, Event generator tunes obtained from underlying event and multiparton scattering measurements, Eur. Phys. J. C 76 (2016) 155 [arXiv:1512.00815] [INSPIRE].

[70] GEANT4 collaboration, GEANT4: A Simulation toolkit, Nucl. Instrum. Meth. A 506 (2003) 250 [INSPIRE].

[71] CMS collaboration, Particle-flow reconstruction and global event description with the CMS detector, 2017 JINST 12 P10003 [arXiv:1706. 04965] [INSPIRE].

[72] CMS collaboration, Performance of Electron Reconstruction and Selection with the CMS Detector in Proton-Proton Collisions at $\sqrt{s}=8 \mathrm{TeV}, 2015$ JINST $10 \mathrm{P} 06005$ [arXiv: 1502 . 02701] [INSPIRE].

[73] M. Cacciari and G.P. Salam, Dispelling the $N^{3}$ myth for the $k_{t}$ jet-finder, Phys. Lett. B 641 (2006) 57 [hep-ph/0512210] [INSPIRE].

[74] M. Cacciari, G.P. Salam and G. Soyez, FastJet User Manual, Eur. Phys. J. C 72 (2012) 1896 [arXiv:1111.6097] [INSPIRE].

[75] M. Cacciari and G.P. Salam, Pileup subtraction using jet areas, Phys. Lett. B 659 (2008) 119 [arXiv: 0707.1378] [INSPIRE].

[76] CMS collaboration, Jet energy scale and resolution in the CMS experiment in pp collisions at $8 \mathrm{TeV}, 2017$ JINST $12 \mathrm{P} 02014$ [arXiv:1607.03663] [INSPIRE].

[77] CMS collaboration, Jet Performance in pp Collisions at 7 TeV, CMS-PAS-JME-10-003 (2010) [INSPIRE].

[78] M. Wobisch and T. Wengler, Hadronization corrections to jet cross-sections in deep inelastic scattering, in proceedings of the Workshop on Monte Carlo Generators for HERA Physics (Plenary Starting Meeting), Hamburg, Germany, 27-30 April 1998, pp. 270-279 [hep-ph/9907280] [INSPIRE].

[79] CMS collaboration, A Cambridge-Aachen (C-A) based Jet Algorithm for boosted top-jet tagging, CMS-PAS-JME-09-001 (2009) [INSPIRE].

[80] D. Bertolini, P. Harris, M. Low and N. Tran, Pileup Per Particle Identification, JHEP 10 (2014) 059 [arXiv: 1407.6013] [INSPIRE].

[81] A.J. Larkoski, S. Marzani, G. Soyez and J. Thaler, Soft Drop, JHEP 05 (2014) 146 [arXiv: 1402.2657] [INSPIRE].

[82] CMS collaboration, Identification of heavy-flavour jets with the CMS detector in $p p$ collisions at $13 \mathrm{TeV}, 2018$ JINST 13 P05011 [arXiv: 1712.07158] [INSPIRE]. 
[83] CMS collaboration, Performance of reconstruction and identification of $\tau$ leptons decaying to hadrons and $\nu_{\tau}$ in pp collisions at $\sqrt{s}=13 \mathrm{TeV}, 2018$ JINST $13 \mathrm{P} 10005$ [arXiv: 1809.02816] [INSPIRE].

[84] CMS collaboration, Performance of missing transverse momentum reconstruction in proton-proton collisions at $\sqrt{s}=13 \mathrm{TeV}$ using the CMS detector, 2019 JINST $14 \mathrm{P} 07004$ [arXiv: 1903.06078] [INSPIRE].

[85] CMS collaboration, Measurements of properties of the Higgs boson decaying to a $W$ boson pair in pp collisions at $\sqrt{s}=13 \mathrm{TeV}$, Phys. Lett. B 791 (2019) 96 [arXiv:1806.05246] [INSPIRE].

[86] Particle Data Group, Review of Particle Physics, Phys. Rev. D 98 (2018) 030001 [INSPIRE].

[87] CMS collaboration, Measurement of Higgs Boson Production and Properties in the $W W$ Decay Channel with Leptonic Final States, JHEP 01 (2014) 096 [arXiv:1312.1129] [INSPIRE].

[88] CMS collaboration, Measurements of differential cross sections of top quark pair production as a function of kinematic event variables in proton-proton collisions at $\sqrt{s}=13 \mathrm{TeV}$, JHEP 06 (2018) 002 [arXiv:1803.03991] [INSPIRE].

[89] G. Passarino, Higgs CAT, Eur. Phys. J. C 74 (2014) 2866 [arXiv:1312.2397] [inSPIRE].

[90] S. Frixione, V. Hirschi, D. Pagani, H.S. Shao and M. Zaro, Electroweak and QCD corrections to top-pair hadroproduction in association with heavy bosons, JHEP 06 (2015) 184 [arXiv: 1504.03446] [INSPIRE].

[91] CMS collaboration, CMS Luminosity Measurements for the 2016 Data Taking Period, CMS-PAS-LUM-17-001 (2017) [INSPIRE].

[92] J. Butterworth et al., PDF4LHC recommendations for LHC Run II, J. Phys. G 43 (2016) 023001 [arXiv: 1510.03865] [INSPIRE].

[93] G. Cowan, K. Cranmer, E. Gross and O. Vitells, Asymptotic formulae for likelihood-based tests of new physics, Eur. Phys. J. C 71 (2011) 1554 [Erratum ibid. C 73 (2013) 2501] [arXiv: 1007.1727] [INSPIRE].

[94] T. Junk, Confidence level computation for combining searches with small statistics, Nucl. Instrum. Meth. A 434 (1999) 435 [hep-ex/9902006] [INSPIRE].

[95] A.L. Read, Presentation of search results: The $C L_{s}$ technique, J. Phys. G 28 (2002) 2693 [INSPIRE].

[96] ATLAS, CMS collaborations and the LHC Higgs Combination Group, Procedure for the LHC Higgs boson search combination in Summer 2011, CMS-NOTE-2011-005 (2011) [ATL-PHYS-PUB-2011-11] [INSPIRE].

[97] CRESST collaboration, Results on light dark matter particles with a low-threshold CRESST-II detector, Eur. Phys. J. C 76 (2016) 25 [arXiv:1509.01515] [InSPIRE].

[98] SuPERCDMS collaboration, New Results from the Search for Low-Mass Weakly Interacting Massive Particles with the CDMS Low Ionization Threshold Experiment, Phys. Rev. Lett. 116 (2016) 071301 [arXiv: 1509.02448] [INSPIRE].

[99] PandaX-II collaboration, Dark Matter Results From 54-Ton-Day Exposure of PandaX-II Experiment, Phys. Rev. Lett. 119 (2017) 181302 [arXiv:1708.06917] [INSPIRE]. 
[100] LUX collaboration, Results from a search for dark matter in the complete LUX exposure, Phys. Rev. Lett. 118 (2017) 021303 [arXiv: 1608.07648] [INSPIRE].

[101] XENON collaboration, First Dark Matter Search Results from the XENON1T Experiment, Phys. Rev. Lett. 119 (2017) 181301 [arXiv:1705.06655] [INSPIRE].

[102] CDEX collaboration, Limits on Light Weakly Interacting Massive Particles from the First $102.8 \mathrm{~kg} \times$ day Data of the CDEX-10 Experiment, Phys. Rev. Lett. 120 (2018) 241301 [arXiv: 1802.09016] [INSPIRE]. 


\section{The CMS collaboration}

Yerevan Physics Institute, Yerevan, Armenia

A.M. Sirunyan ${ }^{\dagger}$, A. Tumasyan

\section{Institut für Hochenergiephysik, Wien, Austria}

W. Adam, F. Ambrogi, T. Bergauer, J. Brandstetter, M. Dragicevic, J. Erö, A. Escalante Del Valle, M. Flechl, R. Frühwirth ${ }^{1}$, M. Jeitler ${ }^{1}$, N. Krammer, I. Krätschmer, D. Liko, T. Madlener, I. Mikulec, N. Rad, J. Schieck ${ }^{1}$, R. Schöfbeck, M. Spanring, D. Spitzbart, W. Waltenberger, J. Wittmann, C.-E. Wulz ${ }^{1}$, M. Zarucki

\section{Institute for Nuclear Problems, Minsk, Belarus}

V. Drugakov, V. Mossolov, J. Suarez Gonzalez

\section{Universiteit Antwerpen, Antwerpen, Belgium}

M.R. Darwish, E.A. De Wolf, D. Di Croce, X. Janssen, J. Lauwers, A. Lelek, M. Pieters, H. Rejeb Sfar, H. Van Haevermaet, P. Van Mechelen, S. Van Putte, N. Van Remortel

\section{Vrije Universiteit Brussel, Brussel, Belgium}

F. Blekman, E.S. Bols, S.S. Chhibra, J. D'Hondt, J. De Clercq, D. Lontkovskyi, S. Lowette, I. Marchesini, S. Moortgat, L. Moreels, Q. Python, K. Skovpen, S. Tavernier, W. Van Doninck, P. Van Mulders, I. Van Parijs

\section{Université Libre de Bruxelles, Bruxelles, Belgium}

D. Beghin, B. Bilin, H. Brun, B. Clerbaux, G. De Lentdecker, H. Delannoy, B. Dorney,

L. Favart, A. Grebenyuk, A.K. Kalsi, J. Luetic, A. Popov, N. Postiau, E. Starling,

L. Thomas, C. Vander Velde, P. Vanlaer, D. Vannerom, Q. Wang

\section{Ghent University, Ghent, Belgium}

T. Cornelis, D. Dobur, I. Khvastunov², C. Roskas, D. Trocino, M. Tytgat, W. Verbeke,

B. Vermassen, M. Vit, N. Zaganidis

Université Catholique de Louvain, Louvain-la-Neuve, Belgium

O. Bondu, G. Bruno, C. Caputo, P. David, C. Delaere, M. Delcourt, A. Giammanco, V. Lemaitre, A. Magitteri, J. Prisciandaro, A. Saggio, M. Vidal Marono, P. Vischia, J. Zobec

Centro Brasileiro de Pesquisas Fisicas, Rio de Janeiro, Brazil

F.L. Alves, G.A. Alves, G. Correia Silva, C. Hensel, A. Moraes, P. Rebello Teles

Universidade do Estado do Rio de Janeiro, Rio de Janeiro, Brazil

E. Belchior Batista Das Chagas, W. Carvalho, J. Chinellato ${ }^{3}$, E. Coelho, E.M. Da Costa, G.G. Da Silveira ${ }^{4}$, D. De Jesus Damiao, C. De Oliveira Martins, S. Fonseca De Souza, L.M. Huertas Guativa, H. Malbouisson, J. Martins ${ }^{5}$, D. Matos Figueiredo, M. Medina Jaime ${ }^{6}$, M. Melo De Almeida, C. Mora Herrera, L. Mundim, H. Nogima, W.L. Prado Da Silva, L.J. Sanchez Rosas, A. Santoro, A. Sznajder, M. Thiel, E.J. Tonelli Manganote ${ }^{3}$, F. Torres Da Silva De Araujo, A. Vilela Pereira 
Universidade Estadual Paulista ${ }^{a}$, Universidade Federal do $\mathrm{ABC}^{b}$, São Paulo, Brazil

S. Ahuja ${ }^{a}$, C.A. Bernardes ${ }^{a}$, L. Calligaris ${ }^{a}$, T.R. Fernandez Perez Tomei ${ }^{a}$, E.M. Gregores ${ }^{b}$, D.S. Lemos, P.G. Mercadante ${ }^{b}$, S.F. Novaes ${ }^{a}$, SandraS. Padula ${ }^{a}$

Institute for Nuclear Research and Nuclear Energy, Bulgarian Academy of Sciences, Sofia, Bulgaria

A. Aleksandrov, G. Antchev, R. Hadjiiska, P. Iaydjiev, A. Marinov, M. Misheva, M. Rodozov, M. Shopova, G. Sultanov

University of Sofia, Sofia, Bulgaria

M. Bonchev, A. Dimitrov, T. Ivanov, L. Litov, B. Pavlov, P. Petkov

Beihang University, Beijing, China

W. Fang $^{7}$, X. $\mathrm{Gao}^{7}$, L. Yuan

Institute of High Energy Physics, Beijing, China

M. Ahmad, G.M. Chen, H.S. Chen, M. Chen, C.H. Jiang, D. Leggat, H. Liao, Z. Liu, S.M. Shaheen ${ }^{8}$, A. Spiezia, J. Tao, E. Yazgan, H. Zhang, S. Zhang ${ }^{8}$, J. Zhao

State Key Laboratory of Nuclear Physics and Technology, Peking University, Beijing, China

A. Agapitos, Y. Ban, G. Chen, A. Levin, J. Li, L. Li, Q. Li, Y. Mao, S.J. Qian, D. Wang Tsinghua University, Beijing, China

Z. Hu, Y. Wang

Universidad de Los Andes, Bogota, Colombia

C. Avila, A. Cabrera, L.F. Chaparro Sierra, C. Florez, C.F. González Hernández, M.A. Segura Delgado

Universidad de Antioquia, Medellin, Colombia

J. Mejia Guisao, J.D. Ruiz Alvarez, C.A. Salazar González, N. Vanegas Arbelaez

University of Split, Faculty of Electrical Engineering, Mechanical Engineering and Naval Architecture, Split, Croatia

D. Giljanović, N. Godinovic, D. Lelas, I. Puljak, T. Sculac

University of Split, Faculty of Science, Split, Croatia

Z. Antunovic, M. Kovac

Institute Rudjer Boskovic, Zagreb, Croatia

V. Brigljevic, S. Ceci, D. Ferencek, K. Kadija, B. Mesic, M. Roguljic, A. Starodumov ${ }^{9}$, T. Susa

University of Cyprus, Nicosia, Cyprus

M.W. Ather, A. Attikis, E. Erodotou, A. Ioannou, M. Kolosova, S. Konstantinou, G. Mavromanolakis, J. Mousa, C. Nicolaou, F. Ptochos, P.A. Razis, H. Rykaczewski, D. Tsiakkouri 
Charles University, Prague, Czech Republic

M. Finger ${ }^{10}$, M. Finger Jr. ${ }^{10}$, A. Kveton, J. Tomsa

Escuela Politecnica Nacional, Quito, Ecuador

E. Ayala

Universidad San Francisco de Quito, Quito, Ecuador

E. Carrera Jarrin

Academy of Scientific Research and Technology of the Arab Republic of Egypt, Egyptian Network of High Energy Physics, Cairo, Egypt

S. Elgammal ${ }^{11}$, A. Ellithi Kamel ${ }^{12}$

National Institute of Chemical Physics and Biophysics, Tallinn, Estonia

S. Bhowmik, A. Carvalho Antunes De Oliveira, R.K. Dewanjee, K. Ehataht, M. Kadastik, M. Raidal, C. Veelken

Department of Physics, University of Helsinki, Helsinki, Finland

P. Eerola, L. Forthomme, H. Kirschenmann, K. Osterberg, M. Voutilainen

Helsinki Institute of Physics, Helsinki, Finland

F. Garcia, J. Havukainen, J.K. Heikkilä, T. Järvinen, V. Karimäki, R. Kinnunen,

T. Lampén, K. Lassila-Perini, S. Laurila, S. Lehti, T. Lindén, P. Luukka, T. Mäenpää,

H. Siikonen, E. Tuominen, J. Tuominiemi

Lappeenranta University of Technology, Lappeenranta, Finland

T. Tuuva

IRFU, CEA, Université Paris-Saclay, Gif-sur-Yvette, France

M. Besancon, F. Couderc, M. Dejardin, D. Denegri, B. Fabbro, J.L. Faure, F. Ferri, S. Ganjour, A. Givernaud, P. Gras, G. Hamel de Monchenault, P. Jarry, C. Leloup, E. Locci, J. Malcles, J. Rander, A. Rosowsky, M.Ö. Sahin, A. Savoy-Navarro ${ }^{13}$, M. Titov

Laboratoire Leprince-Ringuet, CNRS/IN2P3, Ecole Polytechnique, Institut Polytechnique de Paris

C. Amendola, F. Beaudette, P. Busson, C. Charlot, B. Diab, R. Granier de Cassagnac, I. Kucher, A. Lobanov, C. Martin Perez, M. Nguyen, C. Ochando, P. Paganini, J. Rembser, R. Salerno, J.B. Sauvan, Y. Sirois, A. Zabi, A. Zghiche

Université de Strasbourg, CNRS, IPHC UMR 7178, Strasbourg, France

J.-L. Agram ${ }^{14}$, J. Andrea, D. Bloch, G. Bourgatte, J.-M. Brom, E.C. Chabert, C. Collard, E. Conte ${ }^{14}$, J.-C. Fontaine ${ }^{14}$, D. Gelé, U. Goerlach, M. Jansová, A.-C. Le Bihan, N. Tonon, P. Van Hove

Centre de Calcul de l'Institut National de Physique Nucleaire et de Physique des Particules, CNRS/IN2P3, Villeurbanne, France

S. Gadrat 
Université de Lyon, Université Claude Bernard Lyon 1, CNRS-IN2P3, Institut de Physique Nucléaire de Lyon, Villeurbanne, France

S. Beauceron, C. Bernet, G. Boudoul, C. Camen, N. Chanon, R. Chierici, D. Contardo, P. Depasse, H. El Mamouni, J. Fay, S. Gascon, M. Gouzevitch, B. Ille, Sa. Jain, F. Lagarde, I.B. Laktineh, H. Lattaud, M. Lethuillier, L. Mirabito, S. Perries, V. Sordini, G. Touquet, M. Vander Donckt, S. Viret

Georgian Technical University, Tbilisi, Georgia

A. Khvedelidze ${ }^{10}$

Tbilisi State University, Tbilisi, Georgia

Z. Tsamalaidze ${ }^{10}$

RWTH Aachen University, I. Physikalisches Institut, Aachen, Germany

C. Autermann, L. Feld, M.K. Kiesel, K. Klein, M. Lipinski, D. Meuser, A. Pauls, M. Preuten, M.P. Rauch, C. Schomakers, J. Schulz, M. Teroerde, B. Wittmer

RWTH Aachen University, III. Physikalisches Institut A, Aachen, Germany

A. Albert, M. Erdmann, S. Erdweg, T. Esch, B. Fischer, R. Fischer, S. Ghosh, T. Hebbeker, K. Hoepfner, H. Keller, L. Mastrolorenzo, M. Merschmeyer, A. Meyer, P. Millet, G. Mocellin, S. Mondal, S. Mukherjee, D. Noll, A. Novak, T. Pook, A. Pozdnyakov, T. Quast, M. Radziej, Y. Rath, H. Reithler, M. Rieger, A. Schmidt, S.C. Schuler, A. Sharma, S. Thüer, S. Wiedenbeck

RWTH Aachen University, III. Physikalisches Institut B, Aachen, Germany G. Flügge, W. Haj Ahmad ${ }^{15}$, O. Hlushchenko, T. Kress, T. Müller, A. Nehrkorn, A. Nowack, C. Pistone, O. Pooth, D. Roy, H. Sert, A. Stahl ${ }^{16}$

Deutsches Elektronen-Synchrotron, Hamburg, Germany

M. Aldaya Martin, C. Asawatangtrakuldee, P. Asmuss, I. Babounikau, H. Bakhshiansohi, K. Beernaert, O. Behnke, U. Behrens, A. Bermúdez Martínez, D. Bertsche, A.A. Bin Anuar, K. Borras ${ }^{17}$, V. Botta, A. Campbell, A. Cardini, P. Connor, S. Consuegra Rodríguez, C. Contreras-Campana, V. Danilov, A. De Wit, M.M. Defranchis, C. Diez Pardos, D. Domínguez Damiani, G. Eckerlin, D. Eckstein, T. Eichhorn, A. Elwood, E. Eren, E. Gallo ${ }^{18}$, A. Geiser, J.M. Grados Luyando, A. Grohsjean, M. Guthoff, M. Haranko, A. Harb, A. Jafari, N.Z. Jomhari, H. Jung, A. Kasem ${ }^{17}$, M. Kasemann, H. Kaveh, J. Keaveney, C. Kleinwort, J. Knolle, D. Krücker, W. Lange, T. Lenz, J. Leonard, J. Lidrych, K. Lipka, W. Lohmann ${ }^{19}$, R. Mankel, I.-A. Melzer-Pellmann, A.B. Meyer, M. Meyer, M. Missiroli, G. Mittag, J. Mnich, A. Mussgiller, V. Myronenko, D. Pérez Adán, S.K. Pflitsch, D. Pitzl, A. Raspereza, A. Saibel, M. Savitskyi, V. Scheurer, P. Schütze, C. Schwanenberger, R. Shevchenko, A. Singh, H. Tholen, O. Turkot, A. Vagnerini, M. Van De Klundert, G.P. Van Onsem, R. Walsh, Y. Wen, K. Wichmann, C. Wissing, O. Zenaiev, R. Zlebcik

\section{University of Hamburg, Hamburg, Germany}

R. Aggleton, S. Bein, L. Benato, A. Benecke, V. Blobel, T. Dreyer, A. Ebrahimi, A. Fröhlich, C. Garbers, E. Garutti, D. Gonzalez, P. Gunnellini, J. Haller, A. Hinzmann, 
A. Karavdina, G. Kasieczka, R. Klanner, R. Kogler, N. Kovalchuk, S. Kurz, V. Kutzner, J. Lange, T. Lange, A. Malara, D. Marconi, J. Multhaup, M. Niedziela, C.E.N. Niemeyer, D. Nowatschin, A. Perieanu, A. Reimers, O. Rieger, C. Scharf, P. Schleper, S. Schumann, J. Schwandt, J. Sonneveld, H. Stadie, G. Steinbrück, F.M. Stober, M. Stöver, B. Vormwald, I. Zoi

Karlsruher Institut fuer Technologie, Karlsruhe, Germany

M. Akbiyik, C. Barth, M. Baselga, S. Baur, T. Berger, E. Butz, R. Caspart, T. Chwalek, W. De Boer, A. Dierlamm, K. El Morabit, N. Faltermann, M. Giffels, P. Goldenzweig, A. Gottmann, M.A. Harrendorf, F. Hartmann ${ }^{16}$, U. Husemann, S. Kudella, S. Mitra, M.U. Mozer, Th. Müller, M. Musich, A. Nürnberg, G. Quast, K. Rabbertz, M. Schröder, I. Shvetsov, H.J. Simonis, R. Ulrich, M. Weber, C. Wöhrmann, R. Wolf

Institute of Nuclear and Particle Physics (INPP), NCSR Demokritos, Aghia Paraskevi, Greece

G. Anagnostou, P. Asenov, G. Daskalakis, T. Geralis, A. Kyriakis, D. Loukas, G. Paspalaki

National and Kapodistrian University of Athens, Athens, Greece

M. Diamantopoulou, G. Karathanasis, P. Kontaxakis, A. Panagiotou, I. Papavergou, N. Saoulidou, A. Stakia, K. Theofilatos, K. Vellidis

National Technical University of Athens, Athens, Greece

G. Bakas, K. Kousouris, I. Papakrivopoulos, G. Tsipolitis

University of Ioánnina, Ioánnina, Greece

I. Evangelou, C. Foudas, P. Gianneios, P. Katsoulis, P. Kokkas, S. Mallios, K. Manitara, N. Manthos, I. Papadopoulos, J. Strologas, F.A. Triantis, D. Tsitsonis

MTA-ELTE Lendület CMS Particle and Nuclear Physics Group, Eötvös Loránd University, Budapest, Hungary

M. Bartók ${ }^{20}$, M. Csanad, P. Major, K. Mandal, A. Mehta, M.I. Nagy, G. Pasztor, O. Surányi, G.I. Veres

Wigner Research Centre for Physics, Budapest, Hungary

G. Bencze, C. Hajdu, D. Horvath ${ }^{21}$, F. Sikler, T.Á. Vámi, V. Veszpremi, G. Vesztergombi ${ }^{\dagger}$

Institute of Nuclear Research ATOMKI, Debrecen, Hungary

N. Beni, S. Czellar, J. Karancsi ${ }^{20}$, A. Makovec, J. Molnar, Z. Szillasi

Institute of Physics, University of Debrecen, Debrecen, Hungary

P. Raics, D. Teyssier, Z.L. Trocsanyi, B. Ujvari

Eszterhazy Karoly University, Karoly Robert Campus, Gyongyos, Hungary

T.F. Csorgo, W.J. Metzger, F. Nemes, T. Novak

Indian Institute of Science (IISc), Bangalore, India

S. Choudhury, J.R. Komaragiri, P.C. Tiwari 
National Institute of Science Education and Research, HBNI, Bhubaneswar, India

S. Bahinipati ${ }^{23}$, C. Kar, G. Kole, P. Mal, V.K. Muraleedharan Nair Bindhu, A. Nayak ${ }^{24}$, D.K. Sahoo ${ }^{23}$, S.K. Swain

Panjab University, Chandigarh, India

S. Bansal, S.B. Beri, V. Bhatnagar, S. Chauhan, R. Chawla, N. Dhingra, R. Gupta, A. Kaur, M. Kaur, S. Kaur, P. Kumari, M. Lohan, M. Meena, K. Sandeep, S. Sharma, J.B. Singh

University of Delhi, Delhi, India

A. Bhardwaj, B.C. Choudhary, R.B. Garg, M. Gola, S. Keshri, Ashok Kumar, S. Malhotra, M. Naimuddin, P. Priyanka, K. Ranjan, Aashaq Shah, R. Sharma

Saha Institute of Nuclear Physics, HBNI, Kolkata, India

R. Bhardwaj ${ }^{25}$, M. Bharti ${ }^{25}$, R. Bhattacharya, S. Bhattacharya, U. Bhawandeep ${ }^{25}$, D. Bhowmik, S. Dey, S. Dutta, S. Ghosh, M. Maity ${ }^{26}$, K. Mondal, S. Nandan, A. Purohit, P.K. Rout, A. Roy, G. Saha, S. Sarkar, T. $\operatorname{Sarkar}^{26}$, M. Sharan, B. Singh ${ }^{25}$, S. Thakur ${ }^{25}$

Indian Institute of Technology Madras, Madras, India

P.K. Behera, P. Kalbhor, A. Muhammad, P.R. Pujahari, A. Sharma, A.K. Sikdar

Bhabha Atomic Research Centre, Mumbai, India

R. Chudasama, D. Dutta, V. Jha, V. Kumar, D.K. Mishra, P.K. Netrakanti, L.M. Pant, P. Shukla

Tata Institute of Fundamental Research-A, Mumbai, India

T. Aziz, M.A. Bhat, S. Dugad, G.B. Mohanty, N. Sur, RavindraKumar Verma

Tata Institute of Fundamental Research-B, Mumbai, India

S. Banerjee, S. Bhattacharya, S. Chatterjee, P. Das, M. Guchait, S. Karmakar, S. Kumar, G. Majumder, K. Mazumdar, N. Sahoo, S. Sawant

Indian Institute of Science Education and Research (IISER), Pune, India

S. Chauhan, S. Dube, V. Hegde, A. Kapoor, K. Kothekar, S. Pandey, A. Rane, A. Rastogi, S. Sharma

Institute for Research in Fundamental Sciences (IPM), Tehran, Iran

S. Chenarani ${ }^{27}$, E. Eskandari Tadavani, S.M. Etesami ${ }^{27}$, M. Khakzad, M. Mohammadi Najafabadi, M. Naseri, F. Rezaei Hosseinabadi

University College Dublin, Dublin, Ireland

M. Felcini, M. Grunewald

INFN Sezione di Bari ${ }^{a}$, Università di Bari ${ }^{b}$, Politecnico di Bari ${ }^{c}$, Bari, Italy M. Abbrescia ${ }^{a, b}$, R. Aly ${ }^{a, b}$, C. Calabria ${ }^{a, b}$, A. Colaleo ${ }^{a}$, D. Creanza ${ }^{a, c}$, L. Cristella ${ }^{a, b}$, N. De Filippis ${ }^{a, c}$, M. De Palma ${ }^{a, b}$, A. Di Florio ${ }^{a, b}$, L. Fiore $^{a}$, A. Gelmi $^{a, b}$, G. Iaselli $^{a, c}$, M. Ince ${ }^{a, b}$, S. Lezki ${ }^{a, b}$, G. Maggi ${ }^{a, c}$, M. Maggi ${ }^{a}$, G. Miniello ${ }^{a, b}$, S. My ${ }^{a, b}$, S. Nuzzo $^{a, b}$, 


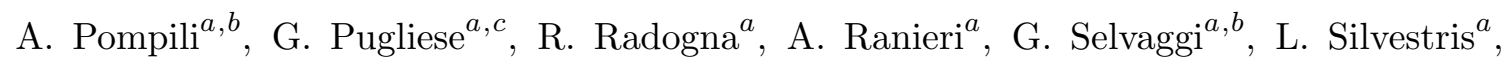
R. Venditti ${ }^{a}$, P. Verwilligen ${ }^{a}$

INFN Sezione di Bologna ${ }^{a}$, Università di Bologna ${ }^{b}$, Bologna, Italy

G. Abbiendi ${ }^{a}$, C. Battilana ${ }^{a, b}$, D. Bonacorsi ${ }^{a, b}$, L. Borgonovi $^{a, b}$, S. Braibant-Giacomelli $^{a, b}$, R. Campanini ${ }^{a, b}$, P. Capiluppi ${ }^{a, b}$, A. Castro ${ }^{a, b}$, F.R. Cavallo ${ }^{a}$, C. Ciocca $^{a}$, G. Codispoti $^{a, b}$, M. Cuffiani ${ }^{a, b}$, G.M. Dallavalle ${ }^{a}$, F. Fabbri ${ }^{a}$, A. Fanfani ${ }^{a, b}$, E. Fontanesi, P. Giacomelli ${ }^{a}$, C. Grandi ${ }^{a}$, L. Guiducci ${ }^{a, b}$, F. Iemmi ${ }^{a, b}$, S. Lo Meo ${ }^{a, 28}$, S. Marcellini ${ }^{a}$, G. Masetti ${ }^{a}$,

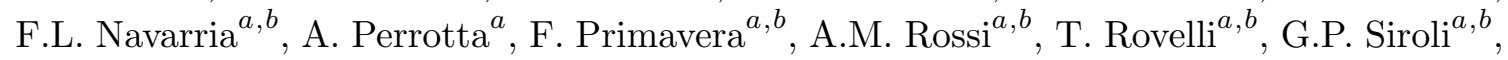
N. Tosi ${ }^{a}$

INFN Sezione di Catania ${ }^{a}$, Università di Catania $^{b}$, Catania, Italy

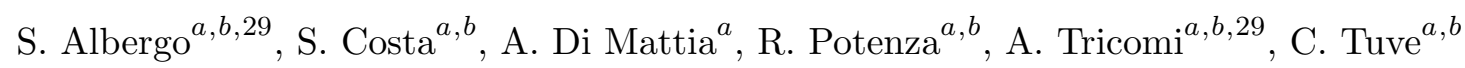

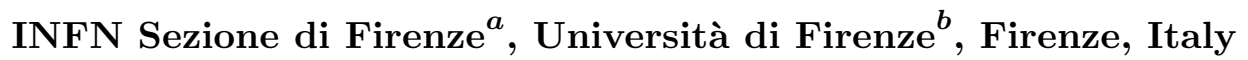

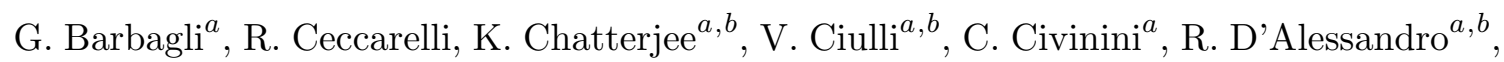

E. Focardi ${ }^{a, b}$, G. Latino, P. Lenzi ${ }^{a, b}$, M. Meschini ${ }^{a}$, S. Paoletti $^{a}$, G. Sguazzoni $^{a}$, D. Strom ${ }^{a}$, L. Viliani ${ }^{a}$

INFN Laboratori Nazionali di Frascati, Frascati, Italy

L. Benussi, S. Bianco, D. Piccolo

INFN Sezione di Genova ${ }^{a}$, Università di Genova ${ }^{b}$, Genova, Italy

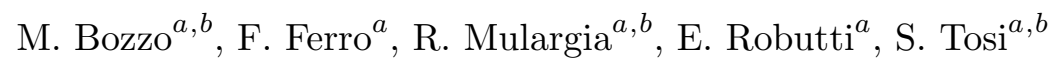

INFN Sezione di Milano-Bicocca ${ }^{a}$, Università di Milano-Bicocca ${ }^{b}$, Milano, Italy

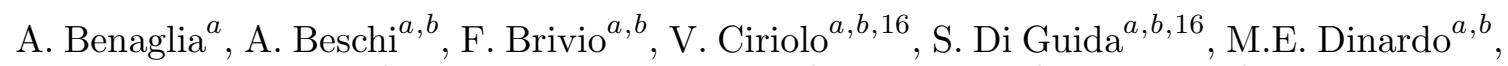

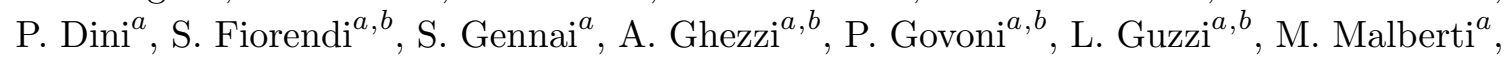

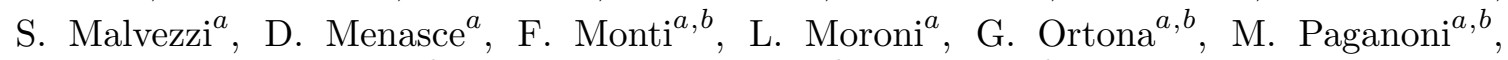
D. Pedrini ${ }^{a}$, S. Ragazzi ${ }^{a, b}$, T. Tabarelli de Fatis ${ }^{a, b}$, D. Zuolo ${ }^{a, b}$

INFN Sezione di Napoli ${ }^{a}$, Università di Napoli 'Federico II'b , Napoli, Italy, Università della Basilicata $^{c}$, Potenza, Italy, Università G. Marconi ${ }^{d}$, Roma, Italy

S. Buontempo $^{a}$, N. Cavallo ${ }^{a, c}$, A. De Iorio ${ }^{a, b}$, A. Di Crescenzo ${ }^{a, b}$, F. Fabozzi $^{a, c}$, F. Fienga $^{a}$, G. Galati ${ }^{a}$, A.O.M. Iorio ${ }^{a, b}$, L. Lista ${ }^{a, b}$, S. Meola ${ }^{a, d, 16}$, P. Paolucci ${ }^{a, 16}$, B. Rossi ${ }^{a}$, C. Sciacca $^{a, b}$, E. Voevodina ${ }^{a, b}$

INFN Sezione di Padova ${ }^{a}$, Università di Padova ${ }^{b}$, Padova, Italy, Università di Trento $^{c}$, Trento, Italy

P. Azzi ${ }^{a}$, N. Bacchetta ${ }^{a}$, D. Bisello ${ }^{a, b}$, A. Boletti ${ }^{a, b}$, A. Bragagnolo, R. Carlin ${ }^{a, b}$, P. Checchia ${ }^{a}$, P. De Castro Manzano ${ }^{a}$, T. Dorigo ${ }^{a}$, U. Dosselli ${ }^{a}$, F. Gasparini ${ }^{a, b}$, U. Gasparini $^{a, b}$, A. Gozzelino ${ }^{a}$, S.Y. Hoh, P. Lujan, M. Margoni ${ }^{a, b}$, A.T. Meneguzzo $^{a, b}$, J. Pazzini ${ }^{a, b}$, M. Presilla ${ }^{b}$, P. Ronchese ${ }^{a, b}$, R. Rossin ${ }^{a, b}$, F. Simonetto ${ }^{a, b}$, A. Tiko, M. Tosi ${ }^{a, b}$, M. Zanetti ${ }^{a, b}$, P. Zotto ${ }^{a, b}$, G. Zumerle ${ }^{a, b}$ 
INFN Sezione di Pavia ${ }^{a}$, Università di Pavia ${ }^{b}$, Pavia, Italy

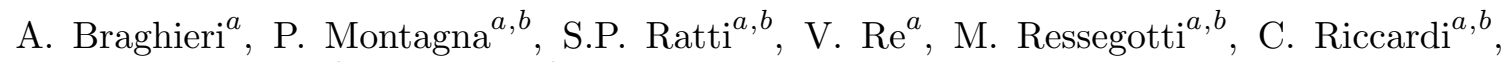
P. Salvini ${ }^{a}$, I. Vai ${ }^{a, b}$, P. Vitulo ${ }^{a, b}$

INFN Sezione di Perugia ${ }^{a}$, Università di Perugia ${ }^{b}$, Perugia, Italy

M. Biasini ${ }^{a, b}$, G.M. Bilei ${ }^{a}$, C. Cecchi ${ }^{a, b}$, D. Ciangottini ${ }^{a, b}$, L. Fanò ${ }^{a, b}$, P. Lariccia $^{a, b}$, R. Leonardi ${ }^{a, b}$, E. Manoni ${ }^{a}$, G. Mantovani ${ }^{a, b}$, V. Mariani ${ }^{a, b}$, M. Menichelli $^{a}$, A. Rossi $^{a, b}$, A. Santocchia ${ }^{a, b}$, D. Spiga ${ }^{a}$

INFN Sezione di Pisa ${ }^{a}$, Università di Pisa ${ }^{b}$, Scuola Normale Superiore di Pisa ${ }^{c}$, Pisa, Italy

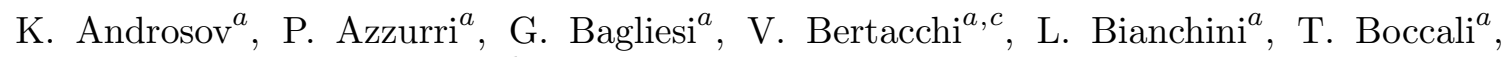

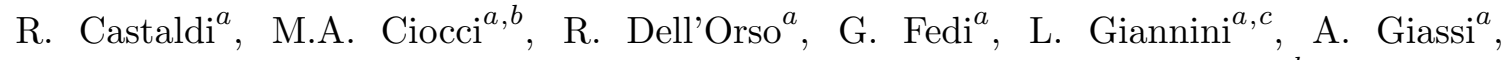

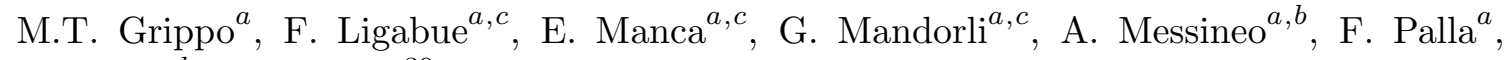
A. Rizzi ${ }^{a}, b$, G. Rolandi ${ }^{30}$, S. Roy Chowdhury, A. Scribano ${ }^{a}$, P. Spagnolo $^{a}$, R. Tenchini ${ }^{a}$, G. Tonelli ${ }^{a, b}$, N. Turini, A. Venturi ${ }^{a}$, P.G. Verdini ${ }^{a}$

INFN Sezione di Roma ${ }^{a}$, Sapienza Università di Roma ${ }^{b}$, Rome, Italy

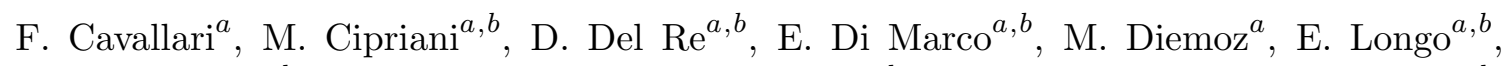
B. Marzocchi $^{a, b}$, P. Meridiani ${ }^{a}$, G. Organtini ${ }^{a, b}$, F. Pandolfi ${ }^{a}$, R. Paramatti ${ }^{a, b}$,

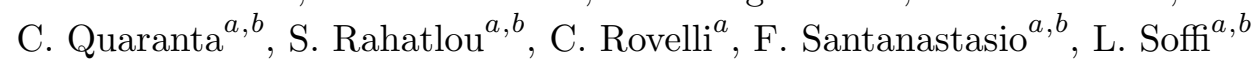

INFN Sezione di Torino ${ }^{a}$, Università di Torino ${ }^{b}$, Torino, Italy, Università del Piemonte Orientale ${ }^{c}$, Novara, Italy

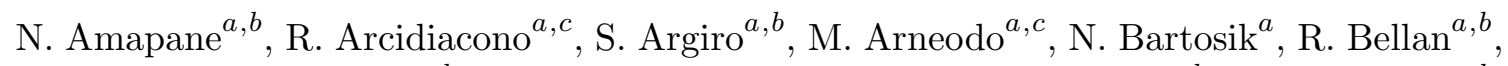

C. Biino ${ }^{a}$, A. Cappati ${ }^{a, b}$, N. Cartiglia ${ }^{a}$, S. Cometti ${ }^{a}$, M. Costa $^{a, b}$, R. Covarelli ${ }^{a, b}$,

N. $\operatorname{Demaria}^{a}$, B. Kiani ${ }^{a, b}$, C. Mariotti $^{a}$, S. Maselli ${ }^{a}$, E. Migliore ${ }^{a, b}$, V. Monaco ${ }^{a, b}$,

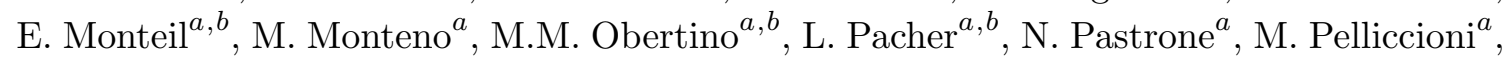
G.L. Pinna Angioni ${ }^{a, b}$, A. Romero ${ }^{a, b}$, M. Ruspa ${ }^{a, c}$, R. Sacchi ${ }^{a, b}$, R. Salvatico ${ }^{a, b}$, K. Shchelina ${ }^{a, b}$, V. Sola ${ }^{a}$, A. Solano ${ }^{a, b}$, D. Soldi ${ }^{a, b}$, A. Staiano ${ }^{a}$

INFN Sezione di Trieste ${ }^{a}$, Università di Trieste ${ }^{b}$, Trieste, Italy

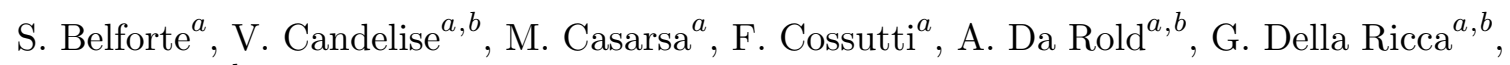
F. Vazzoler $^{a, b}$, A. Zanetti ${ }^{a}$

Kyungpook National University, Daegu, Korea

B. Kim, D.H. Kim, G.N. Kim, M.S. Kim, J. Lee, S.W. Lee, C.S. Moon, Y.D. Oh, S.I. Pak, S. Sekmen, D.C. Son, Y.C. Yang

Chonnam National University, Institute for Universe and Elementary Particles, Kwangju, Korea

H. Kim, D.H. Moon, G. Oh

Hanyang University, Seoul, Korea

B. Francois, T.J. Kim, J. Park 
Korea University, Seoul, Korea

S. Cho, S. Choi, Y. Go, D. Gyun, S. Ha, B. Hong, K. Lee, K.S. Lee, J. Lim, J. Park, S.K. Park, Y. Roh

Kyung Hee University, Department of Physics

J. Goh

Sejong University, Seoul, Korea

H.S. Kim

Seoul National University, Seoul, Korea

J. Almond, J.H. Bhyun, J. Choi, S. Jeon, J. Kim, J.S. Kim, H. Lee, K. Lee, S. Lee, K. Nam, S.B. Oh, B.C. Radburn-Smith, S.h. Seo, U.K. Yang, H.D. Yoo, I. Yoon, G.B. Yu

University of Seoul, Seoul, Korea

D. Jeon, H. Kim, J.H. Kim, J.S.H. Lee, I.C. Park, I. Watson

Sungkyunkwan University, Suwon, Korea

Y. Choi, C. Hwang, Y. Jeong, J. Lee, Y. Lee, I. Yu

Riga Technical University, Riga, Latvia

V. Veckalns ${ }^{31}$

Vilnius University, Vilnius, Lithuania

V. Dudenas, A. Juodagalvis, J. Vaitkus

National Centre for Particle Physics, Universiti Malaya, Kuala Lumpur, Malaysia

Z.A. Ibrahim, F. Mohamad Idris ${ }^{32}$, W.A.T. Wan Abdullah, M.N. Yusli, Z. Zolkapli

Universidad de Sonora (UNISON), Hermosillo, Mexico

J.F. Benitez, A. Castaneda Hernandez, J.A. Murillo Quijada, L. Valencia Palomo

Centro de Investigacion y de Estudios Avanzados del IPN, Mexico City, Mexico

H. Castilla-Valdez, E. De La Cruz-Burelo, I. Heredia-De La Cruz ${ }^{33}$, R. Lopez-Fernandez,

A. Sanchez-Hernandez

Universidad Iberoamericana, Mexico City, Mexico

S. Carrillo Moreno, C. Oropeza Barrera, M. Ramirez-Garcia, F. Vazquez Valencia

Benemerita Universidad Autonoma de Puebla, Puebla, Mexico

J. Eysermans, I. Pedraza, H.A. Salazar Ibarguen, C. Uribe Estrada

Universidad Autónoma de San Luis Potosí, San Luis Potosí, Mexico

A. Morelos Pineda

University of Montenegro, Podgorica, Montenegro

N. Raicevic

University of Auckland, Auckland, New Zealand

D. Krofcheck 
University of Canterbury, Christchurch, New Zealand

S. Bheesette, P.H. Butler

National Centre for Physics, Quaid-I-Azam University, Islamabad, Pakistan

A. Ahmad, M. Ahmad, Q. Hassan, H.R. Hoorani, W.A. Khan, S. Qazi, M.A. Shah, M. Waqas

AGH University of Science and Technology Faculty of Computer Science, Electronics and Telecommunications, Krakow, Poland

V. Avati, L. Grzanka, M. Malawski

National Centre for Nuclear Research, Swierk, Poland

H. Bialkowska, M. Bluj, B. Boimska, M. Górski, M. Kazana, M. Szleper, P. Zalewski

Institute of Experimental Physics, Faculty of Physics, University of Warsaw, Warsaw, Poland

K. Bunkowski, A. Byszuk ${ }^{34}$, K. Doroba, A. Kalinowski, M. Konecki, J. Krolikowski, M. Misiura, M. Olszewski, A. Pyskir, M. Walczak

Laboratório de Instrumentação e Física Experimental de Partículas, Lisboa, Portugal

M. Araujo, P. Bargassa, D. Bastos, A. Di Francesco, P. Faccioli, B. Galinhas, M. Gallinaro, J. Hollar, N. Leonardo, J. Seixas, G. Strong, O. Toldaiev, J. Varela

Joint Institute for Nuclear Research, Dubna, Russia

V. Alexakhin, P. Bunin, Y. Ershov, I. Golutvin, N. Gorbounov, A. Kamenev, V. Karjavine, V. Korenkov, A. Lanev, A. Malakhov, V. Matveev ${ }^{35,36}$, P. Moisenz, V. Palichik, V. Perelygin, M. Savina, S. Shmatov, S. Shulha, N. Voytishin, B.S. Yuldashev ${ }^{37}$, A. Zarubin

Petersburg Nuclear Physics Institute, Gatchina (St. Petersburg), Russia

L. Chtchipounov, V. Golovtsov, Y. Ivanov, V. Kim ${ }^{38}$, E. Kuznetsova ${ }^{39}$, P. Levchenko, V. Murzin, V. Oreshkin, I. Smirnov, D. Sosnov, V. Sulimov, L. Uvarov, A. Vorobyev

Institute for Nuclear Research, Moscow, Russia

Yu. Andreev, A. Dermenev, S. Gninenko, N. Golubev, A. Karneyeu, M. Kirsanov, N. Krasnikov, A. Pashenkov, D. Tlisov, A. Toropin

Institute for Theoretical and Experimental Physics named by A.I. Alikhanov of NRC 'Kurchatov Institute', Moscow, Russia

V. Epshteyn, V. Gavrilov, N. Lychkovskaya, A. Nikitenko ${ }^{40}$, V. Popov, I. Pozdnyakov,

G. Safronov, A. Spiridonov, A. Stepennov, M. Toms, E. Vlasov, A. Zhokin

Moscow Institute of Physics and Technology, Moscow, Russia

T. Aushev

National Research Nuclear University 'Moscow Engineering Physics Institute' (MEPhI), Moscow, Russia

M. Chadeeva ${ }^{41}$, P. Parygin, D. Philippov, E. Popova, V. Rusinov 
P.N. Lebedev Physical Institute, Moscow, Russia

V. Andreev, M. Azarkin, I. Dremin, M. Kirakosyan, A. Terkulov

Skobeltsyn Institute of Nuclear Physics, Lomonosov Moscow State University, Moscow, Russia

A. Baskakov, A. Belyaev, E. Boos, V. Bunichev, M. Dubinin ${ }^{42}$, L. Dudko, A. Ershov,

A. Gribushin, V. Klyukhin, O. Kodolova, I. Lokhtin, S. Obraztsov, V. Savrin

Novosibirsk State University (NSU), Novosibirsk, Russia

A. Barnyakov ${ }^{43}$, V. Blinov ${ }^{43}$, T. Dimova ${ }^{43}$, L. Kardapoltsev ${ }^{43}$, Y. Skovpen ${ }^{43}$

Institute for High Energy Physics of National Research Centre 'Kurchatov Institute', Protvino, Russia

I. Azhgirey, I. Bayshev, S. Bitioukov, V. Kachanov, D. Konstantinov, P. Mandrik,

V. Petrov, R. Ryutin, S. Slabospitskii, A. Sobol, S. Troshin, N. Tyurin, A. Uzunian,

A. Volkov

National Research Tomsk Polytechnic University, Tomsk, Russia

A. Babaev, A. Iuzhakov, V. Okhotnikov

Tomsk State University, Tomsk, Russia

V. Borchsh, V. Ivanchenko, E. Tcherniaev

University of Belgrade: Faculty of Physics and VINCA Institute of Nuclear Sciences

P. Adzic ${ }^{44}$, P. Cirkovic, D. Devetak, M. Dordevic, P. Milenovic, J. Milosevic, M. Stojanovic

Centro de Investigaciones Energéticas Medioambientales y Tecnológicas (CIEMAT), Madrid, Spain

M. Aguilar-Benitez, J. Alcaraz Maestre, A. Álvarez Fernández, I. Bachiller, M. Barrio Luna, J.A. Brochero Cifuentes, C.A. Carrillo Montoya, M. Cepeda, M. Cerrada, N. Colino, B. De La Cruz, A. Delgado Peris, C. Fernandez Bedoya, J.P. Fernández Ramos, J. Flix, M.C. Fouz, O. Gonzalez Lopez, S. Goy Lopez, J.M. Hernandez, M.I. Josa, D. Moran, Á. Navarro Tobar, A. Pérez-Calero Yzquierdo, J. Puerta Pelayo, I. Redondo, L. Romero, S. Sánchez Navas, M.S. Soares, A. Triossi, C. Willmott

Universidad Autónoma de Madrid, Madrid, Spain

C. Albajar, J.F. de Trocóniz

Universidad de Oviedo, Instituto Universitario de Ciencias y Tecnologías Espaciales de Asturias (ICTEA), Oviedo, Spain

B. Alvarez Gonzalez, J. Cuevas, C. Erice, J. Fernandez Menendez, S. Folgueras, I. Gonzalez Caballero, J.R. González Fernández, E. Palencia Cortezon, V. Rodríguez Bouza, S. Sanchez Cruz

Instituto de Física de Cantabria (IFCA), CSIC-Universidad de Cantabria, Santander, Spain

I.J. Cabrillo, A. Calderon, B. Chazin Quero, J. Duarte Campderros, M. Fernandez, P.J. Fernández Manteca, A. García Alonso, G. Gomez, C. Martinez Rivero, P. Mar- 
tinez Ruiz del Arbol, F. Matorras, J. Piedra Gomez, C. Prieels, T. Rodrigo, A. Ruiz-Jimeno, L. Russo $^{45}$, L. Scodellaro, N. Trevisani, I. Vila, J.M. Vizan Garcia

\section{University of Colombo, Colombo, Sri Lanka}

K. Malagalage

\section{University of Ruhuna, Department of Physics, Matara, Sri Lanka}

W.G.D. Dharmaratna, N. Wickramage

\section{CERN, European Organization for Nuclear Research, Geneva, Switzerland}

D. Abbaneo, B. Akgun, E. Auffray, G. Auzinger, J. Baechler, P. Baillon, A.H. Ball, D. Barney, J. Bendavid, M. Bianco, A. Bocci, E. Bossini, C. Botta, E. Brondolin, T. Camporesi, A. Caratelli, G. Cerminara, E. Chapon, G. Cucciati, D. d'Enterria, A. Dabrowski, N. Daci, V. Daponte, A. David, O. Davignon, A. De Roeck, N. Deelen, M. Deile, M. Dobson, M. Dünser, N. Dupont, A. Elliott-Peisert, F. Fallavollita ${ }^{46}$, D. Fasanella, G. Franzoni, J. Fulcher, W. Funk, S. Giani, D. Gigi, A. Gilbert, K. Gill, F. Glege, M. Gruchala, M. Guilbaud, D. Gulhan, J. Hegeman, C. Heidegger, Y. Iiyama, V. Innocente, P. Janot, O. Karacheban ${ }^{19}$, J. Kaspar, J. Kieseler, M. Krammer ${ }^{1}$, C. Lange, P. Lecoq, C. Lourenço, L. Malgeri, M. Mannelli, A. Massironi, F. Meijers, J.A. Merlin, S. Mersi, E. Meschi, F. Moortgat, M. Mulders, J. Ngadiuba, S. Nourbakhsh, S. Orfanelli, L. Orsini, F. Pantaleo ${ }^{16}$, L. Pape, E. Perez, M. Peruzzi, A. Petrilli, G. Petrucciani, A. Pfeiffer, M. Pierini, F.M. Pitters, M. Quinto, D. Rabady, A. Racz, M. Rovere, H. Sakulin, C. Schäfer, C. Schwick, M. Selvaggi, A. Sharma, P. Silva, W. Snoeys, P. Sphicas ${ }^{47}$, J. Steggemann, V.R. Tavolaro, D. Treille, A. Tsirou, A. Vartak, M. Verzetti, W.D. Zeuner

\section{Paul Scherrer Institut, Villigen, Switzerland}

L. Caminada ${ }^{48}$, K. Deiters, W. Erdmann, R. Horisberger, Q. Ingram, H.C. Kaestli, D. Kotlinski, U. Langenegger, T. Rohe, S.A. Wiederkehr

ETH Zurich - Institute for Particle Physics and Astrophysics (IPA), Zurich, Switzerland

M. Backhaus, P. Berger, N. Chernyavskaya, G. Dissertori, M. Dittmar, M. Donegà, C. Dorfer, T.A. Gómez Espinosa, C. Grab, D. Hits, T. Klijnsma, W. Lustermann, R.A. Manzoni, M. Marionneau, M.T. Meinhard, F. Micheli, P. Musella, F. Nessi-Tedaldi, F. Pauss, G. Perrin, L. Perrozzi, S. Pigazzini, M. Reichmann, C. Reissel, T. Reitenspiess, D. Ruini, D.A. Sanz Becerra, M. Schönenberger, L. Shchutska, M.L. Vesterbacka Olsson, R. Wallny, D.H. Zhu

\section{Universität Zürich, Zurich, Switzerland}

T.K. Aarrestad, C. Amsler ${ }^{49}$, D. Brzhechko, M.F. Canelli, A. De Cosa, R. Del Burgo, S. Donato, B. Kilminster, S. Leontsinis, V.M. Mikuni, I. Neutelings, G. Rauco, P. Robmann, D. Salerno, K. Schweiger, C. Seitz, Y. Takahashi, S. Wertz, A. Zucchetta

\section{National Central University, Chung-Li, Taiwan}

T.H. Doan, C.M. Kuo, W. Lin, S.X. Liu, S.S. Yu 
National Taiwan University (NTU), Taipei, Taiwan

P. Chang, Y. Chao, K.F. Chen, P.H. Chen, W.-S. Hou, Y.y. Li, R.-S. Lu, E. Paganis, A. Psallidas, A. Steen

Chulalongkorn University, Faculty of Science, Department of Physics, Bangkok, Thailand

B. Asavapibhop, N. Srimanobhas, N. Suwonjandee

Çukurova University, Physics Department, Science and Art Faculty, Adana, Turkey

M.N. Bakirci ${ }^{50}$, A. Bat, F. Boran, S. Cerci $^{51}$, S. Damarseckin ${ }^{52}$, Z.S. Demiroglu, F. Dolek, C. Dozen, I. Dumanoglu, E. Eskut, G. Gokbulut, Y. Guler, I. Hos ${ }^{53}$, C. Isik, E.E. Kangal ${ }^{54}$, O. Kara, A. Kayis Topaksu, U. Kiminsu, M. Oglakci, G. Onengut, K. Ozdemir ${ }^{55}$, S. Ozturk ${ }^{50}$, A.E. Simsek, U.G. Tok, S. Turkcapar, I.S. Zorbakir, C. Zorbilmez

Middle East Technical University, Physics Department, Ankara, Turkey B. Isildak ${ }^{56}$, G. Karapinar ${ }^{57}$, M. Yalvac

Bogazici University, Istanbul, Turkey

I.O. Atakisi, E. Gülmez, M. Kaya ${ }^{58}$, O. Kaya ${ }^{59}$, B. Kaynak, Ö. Özçelik, S. Ozkorucuklu ${ }^{60}$, S. Tekten, E.A. Yetkin ${ }^{61}$

Istanbul Technical University, Istanbul, Turkey

A. Cakir, K. Cankocak, Y. Komurcu, S. Sen ${ }^{62}$

Institute for Scintillation Materials of National Academy of Science of Ukraine, Kharkov, Ukraine

B. Grynyov

National Scientific Center, Kharkov Institute of Physics and Technology, Kharkov, Ukraine

L. Levchuk

University of Bristol, Bristol, United Kingdom

F. Ball, E. Bhal, S. Bologna, J.J. Brooke, D. Burns, E. Clement, D. Cussans, H. Flacher, J. Goldstein, G.P. Heath, H.F. Heath, L. Kreczko, S. Paramesvaran, B. Penning, T. Sakuma, S. Seif El Nasr-Storey, D. Smith, V.J. Smith, J. Taylor, A. Titterton

Rutherford Appleton Laboratory, Didcot, United Kingdom

K.W. Bell, A. Belyaev ${ }^{63}$, C. Brew, R.M. Brown, D. Cieri, D.J.A. Cockerill, J.A. Coughlan, K. Harder, S. Harper, J. Linacre, K. Manolopoulos, D.M. Newbold, E. Olaiya, D. Petyt, T. Reis, T. Schuh, C.H. Shepherd-Themistocleous, A. Thea, I.R. Tomalin, T. Williams, W.J. Womersley

\section{Imperial College, London, United Kingdom}

R. Bainbridge, P. Bloch, J. Borg, S. Breeze, O. Buchmuller, A. Bundock, GurpreetSingh CHAHAL ${ }^{64}$, D. Colling, P. Dauncey, G. Davies, M. Della Negra, R. Di Maria, P. Everaerts, G. Hall, G. Iles, T. James, M. Komm, C. Laner, L. Lyons, A.-M. Magnan, 
S. Malik, A. Martelli, V. Milosevic, J. Nash ${ }^{65}$, V. Palladino, M. Pesaresi, D.M. Raymond, A. Richards, A. Rose, E. Scott, C. Seez, A. Shtipliyski, M. Stoye, T. Strebler, S. Summers, A. Tapper, K. Uchida, T. Virdee ${ }^{16}$, N. Wardle, D. Winterbottom, J. Wright, A.G. Zecchinelli, S.C. Zenz

Brunel University, Uxbridge, United Kingdom

J.E. Cole, P.R. Hobson, A. Khan, P. Kyberd, C.K. Mackay, A. Morton, I.D. Reid, L. Teodorescu, S. Zahid

Baylor University, Waco, U.S.A.

K. Call, J. Dittmann, K. Hatakeyama, C. Madrid, B. McMaster, N. Pastika, C. Smith

Catholic University of America, Washington, DC, U.S.A.

R. Bartek, A. Dominguez, R. Uniyal

The University of Alabama, Tuscaloosa, U.S.A.

A. Buccilli, S.I. Cooper, C. Henderson, P. Rumerio, C. West

Boston University, Boston, U.S.A.

D. Arcaro, T. Bose, Z. Demiragli, D. Gastler, S. Girgis, D. Pinna, C. Richardson, J. Rohlf,

D. Sperka, I. Suarez, L. Sulak, D. Zou

Brown University, Providence, U.S.A.

G. Benelli, B. Burkle, X. Coubez, D. Cutts, Y.t. Duh, M. Hadley, J. Hakala, U. Heintz, J.M. Hogan ${ }^{66}$, K.H.M. Kwok, E. Laird, G. Landsberg, J. Lee, Z. Mao, M. Narain, S. Sagir ${ }^{67}$, R. Syarif, E. Usai, D. Yu

University of California, Davis, Davis, U.S.A.

R. Band, C. Brainerd, R. Breedon, M. Calderon De La Barca Sanchez, M. Chertok, J. Conway, R. Conway, P.T. Cox, R. Erbacher, C. Flores, G. Funk, F. Jensen, W. Ko, O. Kukral, R. Lander, M. Mulhearn, D. Pellett, J. Pilot, M. Shi, D. Stolp, D. Taylor, K. Tos, M. Tripathi, Z. Wang, F. Zhang

University of California, Los Angeles, U.S.A.

M. Bachtis, C. Bravo, R. Cousins, A. Dasgupta, A. Florent, J. Hauser, M. Ignatenko, N. Mccoll, W.A. Nash, S. Regnard, D. Saltzberg, C. Schnaible, B. Stone, V. Valuev

University of California, Riverside, Riverside, U.S.A.

K. Burt, R. Clare, J.W. Gary, S.M.A. Ghiasi Shirazi, G. Hanson, G. Karapostoli, E. Kennedy, O.R. Long, M. Olmedo Negrete, M.I. Paneva, W. Si, L. Wang, H. Wei, S. Wimpenny, B.R. Yates, Y. Zhang

University of California, San Diego, La Jolla, U.S.A.

J.G. Branson, P. Chang, S. Cittolin, M. Derdzinski, R. Gerosa, D. Gilbert, B. Hashemi, D. Klein, V. Krutelyov, J. Letts, M. Masciovecchio, S. May, S. Padhi, M. Pieri, V. Sharma, M. Tadel, F. Würthwein, A. Yagil, G. Zevi Della Porta 
University of California, Santa Barbara - Department of Physics, Santa Barbara, U.S.A.

N. Amin, R. Bhandari, C. Campagnari, M. Citron, V. Dutta, M. Franco Sevilla, L. Gouskos, J. Incandela, B. Marsh, H. Mei, A. Ovcharova, H. Qu, J. Richman, U. Sarica, D. Stuart, S. Wang, J. Yoo

\section{California Institute of Technology, Pasadena, U.S.A.}

D. Anderson, A. Bornheim, J.M. Lawhorn, N. Lu, H.B. Newman, T.Q. Nguyen, J. Pata, M. Spiropulu, J.R. Vlimant, S. Xie, Z. Zhang, R.Y. Zhu

Carnegie Mellon University, Pittsburgh, U.S.A.

M.B. Andrews, T. Ferguson, T. Mudholkar, M. Paulini, M. Sun, I. Vorobiev, M. Weinberg

University of Colorado Boulder, Boulder, U.S.A.

J.P. Cumalat, W.T. Ford, A. Johnson, E. MacDonald, T. Mulholland, R. Patel, A. Perloff, K. Stenson, K.A. Ulmer, S.R. Wagner

Cornell University, Ithaca, U.S.A.

J. Alexander, J. Chaves, Y. Cheng, J. Chu, A. Datta, A. Frankenthal, K. Mcdermott, N. Mirman, J.R. Patterson, D. Quach, A. Rinkevicius ${ }^{68}$, A. Ryd, S.M. Tan, Z. Tao, J. Thom, P. Wittich, M. Zientek

Fermi National Accelerator Laboratory, Batavia, U.S.A.

S. Abdullin, M. Albrow, M. Alyari, G. Apollinari, A. Apresyan, A. Apyan, S. Banerjee, L.A.T. Bauerdick, A. Beretvas, J. Berryhill, P.C. Bhat, K. Burkett, J.N. Butler, A. Canepa, G.B. Cerati, H.W.K. Cheung, F. Chlebana, M. Cremonesi, J. Duarte, V.D. Elvira, J. Freeman, Z. Gecse, E. Gottschalk, L. Gray, D. Green, S. Grünendahl, O. Gutsche, AllisonReinsvold Hall, J. Hanlon, R.M. Harris, S. Hasegawa, R. Heller, J. Hirschauer, B. Jayatilaka, S. Jindariani, M. Johnson, U. Joshi, B. Klima, M.J. Kortelainen, B. Kreis, S. Lammel, J. Lewis, D. Lincoln, R. Lipton, M. Liu, T. Liu, J. Lykken, K. Maeshima, J.M. Marraffino, D. Mason, P. McBride, P. Merkel, S. Mrenna, S. Nahn, V. O'Dell, V. Papadimitriou, K. Pedro, C. Pena, G. Rakness, F. Ravera, L. Ristori, B. Schneider, E. Sexton-Kennedy, N. Smith, A. Soha, W.J. Spalding, L. Spiegel, S. Stoynev, J. Strait, N. Strobbe, L. Taylor, S. Tkaczyk, N.V. Tran, L. Uplegger, E.W. Vaandering, C. Vernieri, M. Verzocchi, R. Vidal, M. Wang, H.A. Weber

University of Florida, Gainesville, U.S.A.

D. Acosta, P. Avery, P. Bortignon, D. Bourilkov, A. Brinkerhoff, L. Cadamuro, A. Carnes,

V. Cherepanov, D. Curry, F. Errico, R.D. Field, S.V. Gleyzer, B.M. Joshi, M. Kim, J. Konigsberg, A. Korytov, K.H. Lo, P. Ma, K. Matchev, N. Menendez, G. Mitselmakher, D. Rosenzweig, K. Shi, J. Wang, S. Wang, X. Zuo

Florida International University, Miami, U.S.A.

Y.R. Joshi

Florida State University, Tallahassee, U.S.A.

T. Adams, A. Askew, S. Hagopian, V. Hagopian, K.F. Johnson, R. Khurana, T. Kolberg, G. Martinez, T. Perry, H. Prosper, C. Schiber, R. Yohay, J. Zhang 
Florida Institute of Technology, Melbourne, U.S.A.

M.M. Baarmand, V. Bhopatkar, M. Hohlmann, D. Noonan, M. Rahmani, M. Saunders, F. Yumiceva

University of Illinois at Chicago (UIC), Chicago, U.S.A.

M.R. Adams, L. Apanasevich, D. Berry, R.R. Betts, R. Cavanaugh, X. Chen, S. Dittmer, O. Evdokimov, C.E. Gerber, D.A. Hangal, D.J. Hofman, K. Jung, C. Mills, T. Roy, M.B. Tonjes, N. Varelas, H. Wang, X. Wang, Z. Wu

The University of Iowa, Iowa City, U.S.A.

M. Alhusseini, B. Bilki ${ }^{69}$, W. Clarida, K. Dilsiz ${ }^{70}$, S. Durgut, R.P. Gandrajula, M. Haytmyradov, V. Khristenko, O.K. Köseyan, J.-P. Merlo, A. Mestvirishvili ${ }^{71}$, A. Moeller, J. Nachtman, H. Ogul ${ }^{72}$, Y. Onel, F. Ozok ${ }^{73}$, A. Penzo, C. Snyder, E. Tiras, J. Wetzel

Johns Hopkins University, Baltimore, U.S.A.

B. Blumenfeld, A. Cocoros, N. Eminizer, D. Fehling, L. Feng, A.V. Gritsan, W.T. Hung, P. Maksimovic, J. Roskes, M. Swartz, M. Xiao

The University of Kansas, Lawrence, U.S.A.

C. Baldenegro Barrera, P. Baringer, A. Bean, S. Boren, J. Bowen, A. Bylinkin, T. Isidori, S. Khalil, J. King, G. Krintiras, A. Kropivnitskaya, C. Lindsey, D. Majumder, W. Mcbrayer, N. Minafra, M. Murray, C. Rogan, C. Royon, S. Sanders, E. Schmitz, J.D. Tapia Takaki, Q. Wang, J. Williams, G. Wilson

Kansas State University, Manhattan, U.S.A.

S. Duric, A. Ivanov, K. Kaadze, D. Kim, Y. Maravin, D.R. Mendis, T. Mitchell, A. Modak, A. Mohammadi

Lawrence Livermore National Laboratory, Livermore, U.S.A.

F. Rebassoo, D. Wright

University of Maryland, College Park, U.S.A.

A. Baden, O. Baron, A. Belloni, S.C. Eno, Y. Feng, N.J. Hadley, S. Jabeen, G.Y. Jeng, R.G. Kellogg, J. Kunkle, A.C. Mignerey, S. Nabili, F. Ricci-Tam, M. Seidel, Y.H. Shin, A. Skuja, S.C. Tonwar, K. Wong

Massachusetts Institute of Technology, Cambridge, U.S.A.

D. Abercrombie, B. Allen, A. Baty, R. Bi, S. Brandt, W. Busza, I.A. Cali, M. D'Alfonso, G. Gomez Ceballos, M. Goncharov, P. Harris, D. Hsu, M. Hu, M. Klute, D. Kovalskyi, Y.-J. Lee, P.D. Luckey, B. Maier, A.C. Marini, C. Mcginn, C. Mironov, S. Narayanan, X. Niu, C. Paus, D. Rankin, C. Roland, G. Roland, Z. Shi, G.S.F. Stephans, K. Sumorok, K. Tatar, D. Velicanu, J. Wang, T.W. Wang, B. Wyslouch

University of Minnesota, Minneapolis, U.S.A.

A.C. Benvenuti ${ }^{\dagger}$, R.M. Chatterjee, A. Evans, S. Guts, P. Hansen, J. Hiltbrand, Sh. Jain, S. Kalafut, Y. Kubota, Z. Lesko, J. Mans, R. Rusack, M.A. Wadud

University of Mississippi, Oxford, U.S.A.

J.G. Acosta, S. Oliveros 
University of Nebraska-Lincoln, Lincoln, U.S.A.

K. Bloom, D.R. Claes, C. Fangmeier, L. Finco, F. Golf, R. Gonzalez Suarez, R. Kamalieddin, I. Kravchenko, J.E. Siado, G.R. Snow, B. Stieger

State University of New York at Buffalo, Buffalo, U.S.A.

C. Harrington, I. Iashvili, A. Kharchilava, C. Mclean, D. Nguyen, A. Parker, J. Pekkanen,

S. Rappoccio, B. Roozbahani

Northeastern University, Boston, U.S.A.

G. Alverson, E. Barberis, C. Freer, Y. Haddad, A. Hortiangtham, G. Madigan, D.M. Morse,

T. Orimoto, L. Skinnari, A. Tishelman-Charny, T. Wamorkar, B. Wang, A. Wisecarver,

D. Wood

Northwestern University, Evanston, U.S.A.

S. Bhattacharya, J. Bueghly, T. Gunter, K.A. Hahn, N. Odell, M.H. Schmitt, K. Sung, M. Trovato, M. Velasco

University of Notre Dame, Notre Dame, U.S.A.

R. Bucci, N. Dev, R. Goldouzian, M. Hildreth, K. Hurtado Anampa, C. Jessop, D.J. Karmgard, K. Lannon, W. Li, N. Loukas, N. Marinelli, I. Mcalister, F. Meng, C. Mueller, Y. Musienko ${ }^{35}$, M. Planer, R. Ruchti, P. Siddireddy, G. Smith, S. Taroni, M. Wayne, A. Wightman, M. Wolf, A. Woodard

The Ohio State University, Columbus, U.S.A.

J. Alimena, B. Bylsma, L.S. Durkin, S. Flowers, B. Francis, C. Hill, W. Ji, A. Lefeld, T.Y. Ling, B.L. Winer

\section{Princeton University, Princeton, U.S.A.}

S. Cooperstein, G. Dezoort, P. Elmer, J. Hardenbrook, N. Haubrich, S. Higginbotham, A. Kalogeropoulos, S. Kwan, D. Lange, M.T. Lucchini, J. Luo, D. Marlow, K. Mei, I. Ojalvo, J. Olsen, C. Palmer, P. Piroué, J. Salfeld-Nebgen, D. Stickland, C. Tully, Z. Wang

University of Puerto Rico, Mayaguez, U.S.A.

S. Malik, S. Norberg

Purdue University, West Lafayette, U.S.A.

A. Barker, V.E. Barnes, S. Das, L. Gutay, M. Jones, A.W. Jung, A. Khatiwada, B. Mahakud, D.H. Miller, G. Negro, N. Neumeister, C.C. Peng, S. Piperov, H. Qiu, J.F. Schulte, J. Sun, F. Wang, R. Xiao, W. Xie

Purdue University Northwest, Hammond, U.S.A.

T. Cheng, J. Dolen, N. Parashar

Rice University, Houston, U.S.A.

K.M. Ecklund, S. Freed, F.J.M. Geurts, M. Kilpatrick, Arun Kumar, W. Li, B.P. Padley, R. Redjimi, J. Roberts, J. Rorie, W. Shi, A.G. Stahl Leiton, Z. Tu, A. Zhang 
University of Rochester, Rochester, U.S.A.

A. Bodek, P. de Barbaro, R. Demina, J.L. Dulemba, C. Fallon, T. Ferbel, M. Galanti, A. Garcia-Bellido, J. Han, O. Hindrichs, A. Khukhunaishvili, E. Ranken, P. Tan, R. Taus

Rutgers, The State University of New Jersey, Piscataway, U.S.A.

B. Chiarito, J.P. Chou, A. Gandrakota, Y. Gershtein, E. Halkiadakis, A. Hart, M. Heindl, E. Hughes, S. Kaplan, S. Kyriacou, I. Laflotte, A. Lath, R. Montalvo, K. Nash, M. Osherson, H. Saka, S. Salur, S. Schnetzer, D. Sheffield, S. Somalwar, R. Stone, S. Thomas, P. Thomassen

University of Tennessee, Knoxville, U.S.A.

H. Acharya, A.G. Delannoy, J. Heideman, G. Riley, S. Spanier

Texas A\&M University, College Station, U.S.A.

O. Bouhali ${ }^{74}$, A. Celik, M. Dalchenko, M. De Mattia, A. Delgado, S. Dildick, R. Eusebi,

J. Gilmore, T. Huang, T. Kamon ${ }^{75}$, S. Luo, D. Marley, R. Mueller, D. Overton, L. Perniè,

D. Rathjens, A. Safonov

Texas Tech University, Lubbock, U.S.A.

N. Akchurin, J. Damgov, F. De Guio, S. Kunori, K. Lamichhane, S.W. Lee, T. Mengke, S. Muthumuni, T. Peltola, S. Undleeb, I. Volobouev, Z. Wang, A. Whitbeck

Vanderbilt University, Nashville, U.S.A.

S. Greene, A. Gurrola, R. Janjam, W. Johns, C. Maguire, A. Melo, H. Ni, K. Padeken, F. Romeo, P. Sheldon, S. Tuo, J. Velkovska, M. Verweij

University of Virginia, Charlottesville, U.S.A.

M.W. Arenton, P. Barria, B. Cox, G. Cummings, R. Hirosky, M. Joyce, A. Ledovskoy, C. Neu, B. Tannenwald, Y. Wang, E. Wolfe, F. Xia

Wayne State University, Detroit, U.S.A.

R. Harr, P.E. Karchin, N. Poudyal, J. Sturdy, P. Thapa, S. Zaleski

University of Wisconsin - Madison, Madison, WI, U.S.A.

J. Buchanan, C. Caillol, D. Carlsmith, S. Dasu, I. De Bruyn, L. Dodd, F. Fiori, C. Galloni, B. Gomber ${ }^{76}$, M. Herndon, A. Hervé, U. Hussain, P. Klabbers, A. Lanaro, A. Loeliger, K. Long, R. Loveless, J. Madhusudanan Sreekala, T. Ruggles, A. Savin, V. Sharma, W.H. Smith, D. Teague, S. Trembath-reichert, N. Woods
$\dagger$ : Deceased
1: Also at Vienna University of Technology, Vienna, Austria
2: Also at IRFU, CEA, Université Paris-Saclay, Gif-sur-Yvette, France
3: Also at Universidade Estadual de Campinas, Campinas, Brazil
4: Also at Federal University of Rio Grande do Sul, Porto Alegre, Brazil
5: Also at UFMS, Nova Andradina, Brazil
6: Also at Universidade Federal de Pelotas, Pelotas, Brazil
7: Also at Université Libre de Bruxelles, Bruxelles, Belgium
8: Also at University of Chinese Academy of Sciences, Beijing, China 
9: Also at Institute for Theoretical and Experimental Physics named by A.I. Alikhanov of NRC 'Kurchatov Institute', Moscow, Russia

10: Also at Joint Institute for Nuclear Research, Dubna, Russia

11: Now at British University in Egypt, Cairo, Egypt

12: Now at Cairo University, Cairo, Egypt

13: Also at Purdue University, West Lafayette, U.S.A.

14: Also at Université de Haute Alsace, Mulhouse, France

15: Also at Erzincan Binali Yildirim University, Erzincan, Turkey

16: Also at CERN, European Organization for Nuclear Research, Geneva, Switzerland

17: Also at RWTH Aachen University, III. Physikalisches Institut A, Aachen, Germany

18: Also at University of Hamburg, Hamburg, Germany

19: Also at Brandenburg University of Technology, Cottbus, Germany

20: Also at Institute of Physics, University of Debrecen, Debrecen, Hungary, Debrecen, Hungary

21: Also at Institute of Nuclear Research ATOMKI, Debrecen, Hungary

22: Also at MTA-ELTE Lendület CMS Particle and Nuclear Physics Group, Eötvös Loránd University, Budapest, Hungary, Budapest, Hungary

23: Also at IIT Bhubaneswar, Bhubaneswar, India, Bhubaneswar, India

24: Also at Institute of Physics, Bhubaneswar, India

25: Also at Shoolini University, Solan, India

26: Also at University of Visva-Bharati, Santiniketan, India

27: Also at Isfahan University of Technology, Isfahan, Iran

28: Also at Italian National Agency for New Technologies, Energy and Sustainable Economic Development, Bologna, Italy

29: Also at Centro Siciliano di Fisica Nucleare e di Struttura Della Materia, Catania, Italy

30: Also at Scuola Normale e Sezione dell'INFN, Pisa, Italy

31: Also at Riga Technical University, Riga, Latvia, Riga, Latvia

32: Also at Malaysian Nuclear Agency, MOSTI, Kajang, Malaysia

33: Also at Consejo Nacional de Ciencia y Tecnología, Mexico City, Mexico

34: Also at Warsaw University of Technology, Institute of Electronic Systems, Warsaw, Poland

35: Also at Institute for Nuclear Research, Moscow, Russia

36: Now at National Research Nuclear University 'Moscow Engineering Physics Institute' (MEPhI), Moscow, Russia

37: Also at Institute of Nuclear Physics of the Uzbekistan Academy of Sciences, Tashkent, Uzbekistan

38: Also at St. Petersburg State Polytechnical University, St. Petersburg, Russia

39: Also at University of Florida, Gainesville, U.S.A.

40: Also at Imperial College, London, United Kingdom

41: Also at P.N. Lebedev Physical Institute, Moscow, Russia

42: Also at California Institute of Technology, Pasadena, U.S.A.

43: Also at Budker Institute of Nuclear Physics, Novosibirsk, Russia

44: Also at Faculty of Physics, University of Belgrade, Belgrade, Serbia

45: Also at Università degli Studi di Siena, Siena, Italy

46: Also at INFN Sezione di Pavia ${ }^{a}$, Università di Pavia ${ }^{b}$, Pavia, Italy, Pavia, Italy

47: Also at National and Kapodistrian University of Athens, Athens, Greece

48: Also at Universität Zürich, Zurich, Switzerland

49: Also at Stefan Meyer Institute for Subatomic Physics, Vienna, Austria, Vienna, Austria

50: Also at Gaziosmanpasa University, Tokat, Turkey

51: Also at Adiyaman University, Adiyaman, Turkey 
52: Also at Şırnak University, Sirnak, Turkey

53: Also at Istanbul Aydin University, Istanbul, Turkey

54: Also at Mersin University, Mersin, Turkey

55: Also at Piri Reis University, Istanbul, Turkey

56: Also at Ozyegin University, Istanbul, Turkey

57: Also at Izmir Institute of Technology, Izmir, Turkey

58: Also at Marmara University, Istanbul, Turkey

59: Also at Kafkas University, Kars, Turkey

60: Also at Istanbul University, Istanbul, Turkey

61: Also at Istanbul Bilgi University, Istanbul, Turkey

62: Also at Hacettepe University, Ankara, Turkey

63: Also at School of Physics and Astronomy, University of Southampton, Southampton, United Kingdom

64: Also at IPPP Durham University, Durham, United Kingdom

65: Also at Monash University, Faculty of Science, Clayton, Australia

66: Also at Bethel University, St. Paul, Minneapolis, U.S.A., St. Paul, U.S.A.

67: Also at Karamanoğlu Mehmetbey University, Karaman, Turkey

68: Also at Vilnius University, Vilnius, Lithuania

69: Also at Beykent University, Istanbul, Turkey, Istanbul, Turkey

70: Also at Bingol University, Bingol, Turkey

71: Also at Georgian Technical University, Tbilisi, Georgia

72: Also at Sinop University, Sinop, Turkey

73: Also at Mimar Sinan University, Istanbul, Istanbul, Turkey

74: Also at Texas A\&M University at Qatar, Doha, Qatar

75: Also at Kyungpook National University, Daegu, Korea, Daegu, Korea

76: Also at University of Hyderabad, Hyderabad, India 